\title{
THE THEOREM OF THE COMPLEMENT FOR NESTED SUBPFAFFIAN SETS
}

\author{
J.-M. LION AND P. SPEISSEGGER
}

\begin{abstract}
Let $\mathcal{R}$ be an o-minimal expansion of the real field, and let $\mathcal{L}_{\text {nest }}(\mathcal{R})$ be the language consisting of all nested Rolle leaves over $\mathcal{R}$. We call a set nested subpfaffian over $\mathcal{R}$ if it is the projection of a positive boolean combination of definable sets and nested Rolle leaves over $\mathcal{R}$. Assuming that $\mathcal{R}$ admits analytic cell decomposition, we prove that the complement of a nested subpfaffian set over $\mathcal{R}$ is again a nested subpfaffian set over $\mathcal{R}$. As a corollary, we obtain that if $\mathcal{R}$ admits analytic cell decomposition, then the pfaffian closure $\mathcal{P}(\mathcal{R})$ of $\mathcal{R}$ is obtained by adding to $\mathcal{R}$ all nested Rolle leaves over $\mathcal{R}$, a one-stage process, and that $\mathcal{P}(\mathcal{R})$ is model complete in the language $\mathcal{L}_{\text {nest }}(\mathcal{R})$.
\end{abstract}

\section{INTRODUCTION}

The basic objects we study in this paper are nested pfaffian sets over a given o-minimal expansion of the real field. Before defining them, let us briefly recall some of the history around the notion of pfaffian functions: roughly speaking, pfaffian functions are maximal solutions of triangular systems of partial differential equations with polynomial coefficients, see Khovanskii [11], Gabrielov [7] and Wilkie [21]. In his thesis [11], Khovanskii proves (among other things) that any set defined by finitely many equations and inequalities between pfaffian functions has a finite number of connected components. In the early 1980s, Van den Dries conjectured that the expansion of the real field by all pfaffian functions was model complete, which, together with Khovanskii's theorem, would imply that this expansion is o-minimal. (For generalities on o-minimal structures, we refer the reader to Van den Dries and Miller [5].)

Wilkie [22] used a different approach to obtain the first general o-minimality result for pfaffian functions, showing that the real field expanded by all totally defined pfaffian functions is o-minimal. Based on Lion and Rolin [13], this theorem was strengthened in the following way: given an o-minimal expansion

Date: September 3, 2018.

1991 Mathematics Subject Classification. Primary 14P10, 58A17; Secondary 03 C99.

Key words and phrases. O-minimal structures, pfaffian systems, analytic stratification.

Supported by the CNRS of France and NSERC of Canada grant RGPIN 261961. We thank the Newton Institute at Cambridge and the Fields Institute in Toronto, where parts of this paper were written. 
$\mathcal{R}$ of the real field, we call a function pfaffian over $\mathcal{R}$ if it is a maximal solution of a triangular system of partial differential equations with coefficients definable in $\mathcal{R}$. Then [20] there is an o-minimal expansion $\mathcal{P}(\mathcal{R})$ of $\mathcal{R}$, called the pfaffian closure of $\mathcal{R}$, such that every pfaffian function over $\mathcal{P}(\mathcal{R})$ is definable in $\mathcal{P}(\mathcal{R})$.

However, none of the above o-minimality proofs establish the model completeness of the respective structures in any meaningfull language, and Gabrielov restated the question in [8. Based on techniques used in [15], we give here a natural language $\mathcal{L}_{\text {nest }}(\mathcal{R})$ in which $\mathcal{P}(\mathcal{R})$ is model complete, in the case where $\mathcal{R}$ admits analytic cell decomposition. To do this, we draw inspiration from the setting in [11], where pfaffian functions are replaced by nested separating integral manifolds. We need a few definitions to state the precise theorem.

We denote by $G_{n}^{l}$ the Grassmannian of all $l$-dimensional linear subspaces of $\mathbb{R}^{n}$. Let $M \subseteq \mathbb{R}^{n}$ be a $C^{1}$-submanifold of dimension $m$. Throughout this paper, we identify the tangent space $T_{x} M$ of $M$ at $x \in M$ with an element of $G_{n}^{m}$ in the usual way, and we define an l-distribution on $M$ to be a map $d: M \longrightarrow G_{n}^{l}$ such that $d(x) \subseteq T_{x} M$ for all $x \in M$. For example, the $m$-distribution $g_{M}$ on $M$ defined by $g_{M}(x):=T_{x} M$ is the Gauss map of $M$.

Let $d$ be an $l$-distribution on $M$. An immersed manifold $V \subseteq M$ of dimension $l$ is called an integral manifold of $d$ if $T_{x} V=d(x)$ for all $x \in V$. A leaf of $d$ is a maximal connected integral manifold of $d$.

Assume now that $l=m-1$. Then a leaf $V$ of $d$ is Rolle (see Moussu and Roche [18]) if $V$ is a closed submanifold of $M$ and for every $C^{1}$-curve $\gamma:[0,1] \longrightarrow M$ such that $\gamma(0), \gamma(1) \in V$, there exists a $t \in[0,1]$ such that $\gamma^{\prime}(t) \in d(\gamma(t))$. The following criterion for the Rolle property is crucial to our paper:

Haefliger's Theorem [10, 19]. Assume that $M$ and $d$ as above are analytic and that $M$ is simply connected and $d$ is integrable (Definition 2.3). Then every leaf of $d$ is a Rolle leaf.

Let $d=\left(d_{0}, \ldots, d_{k}\right)$ be a tuple of distributions on $M$. We call $d$ nested if each $d_{j}$ is an $(m-j)$-distribution on $M$ and $d_{k}(x) \subseteq d_{k-1}(x) \subseteq \cdots \subseteq d_{0}(x)=$ $g_{M}(x)$ for all $x \in M$.

Assume that $d$ is nested, and let $V=\left(V_{0}, \ldots, V_{k}\right)$ be a tuple of immersed manifolds contained in $M$. We call $V$ a nested integral manifold of $d$ if each $V_{j}$ is an integral manifold of $d_{j}$ and $V_{0} \supseteq \cdots \supseteq V_{k}$. Moreover, $V$ is a nested leaf (respectively, nested Rolle leaf) of $d$, if $V_{0}=M$ and for $j=1, \ldots, k$, the set $V_{j}$ is a leaf (respectively, Rolle leaf) of the restriction $d_{j} \uparrow_{V_{j-1}}$ of $d_{j}$ to $V_{j-1}$. Note that in this situation, $d_{j} \uparrow_{V_{j-1}}$ is of class $C^{1}$ and $d_{j}(x) \subseteq T_{x} V_{j-1}$ is of codimension 1 for all $x \in V_{j-1}$; in particular, $\operatorname{dim} V_{j}=m-j$. 
Example 1 [20]. Let $\Omega=\left(\omega_{1}, \ldots, \omega_{k}\right)$ be a family of differential 1-forms on $M$, and assume that $\Omega$ is nonsingular, that is, $\omega_{1}(x) \wedge \cdots \wedge \omega_{k}(x) \neq 0$ for all $x \in M$. For $j=1, \ldots, k$, put $d_{j}(x):=\operatorname{ker} \omega_{1}(x) \cap \cdots \cap \operatorname{ker} \omega_{j}(x)$; then $d:=\left(g_{M}, d_{1}, \ldots, d_{k}\right)$ is a nested distribution on $M$.

Conversely, let $d=\left(d_{0}, \ldots, d_{k}\right)$ be a nested distribution on $M$ and assume that $M$ is simply connected. Define unit vector fields $a_{j}=\left(a_{j 1}, \ldots, a_{j n}\right)$ on $M$, for $j=1, \ldots, k$, by induction on $j$ as follows: let $a_{1}$ be one of the two unit vector fields orthogonal to $d_{1}$, and for $j>1$ let $a_{j}$ be one of the two unit vector fields orthogonal to the vector space field spanned by $d_{j} \cup\left\{a_{1}, \ldots, a_{j-1}\right\}$. Finally, put $\omega_{j}:=a_{j 1} d x_{1}+\cdots+a_{j n} d x_{n}$ for $j=1, \ldots, k$. Then $\Omega:=\left(\omega_{1}, \ldots, \omega_{k}\right)$ is a nonsingular family of differential 1-forms on $M$.

In the notation used before Example 1, if $M$ is simply connected, then $V$ is a nested integral manifold (leaf, Rolle leaf) of this $d$ if and only if $V_{1}$ is an integral manifold (leaf, Rolle leaf) of $\omega_{1}$-as defined in [20] - and for $j=2, \ldots, k, V_{j}$ is an integral manifold (leaf, Rolle leaf) of the pull-back of $\omega_{j}$ on $V_{j-1}$.

Let $\mathcal{R}$ be an o-minimal expansion of the real field, and assume that $M$ is definable in $\mathcal{R}$ and of class $C^{2}$ (the reason for the latter assumption is to be consistent with pull-backs, see the conventions below). Let $d=\left(d_{0}, \ldots, d_{k}\right)$ be a nested distribution on $M$ and $V=\left(V_{0}, \ldots, V_{k}\right)$ be a nested integral manifold of $d$. For $l \leq n$ we identify $G_{n}^{l}$ with an algebraic (and hence definable in $\mathcal{R}$ ) subvariety of $\mathbb{R}^{n^{2}}$ (see the conventions below for details). We call $d$ definable if $d$ is a definable map under this identification. If $d$ is definable, we call $V$ a nested integral manifold over $\mathcal{R}$, and if $V$ is a nested Rolle leaf of $d$, we call $V$ a nested Rolle leaf over $\mathcal{R}$. Note that in the latter situation, the leaves $V_{0}, \ldots, V_{k}$ are uniquely determined by $d$ and $V_{k}$, but that $V_{k}$ is not definable in $\mathcal{R}$ in general. For convenience, we call a set $W \subseteq \mathbb{R}^{n}$ an integral manifold over $\mathcal{R}$ if there is a nested integral manifold $\left(W_{0}, \ldots, W_{k}\right)$ over $\mathcal{R}$ with $W_{k}=W$, and if in addition $\left(W_{0}, \ldots, W_{k}\right)$ is a nested Rolle leaf over $\mathcal{R}$, we call $W$ a Rolle leaf over $\mathcal{R}$.

Example 2. Let $C \subseteq \mathbb{R}^{n}$ be a definable $C^{2}$-cell. Taking $M=C$ and $d$ to be the Gauss map on $M$ makes $C$ trivially into a Rolle leaf over $\mathcal{R}$.

A set $X \subseteq \mathbb{R}^{n}$ is a basic nested pfaffian set over $\mathcal{R}$, if there are a definable set $A \subseteq \mathbb{R}^{n}$ and a Rolle leaf $W \subseteq \mathbb{R}^{n}$ over $\mathcal{R}$ such that $X=A \cap W$. A nested pfaffian set over $\mathcal{R}$ is a finite union of basic nested pfaffian sets over $\mathcal{R}$, and a nested subpfaffian set over $\mathcal{R}$ is the image under a coordinate projection of a nested pfaffian set over $\mathcal{R}$.

We let $\mathcal{L}_{\text {nest }}(\mathcal{R})$ be the collection of all Rolle leaves over $\mathcal{R}$, and we denote by $\mathcal{N}(\mathcal{R})$ the expansion of $\mathcal{R}$ by all $W \in \mathcal{L}_{\text {nest }}(\mathcal{R})$. It follows from $C^{2}$-cell decomposition in $\mathcal{R}$ and Example 2 that every set definable in $\mathcal{R}$ is quantifierfree definable in $\mathcal{N}(\mathcal{R})$. 
Khovanskii theory as in [18, 20] generalizes in a straightforward way to the setting of Rolle leaves over $\mathcal{R}$ (Sections 2 and 3 ). It follows in particular that every nested subpfaffian set over $\mathcal{R}$ has finitely many connected components, and every Rolle leaf over $\mathcal{R}$ is definable in $\mathcal{P}(\mathcal{R})$ (Proposition 3.6). Hence, every nested subpfaffian set $X$ over $\mathcal{R}$ is definable in the o-minimal structure $\mathcal{P}(\mathcal{R})$; we denote its dimension by $\operatorname{dim} X$. Building on these observations, we prove:

Main Theorem. Assume that $\mathcal{R}$ admits analytic cell decomposition. Then the complement of every nested subpfaffian set over $\mathcal{R}$ is again nested subpfaffian over $\mathcal{R}$; in particular, $\mathcal{N}(\mathcal{R})$ is model complete.

The Main Theorem implies that, in the construction of $\mathcal{P}(\mathcal{R})$ in [20], every Rolle leaf added to $\mathcal{R}$ is a nested subpfaffian set over $\mathcal{R}$ (Proposition 11.1).

Corollary 1. If $\mathcal{R}$ admits analytic cell decomposition, then $\mathcal{N}(\mathcal{R})$ is interdefinable with $\mathcal{P}(\mathcal{R})$; in particular, $\mathcal{P}(\mathcal{R})$ is model complete in $\mathcal{L}_{\text {nest }}(\mathcal{R})$.

The model completeness of $\mathcal{P}(\mathcal{R})$ in the language $\mathcal{L}_{\text {nest }}(\mathcal{R})$ remains an open problem if $\mathcal{R}$ does not admit analytic cell decomposition. Also, even in the analytic case, we do not know whether the reduct of $\mathcal{P}(\mathcal{R})$ generated by all pfaffian functions over $\mathcal{R}$ is model complete.

To prove the Main Theorem, we use Corollary 2.9 of [6], with $\Lambda$ there equal to the collection of all nested pfaffian sets over $\mathcal{R}$ contained in $[-1,1]^{n}$, for $n \in \mathbb{N}$. This means that it suffices to establish Axioms (I)-(IV) there; they follow easily from the following list of statements, which correspond to theorems proved in this paper:

(P1) every set definable in $\mathcal{R}$ is nested pfaffian over $\mathcal{R}$;

(P2) the union, the intersection and the cartesian product of two nested pfaffian sets over $\mathcal{R}$ are nested pfaffian over $\mathcal{R}$, and each connected component of a nested pfaffian set over $\mathcal{R}$ is nested pfaffian over $\mathcal{R}$;

(P3) if $X \subseteq \mathbb{R}^{n}$ is nested pfaffian over $\mathcal{R}$ and $1 \leq m \leq n$, there is a finite collection $\mathcal{P}$ of analytic manifolds contained in $X$ such that $\Pi_{m}(X)=$ $\bigcup_{Y \in \mathcal{P}} \Pi_{m}(Y)$ and for each $Y \in \mathcal{P}$, the set $Y$ is nested pfaffian over $\mathcal{R}$, $\operatorname{dim} Y \leq m$ and there is a strictly increasing $\lambda:\{1, \ldots, \operatorname{dim} Y\} \longrightarrow$ $\{1, \ldots, m\}$ such that $\Pi_{\lambda} \uparrow_{Y}: Y \longrightarrow \mathbb{R}^{\operatorname{dim} Y}$ is an immersion;

(P4) if $X \subseteq \mathbb{R}^{n}$ is nonempty and nested pfaffian over $\mathcal{R}$, there is a closed, nested subpfaffian set $Y \subseteq \mathbb{R}^{n}$ over $\mathcal{R}$ such that fr $X \subseteq Y$ and $\operatorname{dim} Y<$ $\operatorname{dim} X$.

Here, for $l \geq m$, the map $\Pi_{m}: \mathbb{R}^{l} \longrightarrow \mathbb{R}^{m}$ denotes the projection on the first $m$ coordinates, and for every strictly increasing $\lambda:\{1, \ldots, m\} \longrightarrow$ $\{1, \ldots, l\}$, the map $\Pi_{\lambda}: \mathbb{R}^{l} \longrightarrow \mathbb{R}^{m}$ denotes the projection $\Pi_{\lambda}\left(x_{1}, \ldots, x_{l}\right):=$ 
$\left(x_{\lambda(1)}, \ldots, x_{\lambda(m)}\right)$. Also, for any set $S \subseteq \mathbb{R}^{n}$, we denote by cl $S$ the topological closure of $S$, and we define the frontier of $S$ as the set fr $S:=\operatorname{cl} S \backslash S$.

Statement (P1) follows from Example 2, and (P2) follows from Khovanskii theory for nested pfaffian sets over $\mathcal{R}$ (Corollary 3.4). Statement (P3) follows from the Fiber Cutting Lemma for nested pfaffian sets over $\mathcal{R}$ (Corollary 8.14), which is obtained using an approach similar to Gabrielov's in [9]. The main contribution of this paper is to establish (P4). To explain how this is done, we let $W \subseteq \mathbb{R}^{n}$ be a Rolle leaf over $\mathcal{R}$. By Khovanskii theory again (Corollary 3.3), statement (P4) follows from

Theorem 1. Assume that $\mathcal{R}$ admits analytic cell decomposition. Then there is a closed, nested subpfaffian set $Y \subseteq \mathbb{R}^{n}$ over $\mathcal{R}$ such that fr $W \subseteq Y$ and $\operatorname{dim} Y<\operatorname{dim} W$.

Theorem 1 was proved in the special case $\operatorname{dim} W=n-1$ by Cano et al. in [3]. For the proof of the general case (Section 10), we let $d=\left(d_{0}, \ldots, d_{k}\right)$ be a definable, nested distribution on a bounded, definable $C^{2}$-manifold $M \subseteq \mathbb{R}^{n}$ and $V=\left(V_{0}, \ldots, V_{k}\right)$ be a nested Rolle leaf of $d$ such that $W=V_{k}$. We consider fr $W$ as a Hausdorff limit of a certain sequence of integral manifolds of a definable nested distribution $d^{\prime}$ on $M$ derived from $d$ (Section 4). We then use the method of blowing up along $d^{\prime}$ (Section [5), similar to [15], to recoverroughly speaking - distributions on the frontier of $M$, such that fr $W$ is almost everywhere an integral manifold of one of these distributions. (Strictly speaking, these distributions are recovered on the frontier of the manifold obtained from $M$ by blowing up and have the described property for the corresponding lifting of $W$; to keep notations simple, we continue using $M$ and $W$ in the introduction.) The main problems solved in this paper are the following: we did not know in [15] if

(a) the distributions recovered in this way were components of definable nested distributions;

(b) the integral manifolds in question were contained in Rolle leaves over $\mathcal{R}$ of the same dimension.

Here we deal with (a) and (b) separately; we establish (a) for the case that $\mathcal{R}$ is any o-minimal expansion of the real field, but we need to assume that $\mathcal{R}$ admits analytic cell decomposition to establish (b).

For (a), we define the degree of $d$ to be the number of component distributions of $d$ whose associated foliation of $M$ is not definable in $\mathcal{R}$ (Section 2). We show in Section 4 that this degree behaves well in the following sense: the nested distribution $d^{\prime}$ derived from $d$ used to describe fr $W$ as a Hausdorff limit, as mentioned above, has degree less than or equal to that of $d$. Moreover, we also prove that the negligible set, off which fr $W$ is a finite union of integral manifolds of the recovered distributions, is a union of Hausdorff limits of the 
same type obtained from distributions of degree less than or equal to that of $d$. These observations and a refinement of the blowing-up method in [15] yield the following version of (a), combining Propositions 4.6 and 7.1 below:

Proposition 1. There is a $q \in \mathbb{N}$ and, for $p=1, \ldots, q$, there are $n_{p} \geq n$ and an integral manifold $U_{p} \subseteq \mathbb{R}^{n_{p}}$ over $\mathcal{R}$ such that fr $W \subseteq \Pi_{n}\left(U_{1}\right) \cup \cdots \cup \Pi_{n}\left(U_{q}\right)$ and for each $p$, the set $U_{p}$ is definable in $\mathcal{P}(\mathcal{R})$ and $\operatorname{dim} \Pi_{n}\left(U_{p}\right)<\operatorname{dim} W$.

We call an integral manifold $U \subseteq \mathbb{R}^{n}$ over $\mathcal{R}$ definable in $\mathcal{P}(\mathcal{R})$ if there is a nested integral manifold $Z=\left(Z_{0}, \ldots, Z_{l}\right)$ over $\mathcal{R}$ such that $U=Z_{l}$ and each $Z_{j}$ is definable in $\mathcal{P}(\mathcal{R})$. For (b) it now remains to show, for each of the integral manifolds $U_{p}$ of Proposition 1 , that $\Pi_{n}\left(U_{p}\right)$ is in turn contained in a subpfaffian set over $\mathcal{R}$ of dimension at most $\operatorname{dim} \Pi_{n}\left(U_{p}\right)$ (implied by Proposition 10.4).

To do so, we let $d=\left(d_{0}, \ldots, d_{k}\right)$ be a definable nested distribution on some manifold $M$ and $V=\left(V_{0}, \ldots, V_{k}\right)$ be a nested integral manifold of $d$ definable in $\mathcal{P}(\mathcal{R})$, and we try to reduce to a situation where, up to finite union and projection, the leaf $L_{k}$ of $d_{k}$ containing $V_{k}$ is a Rolle leaf $d$. To establish the Rolle property of $L_{k}$, we want to use Haefliger's Theorem; this is one of the reasons for our assumption that $\mathcal{R}$ admits analytic cell decomposition. Thus, if $k=1$, we can easily recover the Rolle property from Haefliger's Theorem using analytic cell decomposition of $M$. If $k>1$, however, we can only apply Haefliger's Theorem if $V_{k-1}$ is simply connected. Proceeding by induction on $k$, we may assume that $\left(V_{0}, \ldots, V_{k-1}\right)$ is a nested Rolle leaf over $\mathcal{R}$; therefore, what we need to establish is (see Corollary 10.2):

Proposition 2. Assume that $\mathcal{R}$ admits analytic cell decomposition. Then $V_{k-1}$ is a finite union of simply connected nested subpfaffian sets over $\mathcal{R}$ that are analytic manifolds.

To prove this, we introduce in Section 9 the notion of proper nested subpfaffian set over $\mathcal{R}$. These are certain projections of nested pfaffian sets $X \subseteq$ $[-1,1]^{n}$ over $\mathcal{R}$ that are restricted off some closed set $Z$; if $Z=\{0\}$, this means that for every $r>0$, the set $X \backslash(-r, r)^{n}$ is a restricted nested pfaffian set similar to Gabrielov's in [9] or [21]. Remarkably, based on the ideas in [9]adapted to our situation in Sections 8 and 9 -we obtain a cell decomposition theorem for certain proper nested subpfaffian sets over $\mathcal{R}$ (Proposition 9.3). Proposition 2 then follows from the observation that, up to an analytic inversion of the ambient space, $V_{k-1}$ is a restricted pfaffian set off $\{0\}$ (Example 9.2 and Proposition 10.1).

Unfortunately, Haefliger's Theorem is false without the analyticity assumption, even in the o-minimal context, see for instance [16, Section 3]. We prove there a weaker version of Haefliger's Theorem in the general o-minimal context, but we do not know if the proof of the Main Theorem goes through with this weaker version of Haefliger's Theorem. 
Conventions. Throughout this paper, all cells, manifolds, functions, maps, etc. are of class $C^{1}$, and manifolds are embedded, unless otherwise specified. We write $\mathbb{N}=\{0,1,2,3, \ldots\}$ for the set of all natural numbers. We sometimes abbreviate "analytic" as " $C \omega$ ", and we extend the usual linear ordering on $\mathbb{N} \cup\{\infty\}$ to $\mathbb{N} \cup\{\infty, \omega\}$ by putting $\omega>\infty$. We shall use "component" in place of "connected component" whenever the meaning is clear from context.

$\mathcal{R}$ denotes a fixed, but arbitrary, o-minimal expansion of the real field, and "definable" means "definable in $\mathcal{R}$ with parameters from $\mathbb{R}$ " unless indicated otherwise.

A box in $\mathbb{R}^{n}$ is a subset of the form $I_{1} \times \cdots \times I_{n}$, where each $I_{j}$ is a nonempty open interval in $\mathbb{R}$. For $x \in \mathbb{R}^{n}$, we put $|x|:=\sup \left\{\left|x_{1}\right|, \ldots,\left|x_{n}\right|\right\}$, and for $r>0$, we let $B(x, r):=\left\{y \in \mathbb{R}^{n}:|y-x|<r\right\}$.

For any set $S \subseteq \mathbb{R}^{n}$, we denote by $|S|$ the cardinality of $S$, by cl $S$ the topological closure of $S$ and by int $S$ the interior of $S$, and we define the boundary of $S$ as bd $S:=\operatorname{cl} S \backslash \operatorname{int} S$ and the frontier of $S$ as the set fr $S:=\operatorname{cl} S \backslash S$. A family $\mathcal{S}$ of subsets of $\mathbb{R}^{n}$ is a stratification if the members of $\mathcal{S}$ are pairwise disjoint and for all $S_{1}, S_{2} \in \mathcal{S}$, we have either $S_{1} \cap \operatorname{cl} S_{2}=\emptyset$ or $S_{1} \subseteq \operatorname{cl} S_{2}$. In this paper, we also use Whitney stratifications; their definition is more technical, and we refer the reader to Sections 1 and 4 of Miller and Van den Dries [5] for a thorough discussion in the o-minimal context.

We let $\Sigma_{n}$ be the collection of all permutations of $\{1, \ldots, n\}$. For $\sigma \in \Sigma_{n}$, we write $\sigma: \mathbb{R}^{n} \longrightarrow \mathbb{R}^{n}$ for the map defined by $\sigma\left(x_{1}, \ldots, x_{n}\right):=\left(x_{\sigma(1)}, \ldots, x_{\sigma(n)}\right)$.

For $l \geq m$, the map $\Pi_{m}^{l}: \mathbb{R}^{l} \longrightarrow \mathbb{R}^{m}$ denotes the projection on the first $m$ coordinates; and for every strictly increasing $\lambda:\{1, \ldots, m\} \longrightarrow$ $\{1, \ldots, l\}$, the map $\Pi_{\lambda}^{l}: \mathbb{R}^{l} \longrightarrow \mathbb{R}^{m}$ denotes the projection $\Pi_{\lambda}^{l}\left(x_{1}, \ldots, x_{l}\right):=$ $\left(x_{\lambda(1)}, \ldots, x_{\lambda(m)}\right)$. When $l$ is clear from context, we usually write $\Pi_{m}$ and $\Pi_{\lambda}$ in place of $\Pi_{m}^{l}$ and $\Pi_{\lambda}^{l}$, respectively.

We let $\mathcal{K}_{n}$ be the space of all compact subsets of $\mathbb{R}^{n}$ equipped with the Hausdorff metric. (We consider $\emptyset \in \mathcal{K}_{n}$ with $d(A, \emptyset)=\infty$ for all nonempty $A \in \mathcal{K}_{n}$.) Given a sequence $\left(A_{\iota}\right)_{\iota \in \mathbb{N}}$ of bounded subsets of $\mathbb{R}^{n}$, we say that $\left(A_{\iota}\right)$ converges to $C \in \mathcal{K}_{n}$ if the sequence $\left(\mathrm{cl} A_{\iota}\right)$ converges in $\mathcal{K}_{n}$ to $C$, and in this situation we write $C=\lim _{\iota} A_{\iota}$. We refer the reader to Kuratowski [12] for the classical results about $\mathcal{K}_{n}$; in particular, we shall often use without reference the fact that every bounded sequence in $\mathcal{K}_{n}$ contains a convergent subsequence.

Let $l \leq n$. We denote by $G_{n}^{l}$ the Grassmannian of all $l$-dimensional vector subspaces of $\mathbb{R}^{n}$. This $G_{n}^{l}$ is an analytic, real algebraic variety with a natural analytic embedding into the vector space $M_{n}$ of all real valued $(n \times n)$-matrices: each $l$-dimensional vector space $E$ is identified with the unique matrix $A_{E}$ (with respect to the standard basis of $\mathbb{R}^{n}$ ) corresponding to the orthogonal projection on the orthogonal complement of $E$ (see Section 3.4.2 of [1]); in 
particular, $E=\operatorname{ker}\left(A_{E}\right)$. We shall identify $M_{n}$ with $\mathbb{R}^{n^{2}}$ via the map $A=$ $\left(a_{i j}\right) \mapsto z_{A}=\left(z_{1}, \ldots, z_{n^{2}}\right)$ defined by $a_{i j}=z_{n(i-1)+j}$, and we identify $G_{n}^{l}$ with its image in $M_{n}$ under this map. Note that the sets $G_{n}^{0}, \ldots, G_{n}^{n}$ are the components of $G_{n}:=\bigcup_{p=0}^{n} G_{n}^{p}$.

Let $M \subseteq \mathbb{R}^{n}$ be a manifold of dimension $m \leq n$. For $\eta>0$, we say that $M$ is $\eta$-bounded if for every $x \in M$ there is a matrix $L=\left(l_{i j}\right) \in \mathrm{M}_{n-m, m}(\mathbb{R})$ such that $|L|:=\sup _{i, j}\left|l_{i, j}\right| \leq \eta$ and $T_{x} M=\left\{(u, L u): u \in \mathbb{R}^{m}\right\}$.

We call a map $d: M \longrightarrow G_{n}$ a distribution on $M$ if $d(x) \subseteq T_{x} M$ for all $x \in M$. Given two maps $d, e: M \longrightarrow G_{n}$, we write $d \cap e: M \longrightarrow G_{n}$ for the map defined by $(d \cap e)(x):=d(x) \cap e(x)$, and we write $d \subseteq e$ if $d(x) \subseteq e(x)$ for all $x \in M$. Note that, by linear algebra, if $M$ and $d, e: M \longrightarrow G_{n}$ are of class $C^{p}$, with $p \in \mathbb{N} \cup\{\infty, \omega\}$, then so is $d \cap e$. If $d: M \longrightarrow G_{n}$ is a map, we say that $d$ has dimension if $d(M) \subseteq G_{n}^{m}$ for some $m \leq n$; in this situation, we put $\operatorname{dim} d:=m$.

Assume that $M$ is of class $C^{2}$, let $N \subseteq \mathbb{R}^{l}$ be a $C^{2}$-manifold and $f: N \longrightarrow M$ a $C^{2}$-map, and let $g$ be a distribution on $M$. The pull-back of $g$ on $N$ by $f$ is the distribution $f^{*} g$ on $N$ defined by $f^{*} g(y):=\left(d f_{y}\right)^{-1}(g(f(y)))$, where $d f_{y}: T_{y} N \longrightarrow T_{f(y)} M$ is the linear map defined by the jacobian matrix of $f$ at $y$ and $\left(d f_{y}\right)^{-1}(S)$ denotes the inverse image of $S$ under this map for any $S \subseteq T_{f(y)} M$. If $N$ is a $C^{2}$-submanifold of $M$ and $f$ is the inclusion map, we write $g^{N}$ in place of $f^{*} g$.

\section{Preliminaries}

This section introduces some terminology and contains several basic lemmas needed later on. Below, for any map $f$ we denote by gr $f$ the graph of $f$.

Lemma 1.1. Let $p \geq 1$ be finite, and let $M \subseteq \mathbb{R}^{n}$ be a definable $C^{p}$-manifold of dimension $d$. Let also $m \leq n$, and assume that $\Pi_{m} \Upsilon_{M}$ has constant rank $\nu$. Then $M$ is the union of finitely many definable open subsets $N$ such that $\Pi_{m}(N)$ is a $C^{p}$-submanifold of $\mathbb{R}^{m}$ of dimension $\nu$.

Proof. Given a permutation $\sigma \in \Sigma_{m}$ and denoting by $\sigma: \mathbb{R}^{n} \longrightarrow \mathbb{R}^{n}$ the map defined by $\sigma(x):=\left(x_{\sigma(1)}, \ldots, x_{\sigma(m)}, x_{m+1}, \ldots, x_{n}\right)$, the set

$$
M_{\sigma}:=\left\{y \in M: \Pi_{\nu} \uparrow_{\sigma(M)} \text { is a submersion at } \sigma(y)\right\}
$$

is an open subset of $M$ and $M$ is the union of all $M_{\sigma}$ with $\sigma \in \Sigma_{m}$. Thus by replacing $M$ with each $\sigma\left(M_{\sigma}\right)$, we may assume that $\Pi_{\nu} \uparrow_{M}$ is a submersion; in particular, $U:=\Pi_{\nu}(M)$ is open. Since $M$ is definable, the hypotheses now imply that there is a $K \in \mathbb{N}$ such that for every $y \in \mathbb{R}^{\nu}$, the fiber $\Pi_{m}(M)_{y}$ has at most $K$ elements. For $k \in\{0, \ldots, K\}$ we let $D_{k} \subseteq \mathbb{R}^{\nu}$ be the set of all $y$ such that $\Pi_{m}(M)_{y}$ has exactly $k$ elements. Let $\mathcal{C}$ be a $C^{p}$-cell decomposition of $\mathbb{R}^{m}$ 
compatible with $\Pi_{m}(M)$ such that $\mathcal{D}:=\left\{\Pi_{\nu}(C): C \in \mathcal{C}\right\}$ is a stratification compatible with $D_{0}, \ldots, D_{K}$, and let $C \in \mathcal{C}$ be such that $C \subseteq \Pi_{m}(M)$.

Claim: There are open $U_{1}, \ldots, U_{l} \subseteq U$ and definable $C^{p}$-maps $f_{i}: U_{i} \longrightarrow$ $\mathbb{R}^{m-\nu}$ such that gr $f_{i} \subseteq \Pi_{m}(M)$ for each $i$ and $C \subseteq \operatorname{gr} f_{1} \cup \cdots \cup \operatorname{gr} f_{l}$.

Assuming the claim, we obtain a finite covering of $\Pi_{m}(M)$ by finitely many definable $C^{p}$-submanifolds $V$ of $\mathbb{R}^{m}$ of dimension $\nu$. For each such $V$, we let $N$ be the set of all $x \in M$ for which there exists an open neighbourhood $M_{x}$ in $M$ such that $\Pi_{m}\left(M_{x}\right) \subseteq V$. By the Rank Theorem, $N$ is an open subset of $M$ and $\Pi_{m}(N)=V$, so the lemma is proved.

To see the claim, we write $C=\operatorname{gr} g$ with $g: D \longrightarrow \mathbb{R}^{m-\nu}$ definable and $C^{p}$ and $D:=\Pi_{\nu}(C) \in \mathcal{D}$. We also put $\mathcal{D}_{D}:=\left\{D^{\prime} \in \mathcal{D}: D \subseteq\right.$ fr $\left.D^{\prime}\right\}$ and $\mathcal{C}_{D}:=$ $\left\{C^{\prime} \in \mathcal{C}: \Pi_{\nu}\left(C^{\prime}\right) \in \mathcal{D}_{D}\right.$ and $\left.C^{\prime} \subseteq \Pi_{m}(M)\right\}$, and we call a map $s: \mathcal{D}_{D} \longrightarrow \mathcal{C}_{D}$ a section if $\Pi_{\nu}\left(s\left(D^{\prime}\right)\right)=D^{\prime}$ for every $D^{\prime} \in \mathcal{D}_{D}$. To every section $s: \mathcal{D}_{D} \longrightarrow$ $\mathcal{C}_{D}$, we associate a set

$$
A_{s}:=\operatorname{gr} g \cup \bigcup_{D^{\prime} \in \mathcal{D}_{D}} s\left(D^{\prime}\right) ;
$$

then $A_{s}$ is the graph of a definable function $g_{s}: D \cup \cup \mathcal{D}_{D} \longrightarrow \mathbb{R}^{m-\nu}$. Since $\mathcal{D}$ is a stratification, the set $D \cup \cup \mathcal{D}_{D}$ is open, and we let $D_{s}$ be the set of all $y \in D$ such that $g_{s}$ is of class $C^{p}$ in a neighbourhood of $y$. The sets $D_{s}$ are definable open subsets of $D$, and by the hypotheses and the Rank Theorem they form a covering of $D$. Now use definable choice to obtain open definable neighbourhoods $U_{s}$ of $D_{s}$ such that $\left.g_{s}\right\rceil_{U_{s}}$ is $C^{p}$, and the claim is proved.

Next, we let $M \subseteq \mathbb{R}^{n}$ be a manifold of dimension $m \leq n$. We also let $d$ be a $p$-distribution on $M$, with $p \leq m$, and we fix $\eta>0$.

Definition 1.2. The distribution $d$ is called $\eta$-bounded at $x \in M$ if there is a matrix $L \in \mathrm{M}_{n-p, p}(\mathbb{R})$ such that $|L| \leq \eta$ and $d(x)=\left\{(u, L u): u \in \mathbb{R}^{p}\right\}$. The distribution $d$ is $\eta$-bounded if $d$ is $\eta$-bounded at every $x \in M$.

Remark. If $d$ is $\eta$-bounded, then every integral manifold of $d$ is $\eta$-bounded (as defined in our conventions).

Given a permutation $\sigma \in \Sigma_{n}$, the set $\sigma^{-1}(M)$ is a manifold and the pull-back $\sigma^{*} d$ is a distribution on $\sigma^{-1}(M)$; we define

$$
M_{\sigma, \eta}:=\left\{x \in M: \sigma^{*} d \text { is } \eta \text {-bounded at } \sigma^{-1}(x)\right\} .
$$

Note that $M_{\sigma, \eta}$ is open in $M$.

Lemma 1.3. (1) If $\eta>1$, then $M=\bigcup_{\sigma \in \Sigma_{n}} M_{\sigma, \eta}$.

(2) If $M$ is definable, then so is each $M_{\sigma, \eta}$. 
Proof. Part (1) follows from the following elementary observation (see Lemma 3 of [14] for details): let $E \subseteq \mathbb{R}^{n}$ be a linear subspace of dimension $p$. Then there exist $\sigma \in \Sigma_{n}$ and $L \in M_{n-p, p}(\mathbb{R})$ such that $|L| \leq 1$ and

$$
\sigma^{-1}(E)=\left\{(u, L u) \in \mathbb{R}^{n}: u \in \mathbb{R}^{p}\right\} .
$$

For part (2), note that the set $\mathcal{E}_{\eta}$, consisting of all $E \in G_{n}^{p}$ for which there exists an $L \in M_{n-p, p}(\mathbb{R})$ such that $|L|<\eta$ and $E=\left\{(u, L u): u \in \mathbb{R}^{p}\right\}$, is open and semialgebraic.

The next two lemmas are crucial tools in our use of Hausdorff limits. For $x \in \mathbb{R}^{n}$ and $p \leq n$, we set $x_{\leq p}:=\left(x_{1}, \ldots, x_{p}\right)$ and $x_{>p}:=\left(x_{p+1}, \ldots, x_{n}\right)$. Recall that we are working in the topology of the norm $|\cdot|$ below.

Lemma 1.4. Let $\eta>0$, and let $V$ be an $\eta$-bounded submanifold of $M$ of dimension $p \leq m$. Let $x \in V$, and let $\epsilon>0$ be such that

$$
\left(B\left(x_{\leq p}, \epsilon\right) \times B\left(x_{>p}, p \eta \epsilon\right)\right) \cap \operatorname{fr} V=\emptyset .
$$

Then the component of $V \cap\left(B\left(x_{\leq p}, \epsilon\right) \times B\left(x_{>p}, p \eta \epsilon\right)\right)$ that contains $x$ is the

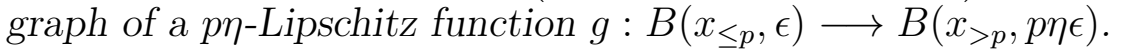

Proof. We set $W:=B\left(x_{\leq p}, \epsilon\right)$ and $W^{\prime}:=B\left(x_{>p}, p \eta \epsilon\right)$, and we denote by $C$ the component of $V \cap\left(W \times W^{\prime}\right)$ that contains $x$. Since $C$ is $\eta$-bounded, the map $\Pi_{p} \uparrow_{C}: C \longrightarrow W$ is a local homeomorphism onto its image. By general topology, it is therefore enough to show that $\Pi_{p}(C)=W$; we do this by showing that there is a function $g: W \longrightarrow W^{\prime}$ such that gr $g \subseteq C$.

Since $V$ is $\eta$-bounded, there are $\delta>0$ and a $p \eta$-Lipschitz function $g$ : $B\left(x_{\leq p}, \delta\right) \longrightarrow W^{\prime}$ such that gr $g \subseteq C$. We extend $g$ to all of $W$ as follows: for $v \in \mathrm{bd} W$, we let $v^{\prime}$ be the point in the closed line segment $\left[x_{\leq p}, v\right]$ closest to $v$ such that $g$ extends to a $p \eta$-Lipschitz function $g_{v}$ along the half-open line segment $\left[x_{\leq p}, v^{\prime}\right)$ satisfying gr $g_{v} \subseteq V \cap\left(W \times W^{\prime}\right)$. Then the proportion of the sidelengths of $W$ and $W^{\prime}$, the $\eta$-boundedness of $V$ and the fact that $\left(W \times W^{\prime}\right) \cap$ fr $V=\emptyset$ imply that $v^{\prime}=v$ for all $v \in$ bd $W$. Since the graph of the resulting function $g: W \longrightarrow W^{\prime}$ is connected and contains $x$, it follows that gr $g \subseteq C$, as required.

Lemma 1.5. Let $\left(V_{\iota}\right)$ be a sequence of submanifolds of $M$ of dimension $p \leq$ $m$. Let $\eta>0$, and assume that each $V_{\iota}$ is $\eta$-bounded. Moreover, assume that both $\lim _{\iota} V_{\iota}$ and $\lim _{\iota}$ fr $V_{\iota}$ exist and there is an $\nu \in \mathbb{N}$ such that for every $\iota$ and every open box $U \subseteq \mathbb{R}^{n}$, the set $V_{\iota} \cap U$ has at most $\nu$ components. Then for every $x \in \lim _{\iota} V_{\iota} \backslash \lim _{\iota}$ fr $V_{\iota}$, there are a box $U \subseteq \mathbb{R}^{n}$ containing $x$ and $p \eta$-Lipschitz functions $f_{1}, \ldots, f_{\nu}: \Pi_{p}(U) \longrightarrow \mathbb{R}^{n-p}$ such that

$$
\lim _{\iota} V_{\iota} \cap U=\left(\operatorname{gr} f_{1} \cap U\right) \cup \cdots \cup\left(\operatorname{gr} f_{\nu} \cap U\right) .
$$


Proof. We write "lim" in place of "lim $\lim V_{\iota} \backslash \lim$ fr $V_{\iota}$, and choose $\epsilon>0$ such that $\left(B\left(x_{\leq p}, 3 \epsilon\right) \times B\left(x_{>p}, 3 p \eta \epsilon\right)\right) \cap$ fr $V_{\iota}=\emptyset$ for all $\iota$ (after passing to a subsequence if necessary). We let $U:=$ $B\left(x_{\leq p}, \epsilon\right) \times B\left(x_{>p}, p \eta \epsilon\right), W:=B\left(x_{\leq p}, \epsilon\right)$ and $W^{\prime}:=B\left(x_{>p}, 3 p \eta \epsilon\right)$. Then for each $\iota$, the assumptions and Lemma 1.4 imply, with $2 \epsilon$ in place of $\epsilon$ and each $z \in U \cap V_{\iota}$ in place of $x$, that there are definable $p \eta$-Lipschitz functions $f_{1, \iota}, \ldots, f_{\nu, \iota}: W \longrightarrow \mathbb{R}^{n-p}$ such that every component of $V_{\iota} \cap\left(W \times W^{\prime}\right)$ intersecting $U$ is the graph of some $f_{\lambda, \iota}$. Moreover, we have either $f_{\lambda, \iota}=f_{\lambda^{\prime}, \iota}$ or gr $f_{\lambda, \iota} \cap \operatorname{gr} f_{\lambda^{\prime}, \iota}=\emptyset$, for all $\lambda, \lambda^{\prime} \in\{1, \ldots, \nu\}$, and

$$
V_{\iota} \cap U=\left(\operatorname{gr} f_{1, \iota} \cap U\right) \cup \cdots \cup\left(\operatorname{gr} f_{\nu, \iota} \cap U\right) .
$$

Passing to a subsequence if necessary, we may assume that each sequence $\left(f_{\lambda, \iota}\right)_{\iota}$ converges to a $p \eta$-Lipschitz function $f_{\lambda}: W \longrightarrow \mathbb{R}^{n-p}$; then gr $f_{\lambda} \subseteq \lim V_{\iota}$. On the other hand, if $x^{\prime} \in \lim V_{\iota} \cap U$, then $x^{\prime} \in \lim \left(V_{\iota} \cap U\right)$, so by the above $x^{\prime} \in \lim \left(\operatorname{gr} f_{\lambda, \iota} \cap U\right)$ for some $\lambda$, that is, $x^{\prime} \in \operatorname{gr} f_{\lambda}$.

The following lemma is also central to our use of Hausdorff limits. We recall that a function $\phi: M \longrightarrow(0, \infty)$ is a carpeting function on $M$ if $\phi$ is proper and satisfies $\lim _{x \rightarrow y} \phi(x)=0$ whenever $y \in$ fr $M$, where the frontier is taken inside the one-point compactification $\mathbb{R}^{n} \cup\{\infty\}$ of $\mathbb{R}^{n}$. For instance, given positive real numbers $u_{1}, \ldots, u_{n}$, the function

$$
x \mapsto \phi_{u}(x):=\frac{1}{1+u_{1} x_{1}^{2}+\cdots+u_{n} x_{n}^{2}}
$$

is a real analytic carpeting function on $\mathbb{R}^{n}$.

Lemma 1.6. Assume that $M$ is bounded and has a carpeting function $\phi$. Let $V$ be a closed subset of $M$, and assume that $V \cap U$ has finitely many components for every open box $U \subseteq \mathbb{R}^{n}$. Then for every sequence $\left(r_{\kappa}\right)_{\kappa \in \mathbb{N}}$ of positive real numbers satisfying $r_{\kappa} \rightarrow 0$ as $\kappa \rightarrow \infty$, we have

$$
\text { fr } V=\lim _{\kappa}\left(V \cap \phi^{-1}\left(r_{\kappa}\right)\right) \text {. }
$$

Proof. Let $r_{\kappa} \rightarrow 0$ as $\kappa \rightarrow \infty$. It suffices to show that

$$
\text { fr } V=\lim _{j}\left(\phi^{-1}\left(r_{\kappa(j)}\right) \cap V\right)
$$

for every subsequence $(\kappa(j))_{j \in \mathbb{N}}$ of $(\kappa)$ such that the limit on the right-hand side exists in $\mathcal{K}_{n}$, that is, we may assume that the sequence $\left(\phi^{-1}\left(r_{\kappa}\right) \cap V\right)$ converges in $\mathcal{K}_{n}$. The properties of $\phi$ then imply that fr $V \supseteq \lim _{\kappa}\left(\phi^{-1}\left(r_{\kappa}\right) \cap V\right)$. Conversely, let $x \in \operatorname{fr} V$. Since $V \cap B(x, 1)$ has finitely many components, there is a component $C$ of $V \cap B(x, 1)$ such that $x \in \operatorname{fr} C$. Then $C \cup\{x\}$ is connected, so there is a continuous curve $\gamma:[0,1] \longrightarrow C \cup\{x\}$ such that $\gamma([0,1)) \subseteq C$ and $\gamma(1)=x$. Hence $\phi \circ \gamma:[0,1) \longrightarrow(0, \infty)$ is continuous and satisfies $\lim _{t \rightarrow 1} \phi(\gamma(t))=0$, so the intermediate value theorem implies 
that the image $\gamma([0,1))$ intersects $\phi^{-1}\left(r_{\kappa}\right)$ for all sufficiently large $\kappa$, so that $x \in \lim _{\kappa}\left(\phi^{-1}\left(r_{\kappa}\right) \cap V\right)$. Hence fr $V=\lim _{\kappa}\left(\phi^{-1}\left(r_{\kappa}\right) \cap V\right)$.

Definition 1.7. We abbreviate the conclusion of the lemma by the statement

$$
\operatorname{fr} V=\lim _{r \rightarrow 0}\left(V \cap \phi^{-1}(r)\right) .
$$

\section{Nested Distributions}

We fix an o-minimal expansion $\mathcal{R}$ of the real field. The goal of this section and the next is to develop Khovanskii theory for nested pfaffian sets over $\mathcal{R}$. We closely follow the exposition of Sections 1 and 2 in [20].

Let $M \subseteq \mathbb{R}^{n}$ be a $C^{2}$-manifold of dimension $m$.

Definition $2.1([18])$. Let $\mathcal{D}$ be a set of distributions on $M$. A $C^{2}$-submanifold $N$ of $M$ is compatible with $\mathcal{D}$ if the pull-back $\left(\bigcap_{e \in \mathcal{E}} e\right)^{N}$ has dimension for every $\mathcal{E} \subseteq \mathcal{D}$. A collection $\mathcal{C}$ of $C^{2}$-submanifolds of $M$ is compatible with $\mathcal{D}$ if every $C \in \mathcal{C}$ is compatible with $\mathcal{D}$.

Proposition 2.2. Let $A_{1}, \ldots, A_{k} \subseteq \mathbb{R}^{n}$ be definable and $p \geq 2$, and assume that $M$ is definable and $\mathcal{D}$ is a finite set of definable distributions on $M$. Then there is a finite partition (stratification, Whitney stratification) $\mathcal{P}$ of $M$ into definable $C^{p}$-cells such that $\mathcal{P}$ is compatible with each $A_{j}$ as well as $\mathcal{D}$.

Proof. We proceed by induction on $m=\operatorname{dim} M$; the case $m=0$ is trivial. So we assume $m>0$ and the lemma holds for lower values of $m$. By $C^{2}$ cell decomposition, we may assume that $A_{1}, \ldots, A_{k}$ is a partition of $M$ into definable $C^{2}$-cells. Thus, for $x \in M$, there is a unique $l(x) \in\{1, \ldots, k\}$ such that $x \in A_{l(x)}$.

For $x \in M$ and $\mathcal{E} \subseteq \mathcal{D}$ we set $T_{x} \mathcal{E}:=T_{x} A_{l(x)} \cap \bigcap_{d \in \mathcal{E}} d(x)$. For $\mathcal{E} \subseteq \mathcal{D}$, $j \in\{1, \ldots, k\}$ and $i \in\{0, \ldots, m\}$, we define the set

$$
M_{\mathcal{E}, j, i}:=\left\{x \in A_{j}: \operatorname{dim} T_{x} \mathcal{E}=i\right\} .
$$

For each $\mathcal{E}$, the sets $M_{\mathcal{E}, j, i}$ form a covering of $M$, and since each $d \in \mathcal{D}$ is definable, each set $M_{\mathcal{E}, j, i}$ is definable.

Let $\mathcal{C}$ be a partition (stratification, Whitney stratification) of $M$ into definable $C^{p}$-cells compatible with each $M_{\mathcal{E}, j, i}$. Then for $C \in \mathcal{C}$, there is a unique $j(C) \in\{1, \ldots, k\}$ such that $C \subseteq A_{j(C)}$.

Fix a $C \in \mathcal{C}$. If $\operatorname{dim} C=m$, then for $\mathcal{E} \subseteq \mathcal{D}$ there is a unique $i(C, \mathcal{E}) \in$ $\{0, \ldots, m\}$ such that $C \subseteq M_{\mathcal{E}, j(C), i(C, \mathcal{E})}$. Since $C$ is open in $M$, it follows that $\left(\bigcap_{d \in \mathcal{E}} d\right)^{C}$ has dimension $i(C, \mathcal{E})$ for every $\mathcal{E} \subseteq \mathcal{D}$. On the other hand, if $\operatorname{dim} C<m$, then the inductive hypothesis applied to $C$ and $\mathcal{D}^{C}:=\left\{d^{C}\right.$ : $d \in \mathcal{D}\}$ in place of $M$ and $\mathcal{D}$ produces a partition (stratification, Whitney 
stratification) $\mathcal{P}_{\mathcal{C}}$ of $C$ compatible with each $A_{j}$ as well as $\mathcal{D}^{C}$. Now it is straightforward to see that the collection

$$
\mathcal{P}:=\{C \in \mathcal{C}: \operatorname{dim} C=m\} \cup \bigcup_{C \in \mathcal{C}, \operatorname{dim} C<m} \mathcal{P}_{\mathcal{C}}
$$

is a partition (stratification, Whitney stratification) of $M$ compatible with each $A_{j}$ as well as $\mathcal{D}$ (we leave the details to the reader).

Definition 2.3. Let $d$ be a distribution on $M$ that has dimension. Then $d$ gives rise to a vector subbundle $T_{M} d$ of $T M$. A section $v: M \longrightarrow T M$ is a vector field on $M ; v$ is tangent to $d$ if $v(M) \subseteq T_{M} d$ (or equivalently, if $v(x) \in d(x)$ for all $x \in M)$. Let $\mathcal{V}^{1}(M, d)$ be the collection of all vector fields on $M$ tangent to $d$, and put

$$
I(d):=\left\{x \in M:[v, w](x) \in d(x) \text { for all } v, w \in \mathcal{V}^{1}(M, d)\right\},
$$

where $[v, w]$ denotes the Lie bracket of the vector fields $v$ and $w$. We say that $d$ is integrable if $I(d)=M$ and that $d$ is nowhere integrable if $I(d)=\emptyset$.

Remarks 2.4. Let $d$ be a distribution on $M$ that has dimension.

(1) The Gauss map $g_{M}$ is integrable.

(2) Every 1-distribution on $M$ is integrable.

(3) Let $e$ be a distribution on $M$ such that $e$ and $d \cap e$ have dimension. Then $I(d) \cap I(e) \subseteq I(d \cap e)$. In particular, if $d$ and $e$ are integrable, then $d \cap e$ is integrable.

(4) Let $N$ be a $C^{2}$-submanifold of $M$ compatible with $d$. Then $I(d) \cap N \subseteq$ $I\left(d^{N}\right)$; in particular, if $d$ is integrable, so is $d^{N}$.

(5) If $M$ and $d$ are definable, then the set $I(d)$ is definable.

(6) Let $V$ be an integral manifold of $d$. Then $V \subseteq I(d)$.

Let $g$ be an integrable $p$-distribution on $M$ with $0 \leq p \leq m$. Then by the theorem of Frobenius (see for instance Camacho and Lins Neto [2, p. 36]), every $x \in M$ belongs to a unique leaf $L_{x}^{g}$ of $g$, and there is an equivalence relation $\sim_{g}$ on $M$ associated to $g$ given by

$$
x \sim_{g} y \quad \text { iff } \quad L_{x}^{g}=L_{y}^{g} .
$$

Clearly, if $\sim_{g}$ is definable, then so is $g$; however, the converse is not true in general.

For the rest of this section, we assume that $M$ is definable, and we fix a definable nested distribution $d=\left(d_{0}, \ldots, d_{k}\right)$ on $M$.

Definitions 2.5. (1) If $N$ is a $C^{2}$-submanifold of $M$, we say that $N$ is compatible with $d$ if $N$ is compatible with the set $\left\{d_{0}, \ldots, d_{k}\right\}$.

(2) Let $N$ be a $C^{2}$-submanifold of $M$ compatible with $d$. Then $\operatorname{dim} d_{i}^{N} \leq$ $\operatorname{dim} d_{i-1}^{N} \leq 1+\operatorname{dim} d_{i}^{N}$ for $i=1, \ldots, k$. Thus, we define the pull-back 
$d^{N}$ of $d$ on $N$ as the nested distribution on $N$ obtained by listing the set $\left\{d_{0}^{N}, \ldots, d_{k}^{N}\right\}$ in decreasing order of dimension.

(3) We call $d$ integrable if each $d_{i}$ is integrable, and we call $d$ nowhere integrable if each $d_{i}$ is nowhere integrable.

(4) We put $\operatorname{dim} d:=m-k$, and if $d$ is integrable, we define

$$
\operatorname{deg} d:=\mid\left\{i \in\{0, \ldots, k\}: \sim_{d_{i}} \text { is not definable }\right\} \mid \leq k,
$$

called the degree of $d$, where $|\cdot|$ denotes cardinality.

Remarks. (1) If $N$ is a $C^{2}$-submanifold of $M$ compatible with $d$, then $\operatorname{deg} d^{N} \leq \operatorname{deg} d$.

(2) Put $d^{\prime}:=\left(d_{0}, \ldots, d_{k-1}\right)$. If $\sim_{d_{k}}$ is not definable, then $\operatorname{deg} d^{\prime}<\operatorname{deg} d$.

We assume for the rest of this section that $d$ is integrable.

Definition 2.6. Let $e=\left(e_{0}, \ldots, e_{l}\right)$ be an integrable, definable nested distribution on $M$ with $l \leq k$. We call $e$ a core distribution of $d$ if

(i) $\sim_{d_{i}}$ is definable for $i=1, \ldots, k-l$, and

(ii) $d_{i}=d_{k-l} \cap e_{i-k+l}$ for $i=k-l+1, \ldots, k$.

Remarks. (1) Let $e$ be a core distribution of $d$. Then $\operatorname{deg} d \leq \operatorname{deg} e$. Moreover, if $f$ is a core distribution of $e$, then $f$ is also a core distribution of $d$.

(2) Let $N$ be a definable $C^{2}$-submanifold of $M$ compatible with $d$, and let $e$ be a core distribution of $d$. If $N$ is compatible with $e$, then $d^{N}$ has core distribution $e^{N}$.

In our setting, core distributions typically arise in the following way:

Example 2.7. Let $\phi: M \longrightarrow \mathbb{R}$ be definable and $C^{2}$, and define $g_{\phi}: M \longrightarrow$ $G_{n}$ by $g_{\phi}(x):=\operatorname{ker} d \phi(x) \subseteq T_{x} M$; note that $g_{\phi}(x)$ has codimension at most 1 in $T_{x} M$. We set $\mathcal{D}_{\phi}:=\left\{g_{M}, g_{\phi}, d_{1} \cap g_{\phi}, \ldots, d_{k} \cap g_{\phi}\right\}$, and for a $C^{2}$-submanifold $N$ of $M$ compatible with $\mathcal{D}_{\phi}$, we let $d_{\phi}^{N}$ be the definable nested distribution on $N$ obtained by listing the pull-backs of all elements of $\mathcal{D}_{\phi}$ to $N$ in order of decreasing dimension. If, in addition, $N$ is compatible with $d$, then $d_{\phi}^{N}$ has core distribution $d^{N}$ and $\operatorname{dim} d^{N} \geq \operatorname{dim} d_{\phi}^{N} \geq \operatorname{dim} d^{N}-1$; in particular, $\operatorname{deg} d_{\phi}^{N} \leq \operatorname{deg} d$.

We now let $\mathcal{C}$ be a Whitney stratification of $M$ by definable $C^{2}$-cells compatible with both $d$ and $\mathcal{D}_{\phi}$, as obtained from Proposition 2.2. Let $\mathcal{C}^{\prime}$ be the set of all $C \in \mathcal{C}$ such that $g_{C} \cap d_{k} \nsubseteq g_{\phi}$. Then $\operatorname{dim} d_{\phi}^{C}<\operatorname{dim} d$ for $C \in \mathcal{C}^{\prime}$, and we claim that the union of all cells in $\mathcal{C}^{\prime}$ is an open subset $M^{\prime}$ of $M$. To see this, note first that if $C, D \in \mathcal{C}$ are such that $D \subseteq \operatorname{fr} C$, then the Whitney property of the pair $(C, D)$, as defined on p. 502 of [5], implies that for every sequence $\left(x_{i}\right)_{i \in \mathbb{N}}$ of points in $C$ that converges to a point $y \in D$ and for which $T:=\lim _{i} T_{x_{i}} C$ exists in $G_{n}^{\operatorname{dim} C}$, we have $T_{y} D \subseteq T$. Since $d$ and $g_{\phi}$ are 
continuous, it follows that the union of all cells in $\mathcal{C} \backslash \mathcal{C}^{\prime}$ is a closed subset of $M$; hence $M^{\prime}$ is an open subset of $M$. Finally, note that $d_{k}(x) \nsubseteq g_{\phi}(x)$ for all $x \in M^{\prime}$, so that $M^{\prime}$ is compatible with $\mathcal{D}_{\phi}$ and $\operatorname{dim} d_{\phi}^{M^{\prime}}<\operatorname{dim} d^{M^{\prime}}$.

\section{KHOVANSKII THEORY}

Let $M \subseteq \mathbb{R}^{n}$ be a definable $C^{2}$-manifold of dimension $m$. We fix a finite family $\Delta=\left\{d^{1}, \ldots, d^{q}\right\}$ of definable nested distributions on $M$; we write $d^{p}=\left(d_{0}^{p}, \ldots, d_{k(p)}^{p}\right)$ for $p=1, \ldots, q$. We associate to $\Delta$ the following set of distributions on $M$ :

$$
\mathcal{D}_{\Delta}:=\left\{d_{0}^{0} \cap d_{k(1)}^{1} \cap \cdots \cap d_{k(p-1)}^{p-1} \cap d_{j}^{p}: p=1, \ldots, q \text { and } j=0, \ldots, k(p)\right\},
$$

where $d_{0}^{0}=g_{M}$. If $N$ is a $C^{2}$-submanifold of $M$ compatible with $\mathcal{D}_{\Delta}$, we let $d^{\Delta, N}=\left(d_{0}^{\Delta, N}, \ldots, d_{k, \Delta, N)}^{\Delta, N}\right)$ be the nested distribution on $N$ obtained by listing the set $\left\{g^{N}: g \in \mathcal{D}_{\Delta}\right\}$ in order of decreasing dimension. In this situation, if $V_{p}$ is an integral manifold of $d_{k(p)}^{p}$, for $p=1, \ldots, q$, then the set $N \cap V_{1} \cap \cdots \cap V_{q}$ is an integral manifold of $d_{k(\Delta, N)}^{\Delta, N}$.

Lemma 3.1. Let $N \subseteq M$ be a definable $C^{2}$-cell compatible with $\mathcal{D}_{\Delta}$, and suppose that $\operatorname{dim} d_{k(\Delta, N)}^{\Delta, N}>0$. Then there is a definable carpeting function $\phi$ on $N$ of class $C^{2}$ such that the definable set

$$
B:=\left\{x \in N: d_{k(\Delta, N)}^{\Delta, N}(x) \subseteq \operatorname{ker} d \phi(x)\right\}
$$

has dimension less than $\operatorname{dim} N$.

Proof. By [5] there is a definable diffeomorphism $\sigma: \mathbb{R}^{\operatorname{dim} N} \longrightarrow N$ of class $C^{2}$. Replacing $n$ by $\operatorname{dim} N, N$ by $\mathbb{R}^{\operatorname{dim} N}$ and each $d_{i}^{\Delta, N}$ by its pull-back $\sigma^{*} d_{i}^{\Delta, N}$, we reduce to the case where $N=M=\mathbb{R}^{n}$ and write $k$ and $d$ in place of $k(\Delta, N)$ and $d^{\Delta, N}$. Then for $u=\left(u_{1}, \ldots, u_{n}\right) \in(0, \infty)^{n}$ we put

$$
B_{u}:=\left\{x \in \mathbb{R}^{n}: d_{k}(x) \subseteq \operatorname{ker} d \phi_{u}(x)\right\},
$$

where $\phi_{u}$ is the carpeting function defined on $\mathbb{R}^{n}$ before Lemma 1.6. If $\operatorname{dim} B_{u}<n$ for some $u$ as above, the proof is finished. So assume for a contradiction that $\operatorname{dim} B_{u}=n$ for all $u$ as above. Then $\operatorname{dim} B=2 n$, where

$$
B:=\left\{(u, x) \in(0, \infty)^{n} \times \mathbb{R}^{n}: x \in B_{u}\right\},
$$

so there are nonempty open $V \subseteq(0, \infty)^{n}$ and $W \subseteq \mathbb{R}^{n}$ such that $V \times W \subseteq B$. Fix some $x \in W$ with all $x_{i} \neq 0$ and let $u$ range over $V$. Note that

$$
d \phi_{u}(x)=-\phi_{u}(x)^{2}\left(2 u_{1} x_{1} d x_{1}+\cdots+2 u_{n} x_{n} d x_{n}\right) .
$$

Therefore the vector space generated by all $d \phi_{u}(x)$ as $u$ ranges over $V$ has dimension $n$, that is, the intersection of all ker $d \phi_{u}(x)$ as $u$ ranges over $V$ is trivial, which contradicts $\operatorname{dim} d>0$. 
For convenience, if $e=\left(e_{0}, \ldots, e_{l}\right)$ is a nested distribution on $M$ and $W \subseteq$ $M$, we call $W$ a Rolle leaf of $e$ if there is a nested Rolle leaf $V=\left(V_{0}, \ldots, V_{l}\right)$ of $e$ such that $W=V_{l}$.

Proposition 3.2. Let $A \subseteq \mathbb{R}^{n}$ be a definable set. Then there exists a $K \in \mathbb{N}$ such that, whenever $L_{p}$ is a Rolle leaf of $d^{p}$ for $p=1, \ldots, q$, then $A \cap L_{1} \cap \cdots \cap L_{q}$ is a union of at most $K$ connected manifolds.

Proof. We proceed by induction on $\operatorname{dim} A$ and $k:=k(1)+\cdots+k(q)$. The cases $\operatorname{dim} A=0$ or $k=0$ being trivial, we assume that $\operatorname{dim} A>0$ and $k>0$ and that the result holds for lower values of $\operatorname{dim} A$ or $k$. After shrinking $q$, we may also assume that $k(p)>0$ for each $p$. By Proposition 2.2, it suffices to consider the case where $A=N$ is a $C^{2}$-cell contained in $M$ and compatible with $\mathcal{D}_{\Delta}$. For $p=1, \ldots, q$, we let $L_{p}$ be a Rolle leaf of $d^{p}$, and we put $L:=L_{1} \cap \cdots \cap L_{q}$; then $N \cap L$ is an integral manifold of $d:=d_{k(\Delta, N)}^{\Delta, N}$.

Case $\operatorname{dim} d=0$. Let $\Delta^{\prime}:=\left\{d^{1}, \ldots, d^{q-1},\left(d_{0}^{q}, \ldots, d_{k(q)-1}^{q}\right)\right\}$, let $L_{q}^{\prime}$ be the Rolle leaf of $d_{k(q)-1}^{q}$ containing $L_{q}$ and put $L^{\prime}:=L_{1} \cap \cdots \cap L_{q-1} \cap L_{q}^{\prime}$. Then $N$ is compatible with $\mathcal{D}_{\Delta^{\prime}}$ and $N \cap L^{\prime}$ is an integral manifold of $d^{\prime}:=d_{k\left(\Delta^{\prime}, N\right)}^{\Delta^{\prime}, N}$ of dimension at most 1. By the inductive hypothesis, there is a $K \in \mathbb{N}$ (depending only on $N$ and $\Delta^{\prime}$, but not on the particular Rolle leaves) such that the manifold $N \cap L^{\prime}$ has at most $K$ components. Thus, if $\operatorname{dim}\left(N \cap L^{\prime}\right)=0$, we are done by the inductive hypothesis, so we assume that $\operatorname{dim}\left(N \cap L^{\prime}\right)=1$. Since $N$ is compatible with $\mathcal{D}_{\Delta^{\prime}}$, it follows that $\operatorname{dim} d^{\prime}=1$ as well.

Let $C$ be a component of $N \cap L^{\prime}$. If $C \cap L_{q}$ contains more than one point, then by the Rolle property of $L_{q}$ in $L_{q}^{\prime}$ and the fact that $C$ is a connected $C^{1}$-submanifold of $L_{q}^{\prime}$ of dimension $1, C$ is tangent at some point to $\left(d_{k(q)}^{q}\right)^{L_{q}^{\prime}}$, which contradicts the assumption that $\operatorname{dim} d=0$. So $C \cap L_{q}$ contains at most one point for each component $C$ of $N \cap L^{\prime}$. Hence $N \cap L$ consists of at most $K$ points.

Case $\operatorname{dim} d>0$. Let $\phi$ and $B$ be obtained from Lemma 3.1. Then $\operatorname{dim} B<$ $\operatorname{dim} A$; so by the inductive hypothesis, there is a $K \in \mathbb{N}$, independent of the particular Rolle leaves chosen, such that $B \cap L$ has at most $K$ components. Since $N \cap L$ is a closed, embedded submanifold of $N, \phi$ attains a maximum on every component of $N \cap L$, and any point in $N \cap L$ where $\phi$ attains a local maximum belongs to $B$. Hence $N \cap L$ has at most $K$ components.

Corollary 3.3. (1) Let $\mathcal{C}$ be a partition of $M$ into definable $C^{2}$-cells compatible with $\mathcal{D}_{\Delta}$. Then there is a $K \in \mathbb{N}$ such that, for every $C \in \mathcal{C}$ and every Rolle leaf $L_{p}$ of $d^{p}$ with $p=1, \ldots, q$, the set $C \cap L_{1} \cap \cdots \cap L_{q}$ is a union of at most $K$ Rolle leaves of $d^{\Delta, C}$. 
(2) Let $\mathcal{A}$ be a definable family of sets. Then there is a $K \in \mathbb{N}$ such that whenever $A \in \mathcal{A}$ and $L_{p}$ is a Rolle leaf of $d^{p}$ for each $p$, the set $A \cap L_{1} \cap \cdots \cap L_{q}$ is a union of at most $K$ connected manifolds.

Proof. Part (1) follows from the proof of Proposition 3.2. For (2), we let $A \subseteq \mathbb{R}^{m+n}$ be definable such that $\mathcal{A}=\left\{A_{z}: z \in \mathbb{R}^{m}\right\}$ where, for $z \in \mathbb{R}^{m}$, $A_{z}:=\left\{x \in \mathbb{R}^{n}:(z, x) \in A\right\}$. We replace $M$ by $M^{\prime}:=\mathbb{R}^{m} \times M$ and each $d_{i}^{p}$ by the distribution $e_{i}^{p}$ on $M^{\prime}$ defined by $e_{i}^{p}(z, x):=\mathbb{R}^{m} \times d_{i}^{p}(x)$. Moreover, we let $e$ be the nested distribution on $\mathbb{R}^{m+n}$ obtained as in Example 1 from the family $\Omega=\left(d z_{1}, \ldots, d z_{m}\right)$, and we let $e^{q+1}$ be the restriction of $e$ to $M^{\prime}$ and put $\Delta^{\prime}:=\left\{e^{1}, \ldots, e^{q+1}\right\}$. By Proposition 3.2 , there is a $K^{\prime}$ such that whenever $L_{p}^{\prime}$ is a Rolle leaf of $e^{p}$, for $p=1, \ldots, q+1$, then $A \cap L_{1}^{\prime} \cap \cdots \cap L_{q+1}^{\prime}$ is the union of at most $K^{\prime}$ connected manifolds. But for every Rolle leaf $L_{p}$ of $d^{p}$, with $p \in\{1, \ldots, q\}$, the set $\mathbb{R}^{m} \times L_{p}$ is a Rolle leaf of $e^{p}$; and for every $z \in \mathbb{R}^{m}$ and each component $C$ of $M$, the set $\{z\} \times C$ is a Rolle leaf of $e^{q+1}$. Thus, we can take $K=K^{\prime} \cdot l$, where $l$ is the number of components of $M$.

Corollary 3.4. Let $X \subseteq \mathbb{R}^{n}, X_{1} \subseteq \mathbb{R}^{n_{1}}$ and $X_{2} \subseteq \mathbb{R}^{n_{2}}$ be nested pfaffian over $\mathcal{R}$.

(1) Each component of $X$ is nested pfaffian over $\mathcal{R}$.

(2) If $n_{1}=n_{2}$, then $X_{1} \cap X_{2}$ is nested pfaffian over $\mathcal{R}$.

(3) The product $X_{1} \times X_{2}$ is nested pfaffian over $\mathcal{R}$.

Proof. Part (1) follows from Corollary [3.3(1). For (2), we may assume that $X_{1}$ and $X_{2}$ are basic nested pfaffian over $\mathcal{R}$. Let $M_{1}, M_{2} \subseteq \mathbb{R}^{n}$ be definable $C^{2}$-manifolds with $n=n_{1}=n_{2}$, and for $p=1,2$, let $d^{p}=\left(d_{0}^{p}, \ldots, d_{k(p)}^{p}\right)$ be definable nested distributions on $M_{p}, L_{p}$ be a Rolle leaf of $d^{p}$ and $A_{p} \subseteq \mathbb{R}^{n}$ be definable such that $X_{p}=A_{p} \cap L_{p}$. Let $\mathcal{C}$ be a $C^{2}$-cell decomposition of $\mathbb{R}^{n}$ compatible with $M_{1}, M_{2}, M_{1} \cap M_{2}, A_{1}$ and $A_{2}$. Refining $\mathcal{C}$ if necessary, we may also assume that if $C \in \mathcal{C}$ is such that $C \subseteq M_{1} \cap M_{2}$, then $C$ is compatible with both $d^{1}$ and $d^{2}$. Then it follows from Corollary 3.3(1) that we may assume that $M_{1}=M_{2}=C$ for each such $C \in \mathcal{C}$. In this case, we put $\Delta:=\left\{d^{1}, d^{2}\right\}$ and obtain again from Corollary 3.3 (1) that $X_{1} \cap X_{2}$ is a finite union of basic nested pfaffian sets over $\mathcal{R}$.

For (3), we argue as in the proof of Corollary $3.3(2)$, but without adding the extra nested distribution $e^{q+1}$ there, to see that $\mathbb{R}^{n_{1}} \times X_{2}$ and $X_{1} \times \mathbb{R}^{n_{2}}$ are nested pfaffian over $\mathcal{R}$. Hence $X_{1} \times X_{2}=\left(X_{1} \times \mathbb{R}^{n_{2}}\right) \cap\left(\mathbb{R}^{n_{1}} \times X_{2}\right)$ is nested pfaffian over $\mathcal{R}$ by part (2).

Corollary 3.5. Let $A \subseteq M$ be a definable set and $d=\left(d_{0}, \ldots, d_{k}\right)$ be a definable nested distribution on $M$. Let $p \in \mathbb{N} \cup\{\infty, \omega\}$ with $p \geq 2$, and assume that $\mathcal{R}$ admits $C^{p}$-cell decomposition. Then there are $N, s \in \mathbb{N}$ and 
18

a collection $\left\{\left(C_{j}, \psi_{j}, e^{j}\right): j=1, \ldots, s\right\}$ such that $\left\{C_{1}, \ldots, C_{s}\right\}$ is a $C^{p}$-cell decomposition of $A$ and for $j=1, \ldots, s$,

(i) $\psi_{j}: \mathbb{R}^{n_{j}} \longrightarrow C_{j}$ is a definable $C^{p}$-diffeomorphism, where $n_{j}:=\operatorname{dim} C_{j}$;

(ii) $e^{j}=\left(e_{0}^{j}, \ldots, e_{k(j)}^{j}\right)$ is a definable nested distribution on $\mathbb{R}^{n_{j}}$ of class $C^{p-1}$

(iii) whenever $V$ is a Rolle leaf of $d_{k}$, there are (not necessarily pairwise distinct) Rolle leaves $V_{r}^{j}$ of $e^{j}$, for $j=1, \ldots, s$ and $r=1, \ldots, N$, such that $A \cap V=\bigcup_{j, r} \psi_{j}\left(V_{r}^{j}\right)$.

Proof. Let $\mathcal{C}$ be a definable $C^{p}$-cell decomposition of $\mathbb{R}^{n}$ compatible with $M$, $A$ and $d$. Note that every $C \in \mathcal{C}$ is definably $C^{p}$-diffeomorphic to $\mathbb{R}^{\operatorname{dim} C}$. The corollary now follows from Corollary $3.3(1)$.

Finally, we address the issue of definability in the pfaffian closure $\mathcal{P}(\mathcal{R})$ of $\mathcal{R}$; we adopt here the terminology of Section 4 in [20].

Proposition 3.6. Let $W$ be a Rolle leaf over $\mathcal{P}(\mathcal{R})$. Then $W$ is definable in $\mathcal{P}(\mathcal{R})$.

Proof. Let $N \subseteq \mathbb{R}^{n}$ be a $C^{2}$-manifold definable in $\mathcal{P}(\mathcal{R})$, and let $d=\left(d_{0}, \ldots, d_{k}\right)$ be a nested distribution on $N$ definable in $\mathcal{P}(\mathcal{R})$ such that $W$ is a Rolle leaf of $d$. We proceed by induction on $k$; the case $k=0$ is trivial, so we assume $k>0$ and the proposition holds for lower values of $k$. By definition of $\mathcal{P}(\mathcal{R})$ and $\mathcal{R}_{i}$, there is an $i \in \mathbb{N}$ such that $N$ and $d$ are definable in $\mathcal{R}_{i}$. By Corollary 3.5, with $\mathcal{R}_{i}$ in place of $\mathcal{R}$, we may assume that $N=\mathbb{R}^{n}$. Let $\Omega$ be associated to $d$ as in Example 1 (with $\mathbb{R}^{n}$ in place of $M$ ), and let $V=\left(V_{0}, \ldots, V_{k}\right.$ ) be a nested Rolle leaf of $d$ such that $W=V_{k}$. Then $V_{1} \in \mathcal{L}\left(\mathcal{R}_{i}\right)$, so $V_{1}$ is definable in $\mathcal{R}_{i+1}$. Now let $\mathcal{C}$ be a decomposition of $V_{1}$ into $C^{2}$-cells definable in $\mathcal{R}_{i+1}$ and compatible with $d$. Then $d^{C}=\left(d_{0}^{C}, \ldots, d_{k(C)}^{C}\right)$ is definable in $\mathcal{P}(\mathcal{R})$ with $k(C)<k$, and Corollary $3.3(1)$, with $\mathcal{R}_{i+1}$ in place of $\mathcal{R}$, implies that $C \cap W$ is a finite union of Rolle leaves of $d^{C}$. The proposition therefore follows from the inductive hypothesis.

\section{Pfaffian Limits}

Let $M \subseteq \mathbb{R}^{n}$ be a bounded, definable $C^{2}$-manifold of dimension $m$ and $d=\left(d_{0}, \ldots, d_{k}\right)$ be a definable and integrable nested distribution on $M$.

Definition 4.1. A nonempty integral manifold $V$ of $d_{k}$ is an admissible integral manifold of $d$ if $d$ has a core distribution $e=\left(e_{0}, \ldots, e_{l}\right)$ and there are a definable, closed integral manifold $B=B(V)$ of $d_{k-l}$ and a Rolle leaf $W=W(V)$ of $e$ such that $V=W \cap B$. In this situation, $W$ (but not necessarily $B$ ) is uniquely determined by $V$ and $e$, and we call $W$ the core of $V$ corresponding to $e$ and $B$ a definable part of $V$ corresponding to $W$. 
Whenever they are clear from context, we shall omit the phrases "corresponding to $e$ " or "corresponding to $W$ ".

Remarks. (1) Since $d$ is integrable, an integral manifold $L$ of $d_{k}$ definable in $\mathcal{P}(\mathcal{R})$ is a leaf of $d_{k}$ if and only if $L$ is connected and closed in $M$. Hence by Corollary $3.3(1)$, every admissible integral manifold of $d$ is a closed submanifold of $M$ and a finite union of leaves of $d_{k}$.

(2) Let $V$ be an admissible integral manifold of $d$. Then there are admissible integral manifolds $V_{j}$ of $\left(d_{0}, \ldots, d_{j}\right)$, for $j=0, \ldots, k$, such that $V_{0} \supseteq \cdots \supseteq V_{k}$ and $V=V_{k}$. To see this, let $e=\left(e_{0}, \ldots, e_{l}\right), W$ and $B$ be such that $W$ is the core of $V$ corresponding to $e$ and $B$ is a definable part of $V$ corresponding to $W$. Let $\left(W_{0}, \ldots, W_{l}\right)$ be the nested Rolle leaf of $e$ such that $W=W_{l}$. Then for $j=k-l+1, \ldots, k$, we put $V_{j}:=W_{j-k+l} \cap B$, an admissible integral manifold of $d_{j}$ with core $W_{j-k+l}$ corresponding to $\left(e_{0}, \ldots, e_{j-k+l}\right)$ and definable part $B$. For $j=0, \ldots, k-l$, we let $V_{j}$ be the smallest union of leaves of $d_{j}$ containing $B$, a definable, closed integral manifold of $d_{j}$.

Remark 4.2. Let $e=\left(e_{0}, \ldots, e_{l}\right)$ be a core distribution of $d$, and let $W$ be a Rolle leaf of $e$. Let $N$ be a definable $C^{2}$-submanifold of $M$ compatible with $d$ and $e$, so that $e^{N}$ is a core distribution of $d^{N}$. By Corollary $3.3(1), W \cap N$ is a finite union of Rolle leaves $W_{1}^{N}, \ldots, W_{q}^{N}$ of $e^{N}$.

Let now $V$ be an admissible integral manifold of $d$ with core $W$ corresponding to $e$ and definable part $B$ corresponding to $W$. Then $V \cap N=V_{1}^{N} \cup \cdots \cup V_{q}^{N}$ where, for $p=1, \ldots, q$, the set $V_{p}^{N}:=W_{p}^{N} \cap B$ is an admissible integral manifold of $d^{N}$ with core $W_{p}^{N}$ corresponding to $e^{N}$ and definable part $B \cap N$.

Definition 4.3. A sequence $\left(V_{\iota}\right)_{\iota \in \mathbb{N}}$ of integral manifolds of $d_{k}$ is an admissible sequence of integral manifolds of $d$ if there are a core distribution $e=\left(e_{0}, \ldots, e_{l}\right)$ of $d$, a Rolle leaf $W$ of $e$ and a definable family $\mathcal{B}$ of closed integral manifolds of $d_{k-l}$ such that each $V_{\iota}$ has core $W$ corresponding to $e$ and definable part in $\mathcal{B}$ corresponding to $W$. In this situation, we call $W$ the core of the sequence $\left(V_{\iota}\right)$ corresponding to $e$ and $\mathcal{B}$ a definable part of the sequence $\left(V_{\iota}\right)$ corresponding to $W$.

Remarks. (3) In the previous definition, we think of the core of $\left(V_{\iota}\right)$ as representing the "non-definable part" of $\left(V_{\iota}\right)$.

(4) Let $\left(V_{\iota}\right)$ be an admissible sequence of integral manifolds of $d$. Arguing as in Remark (2) above, we see that there is an admissible sequence $\left(U_{\iota}\right)$ of integral manifolds of $\left(d_{0}, \ldots, d_{k-1}\right)$ such that $V_{\iota} \subseteq U_{\iota}$ for $\iota \in \mathbb{N}$.

Definition 4.4. Let $\left(V_{\iota}\right)$ be an admissible sequence of integral manifolds of $d$. If $\left(V_{\iota}\right)$ converges to $K \in \mathcal{K}_{n}$, we call $K$ a pfaffian limit over $\mathcal{R}$. In this situation, we say that $K$ is obtained from $d$, and we put

$\operatorname{deg} K:=\min \{\operatorname{deg} f: K$ is obtained from $f\}$. 
Lemma 4.5. Let $K \subseteq \mathbb{R}^{n}$ be a pfaffian limit obtained from $d$. Then $K$ is definable in $\mathcal{P}(\mathcal{R})$ and $\operatorname{dim} K \leq \operatorname{dim} d$.

Proof. Let $\left(V_{\iota}\right)$ be an admissible sequence of integral manifolds of $d$ such that $K=\lim _{\iota} V_{\iota}$. Let $e, W$ and $\mathcal{B}$ be such that $W$ is the core of $\left(V_{\iota}\right)$ corresponding to $e$ and $\mathcal{B}$ is a definable part of $\left(V_{\iota}\right)$ corresponding to $W$. Since $W$ is definable in $\mathcal{P}(\mathcal{R})$, the family of all admissible integral manifolds of $d$ with core $W$ corresponding to $e$ and definable part in $\mathcal{B}$ corresponding to $W$ is definable in $\mathcal{P}(\mathcal{R})$. Hence the lemma follows from the versions of the Marker-Steinhorn theorem [17] found in [4, Theorem 3.1] and [14, Theorem 1].

For the remainder of this section, we let $e=\left(e_{0}, \ldots, e_{l}\right)$ be a core distribution of $d$ and $W$ be a Rolle leaf of $e$.

One reason for working with pfaffian limits over $\mathcal{R}$ is that they can be used to describe the frontiers of admissible integral manifolds. To see how this is done, we assume for Propositions 4.6 and 4.7 below that $M$ has a definable $C^{2}$-carpeting function $\phi$, and we adopt the corresponding notations introduced in Example 2.7. We assume that the Whitney stratification $\mathcal{C}$ is also compatible with $e$, and we let $N \in \mathcal{C}$ or $N=M^{\prime}$ and adopt here the corresponding notations introduced in Remark 4.2, By not requiring that all $W_{p}^{N}$ be distinct, we may assume that $q$ is independent of $N$. Thus, for $r>0$, $p=1, \ldots, q$ and every admissible integral manifold $V$ of $d$ with core $W$ and definable part $B$, each set $\phi^{-1}(r) \cap V_{p}^{N}$ is an admissible integral manifold of $d_{\phi}^{N}$ with core $W_{p}^{N}$ and definable part $\phi^{-1}(r) \cap B \cap N$.

Let now $V$ be an admissible integral manifold of $d$ with core $W$ and definable part $B$, and let $\left(r_{\kappa}\right)_{\kappa \in \mathbb{N}}$ be a sequence of positive real numbers such that $r_{\kappa} \rightarrow 0$ and $\lim _{\kappa}\left(\phi^{-1}\left(r_{\kappa}\right) \cap V\right)$ exists. Then for $p=1, \ldots, q$, the sequence $\left(\phi^{-1}\left(r_{\kappa}\right) \cap V_{p}^{N}\right)$ is an admissible sequence of integral manifolds of $d_{\phi}^{N}$ with core $W_{p}^{N}$ and definable part $\left\{\phi^{-1}(r) \cap B \cap N: r>0\right\}$. Passing to a subsequence, we may assume that $K_{p}^{N}(V):=\lim _{\kappa}\left(\phi^{-1}\left(r_{\kappa}\right) \cap V_{p}^{N}\right)$ exists for each $p$ and each $N$. Thus, each $K_{p}^{N}(V)$ is a pfaffian limit obtained from $d^{N}$.

Proposition 4.6. fr $V=K_{1}^{M^{\prime}}(V) \cup \cdots \cup K_{q}^{M^{\prime}}(V)$, and each $K_{p}^{M^{\prime}}(V)$ is a pfaffian limit over $\mathcal{R}$ such that $\operatorname{dim} K_{p}^{M^{\prime}}(V)<\operatorname{dim} d$ and $\operatorname{deg} K_{p}^{M^{\prime}}(V) \leq \operatorname{deg} d$.

Proof. Since $V$ is definable in $\mathcal{P}(\mathcal{R})$, we get from Lemma 1.6 that fr $V=$ $\lim _{\kappa}\left(\phi^{-1}\left(r_{\kappa}\right) \cap V\right)$. On the other hand, we have

$$
\bigcup_{p=1}^{q} K_{p}^{M^{\prime}}(V)=\bigcup_{C \in \mathcal{C}^{\prime}} \bigcup_{p=1}^{q} K_{p}^{N}(V),
$$

and both unions are contained in fr $V$. Thus, to finish our argument, we let $z \in \lim _{\kappa}\left(\phi^{-1}\left(r_{\kappa}\right) \cap V\right)$ and show that $z \in K_{p}^{N}(V)$ for some $N \in \mathcal{C}^{\prime}$ and 
$p \in\{1, \ldots, q\}$. Let $x_{\kappa} \in \phi^{-1}\left(r_{\kappa}\right) \cap V$ be such that $\lim x_{\kappa}=z$. Let $N \in \mathcal{C}$ be such that infinitely many $x_{\kappa}$ belong to $N$; passing to a subsequence, we may assume that $x_{\kappa} \in N$ for all $\kappa$. Then $N \in \mathcal{C}^{\prime}$ : otherwise, we have $g_{N} \cap d_{k} \subseteq d_{\phi}$, so the definability in $\mathcal{P}(\mathcal{R})$ implies that $\phi^{-1}(r) \cap V \cap N=\emptyset$ for all but finitely many $r$, which contradicts $\lim _{\kappa} \phi\left(x_{\kappa}\right)=0$. Thus, passing again to a subsequence, we may assume that there is a $p$ such that $x_{\kappa} \in \phi^{-1}\left(r_{\kappa}\right) \cap V_{p}^{N}$ for all $\kappa$. Hence $z \in K_{p}^{N}(V)$, as required.

Proposition 4.7. Let $\left(V_{\iota}\right)$ be an admissible sequence of integral manifolds of $d$ such that $K^{\prime}:=\lim _{\iota}$ fr $V_{\iota}$ exists. Then there are $q \in \mathbb{N}$ and pfaffian limits $K_{1}, \ldots, K_{q} \subseteq \mathbb{R}^{n}$ over $\mathcal{R}$ such that $K^{\prime}=K_{1} \cup \cdots \cup K_{q}$ and $\operatorname{dim} K_{p}<\operatorname{dim} d$ and $\operatorname{deg} K_{p} \leq \operatorname{deg} d$ for each $p$.

Proof. We may assume that $\left(V_{\iota}\right)$ has core $W$, and we adopt the notations introduced before Proposition 4.6, with $V_{\iota}$ in place of $V$ and $B\left(V_{\iota}\right) \in \mathcal{B}$, where $\mathcal{B}$ is a definable part of $\left(V_{\iota}\right)$. Passing to a subsequence if necessary, we may assume that $\lim _{\iota} K_{p}^{M^{\prime}}\left(V_{\iota}\right)$ exists for each $p$. Then by Proposition 4.6, we have

$$
K^{\prime}=\bigcup_{p=1}^{q} \lim _{\iota} K_{p}^{M^{\prime}}\left(V_{\iota}\right) .
$$

Hence $K^{\prime}=\bigcup_{p=1}^{q} \lim _{\iota}\left(\phi^{-1}\left(r_{\kappa(\iota)}\right) \cap\left(V_{\iota}\right)_{p}^{M^{\prime}}\right)$ for some subsequence $(\kappa(\iota))_{\iota}$. But each sequence $\left(\phi^{-1}\left(r_{\kappa(\iota)}\right) \cap\left(V_{\iota}\right)_{p}^{M^{\prime}}\right)$, is an admissible sequence of integral manifolds of $d_{\phi}^{M^{\prime}}$ with core $W_{p}^{M^{\prime}}$ and definable part $\left\{\phi^{-1}(r) \cap B \cap N: r>0, B \in \mathcal{B}\right\}$, as required.

Another reason for working with pfaffian limits over $\mathcal{R}$ is that they are themselves well behaved with respect to taking frontiers after intersecting with definable cells. To see this, we define the following distributions on $\mathbf{M}:=$ $M \times \mathbb{R}^{2}$, where we write $(x, r, \epsilon)$ for the typical element of $\mathbf{M}$ with $x \in M$ and $r, \epsilon \in \mathbb{R}$ : we set $\mathbf{d}_{0}:=g_{\mathbf{M}}, \mathbf{d}_{1}:=\operatorname{ker} d \epsilon \cap \mathbf{d}_{0}, \mathbf{d}_{2}:=\operatorname{ker} d r \cap \mathbf{d}_{1}$ and

$$
\begin{aligned}
\mathbf{d}_{2+j}(x, r, \epsilon) & :=\left(d_{j}(x) \times \mathbb{R}^{2}\right) \cap \mathbf{d}_{2}(x, r, \epsilon) & \text { for } j=1, \ldots, k, \\
\mathbf{e}_{j}(x, r, \epsilon) & :=e_{j}(x) \times \mathbb{R}^{2} & \text { for } j=0, \ldots, l .
\end{aligned}
$$

We also put $\mathbf{W}:=W \times \mathbb{R}^{2}$. Then $\mathbf{d}:=\left(\mathbf{d}_{0}, \ldots, \mathbf{d}_{2+k}\right)$ is a definable nested distribution on $\mathbf{M}$ with core distribution $\mathbf{e}:=\left(\mathbf{e}_{0}, \ldots, \mathbf{e}_{l}\right)$. Thus $\operatorname{deg} \mathbf{d} \leq \operatorname{deg} d$, and whenever $\left(V_{\iota}\right)$ is an admissible sequence of integral manifolds of $d$ with core $W$ and $\left(r_{\iota}, \epsilon_{\iota}\right) \in \mathbb{R}^{2}$ for $\iota \in \mathbb{N}$, the sequence $\left(V_{\iota} \times\left\{\left(r_{\iota}, \epsilon_{\iota}\right)\right\}\right)$ is an admissible sequence of integral manifolds of $\mathbf{d}$ with core $\mathbf{W}$.

Lemma 4.8. Let $K$ be a pfaffian limit obtained from $d$, and let $C \subseteq \mathbb{R}^{n}$ be a definable cell. Then there is a definable open subset $\mathbf{N}$ of $\mathbf{M}$ and there are 
$q \in \mathbb{N}$ and pfaffian limits $K_{1}, \ldots, K_{q}$ obtained from $\mathbf{d}^{\mathbf{N}}$ such that

$$
\operatorname{fr}(K \cap C)=\Pi_{n}\left(K_{1}\right) \cup \cdots \cup \Pi_{n}\left(K_{q}\right) ;
$$

in particular, $\operatorname{deg} K_{p} \leq \operatorname{deg} d$ for each $p$.

The following general observation is needed for the proof of this lemma:

Remark 4.9. In the situation of Remark 4.2, let $\left(V_{\iota}\right)$ be an admissible sequence of integral manifolds of $d$ with core $W$, and assume that $K^{N}:=$ $\lim _{\iota}\left(V_{\iota} \cap N\right)$ exists. After passing to a subsequence if necessary, we may assume that the sequence $\left(\left(V_{\iota}\right)_{p}^{N}\right)$, converges to a set $K_{p}^{N} \in \mathcal{K}_{n}$, for $p=1, \ldots, q$; then

$$
K^{N}=K_{1}^{N} \cup \cdots \cup K_{q}^{N} .
$$

Moreover, if $\mathcal{B}$ is the definable part of $\left(V_{\iota}\right)$, then each sequence $\left(\left(V_{\iota}\right)_{p}^{N}\right)$, is an admissible sequence of integral manifolds of $d^{N}$ with core $W_{p}^{N}$ corresponding to $e^{N}$ and definable part $\mathcal{B}^{N}:=\{B \cap N: B \in \mathcal{B}\}$. Thus, each $K_{p}^{N}$ is a pfaffian limit obtained from $d^{N}$; in particular, $\operatorname{deg} K_{p}^{N} \leq \operatorname{deg} d$.

Proof of Lemma 4.8. We let $\phi$ be a definable carpeting function on $C$ and put

$$
\mathbf{N}:=\left\{(x, r, \epsilon) \in \mathbf{M}: d\left(x, \phi^{-1}(r)\right)<\epsilon\right\},
$$

where we set $d(x, \emptyset):=\infty$ for all $x \in M$. Then $\mathbf{N}$ is an open, definable subset of $\mathbf{M}$, and since $K$ is compact and definable in $\mathcal{P}(\mathcal{R})$, we have $\operatorname{fr}(K \cap C)=$ $\lim _{r \rightarrow 0}\left(\phi^{-1}(r) \cap K\right)$ by Lemma 1.6. Moreover, we let $\left(V_{\iota}\right)$ be an admissible sequence of integral manifolds of $d$ such that $K=\lim _{\iota} V_{\iota}$; we may assume that $\left(V_{\iota}\right)$ has core $W$. Then for every $r>0$, the family of sets $\left\{\lim _{\iota}\left(V_{\iota} \cap N^{r, \epsilon}\right)\right.$ : $\epsilon>0\}$ is decreasing in $\epsilon$, where $\mathbf{N}^{r, \epsilon}:=\{x \in M:(x, r, \epsilon) \in \mathbf{N}\}$, so we have

$$
\phi^{-1}(r) \cap K=\lim _{\epsilon \rightarrow 0} \lim _{\iota}\left(V_{\iota} \cap \mathbf{N}^{r, \epsilon}\right) .
$$

Hence, after passing to a subsequence of $\left(V_{\iota}\right)$ if necessary, there are $r_{\iota} \rightarrow 0$ and $\epsilon_{\iota} \rightarrow 0$ such that

$$
\left.\operatorname{fr}(K \cap C)=\lim _{\iota}\left(V_{\iota} \cap \mathbf{N}^{r_{\iota}, \epsilon_{\iota}}\right)=\lim _{\iota} \Pi_{n}\left(\left(V_{\iota} \times\left\{r_{\iota}, \epsilon_{\iota}\right)\right\}\right) \cap \mathbf{N}\right) .
$$

Since $\lim _{\iota}\left(r_{\iota}, \epsilon_{\iota}\right)=(0,0)$, the right-hand side in the previous equality is equal to $\Pi_{n}\left(\lim _{\iota}\left(\left(V_{\iota} \times\left\{\left(r_{\iota}, \epsilon_{\iota}\right)\right\}\right) \cap \mathbf{N}\right)\right)$. Since the sequence $\left(V_{\iota} \times\left\{\left(r_{\iota}, \epsilon_{\iota}\right)\right\}\right)$ is an admissible sequence of integral manifolds of $\mathbf{d}$ with core $\mathbf{W}$, the lemma now follows from Remark 4.9 with $\mathbf{d}$ and $\mathbf{W}$ in place of $d$ and $W$.

One problem with the previous lemma is that $\operatorname{dim} \mathbf{d}=\operatorname{dim} d$, so it is possible that $\operatorname{dim} K_{p}>\operatorname{dim} \operatorname{fr}(K \cap C)$ for some $p$. To remedy this, we need a fiber cutting lemma for pfaffian limits over $\mathcal{R}$.

Definition 4.10. Let $K \subseteq \mathbb{R}^{n}$ be a pfaffian limit obtained from $d$. We say that $K$ is proper if $\operatorname{dim} K=\operatorname{dim} d$. 
Proposition 4.11. Let $K \subseteq \mathbb{R}^{n}$ be a pfaffian limit obtained from $d$ and $\nu \leq n$. Then there are $q \in \mathbb{N}$ and proper pfaffian limits $K_{1}, \ldots, K_{q} \subseteq \mathbb{R}^{n}$ over $\mathcal{R}$ such that

$$
\Pi_{\nu}(K)=\Pi_{\nu}\left(K_{1}\right) \cup \cdots \cup \Pi_{\nu}\left(K_{q}\right)
$$

and $\operatorname{deg} K_{p} \leq \operatorname{deg} d$ and $\operatorname{dim} K_{p}=\operatorname{dim} \Pi_{\nu}\left(K_{p}\right) \leq \operatorname{dim} K$ for each $p$.

The following remark is needed in the proof of this proposition and already appeared as Remark 3.5 in [15]; we restate it here for the convenience of the reader.

Remark 4.12. Let $S \subseteq \mathbb{R}^{k}$ be definable in an o-minimal expansion $\mathcal{S}$ of the real field and put $l:=\operatorname{dim} S$. Then there is a set $Y \subseteq S$, definable in $\mathcal{S}$, such that $S \subseteq \mathrm{cl} Y$, and for every $x \in Y$ there is a strictly increasing $\lambda:\{1, \ldots, l\} \longrightarrow\{1, \ldots, k\}$ such that $x$ is isolated in $S \cap \Pi_{\lambda}^{-1}\left(\Pi_{\lambda}(x)\right)$.

Proof of Proposition 4.11. We proceed by induction on $m=\operatorname{dim} M$; the case $m=0$ is trivial, so we assume that $m>0$ and that the proposition holds for lower values of $m$. Let $\left(V_{\iota}\right)$ be an admissible sequence of integral manifolds of $d$ such that $K=\lim _{\iota} V_{\iota}$. Choosing a suitable $C^{2}$-cell decomposition of $M$ compatible with $d$, and using Remark 4.9 and the inductive hypothesis, we reduce to the case where $M$ is a definable $C^{2}$-cell such that for every $s \leq \nu$ and every strictly increasing map $\lambda:\{1, \ldots, s\} \longrightarrow\{1, \ldots, \nu\}$, the rank of $\Pi_{\lambda}^{n}\left\lceil_{d_{l}(x)}\right.$ is constant for $x \in M$ and $l=1, \ldots, k$; for $l=k$ we denote this rank by $r_{\lambda}$. Putting $\Delta(\lambda):=\left\{d,\left(\operatorname{ker} d x_{\lambda(1)}\right)^{M}, \ldots,\left(\operatorname{ker} d x_{\lambda(s)}\right)^{M}\right\}$ as at the beginning of Section 3 and using the associated notation, this means that each $g \in \mathcal{D}_{\Delta(\lambda)}$ has dimension, and we let $d^{\lambda}:=d^{\Delta(\lambda), M}=\left(d_{0}^{\lambda}, \ldots, d_{k+r_{\lambda}}^{\lambda}\right)$ be the corresponding definable nested distribution on $M$ of dimension $m-k-r_{\lambda}$. It follows from the rank theorem and the fact that admissible integral manifolds of $d$ are closed in $M$ that $V_{\iota} \cap\left(\Pi_{\lambda}^{n}\right)^{-1}(y)$ is a closed integral manifold of $d_{k+r_{\lambda}}^{\lambda}$, for $\iota \in \mathbb{N}$ and $y \in \Pi_{\lambda}^{n}\left(V_{\iota}\right)$.

Let $s:=\operatorname{dim} \Pi_{\nu}(K)$; then $s \leq \operatorname{dim} d$ by Lemma 4.5, If $s=\operatorname{dim} d$, we are done, so we assume from now on that $s<\operatorname{dim} d$. Let $\lambda:\{1, \ldots, s\} \longrightarrow$ $\{1, \ldots, \nu\}$ be strictly increasing; since $s<\operatorname{dim} d$, we have

$$
\operatorname{dim} d^{\lambda} \geq \operatorname{dim} d-s>0
$$

in particular, $r_{\lambda}<\operatorname{dim} d$. Hence by Lemma 3.1 and because each fiber $V_{\iota} \cap$ $\left(\Pi_{\lambda}^{n}\right)^{-1}(y)$ is a closed submanifold of $M$, there is a closed, definable set $B_{\lambda} \subseteq M$ such that $\operatorname{dim} B_{\lambda}<m$ and for $y \in \mathbb{R}^{s}$ and $\iota \in \mathbb{N}$, each component of the fiber $V_{\iota} \cap\left(\Pi_{\lambda}^{n}\right)^{-1}(y)$ intersects the fiber $B_{\lambda} \cap\left(\Pi_{\lambda}^{n}\right)^{-1}(y)$.

In particular, $\Pi_{\lambda}^{n}\left(V_{\iota} \cap B_{\lambda}\right)=\Pi_{\lambda}^{n}\left(V_{\iota}\right)$ for all $\iota$, and for all $y \in \mathbb{R}^{s}$, every component of $\Pi_{\nu}\left(V_{\iota}\right) \cap\left(\Pi_{\lambda}^{\nu}\right)^{-1}(y)$ intersects the fiber $\Pi_{\nu}\left(V_{\iota} \cap B_{\lambda}\right) \cap\left(\Pi_{\lambda}^{\nu}\right)^{-1}(y)$.

We now denote by $\Lambda$ the set of all strictly increasing $\lambda:\{1, \ldots, s\} \longrightarrow$ $\{1, \ldots, \nu\}$. Passing to a subsequence if necessary, we may assume for $\lambda \in \Lambda$ 
that the sequence $\left(V_{\iota} \cap B_{\lambda}\right)_{\iota}$ converges to a compact set $K^{\lambda}$. Choosing a suitable $C^{2}$-cell decomposition of $B_{\lambda}$ and using again Remark 4.9, we see from the inductive hypothesis that the proposition holds with each $K^{\lambda}$ in place of $K$. It therefore remains to show that $\Pi_{\nu}(K)=\bigcup_{\lambda \in \Lambda} \Pi_{\nu}\left(K^{\lambda}\right)$. To see this, we fix a $\lambda \in \Lambda$; since each $\Pi_{\nu}\left(K^{\lambda}\right)$ is closed, it suffices by Remark 4.12 to establish the following

Claim. Let $y \in \Pi_{\lambda}^{n}(K)$, and let $x \in \Pi_{\nu}(K) \cap\left(\Pi_{\lambda}^{\nu}\right)^{-1}(y)$ be isolated. Then $x \in \Pi_{\nu}\left(K^{\lambda}\right)$.

To see this, note that $\Pi_{\nu}(K)=\lim _{\iota} \Pi_{\nu}\left(V_{\iota}\right)$ since $M$ is bounded. Let $x_{\iota} \in$ $\Pi_{\nu}\left(V_{\iota}\right)$ be such that $\lim _{\iota} x_{\iota}=x$, and put $y_{\iota}:=\Pi_{\lambda}^{\nu}\left(x_{\iota}\right)$. Let $C_{\iota} \subseteq \mathbb{R}^{\nu}$ be the component of $\Pi_{\nu}\left(V_{\iota}\right) \cap\left(\Pi_{\lambda}^{\nu}\right)^{-1}\left(y_{\iota}\right)$ containing $x_{\iota}$, and let $x_{\iota}^{\prime}$ belong to $C_{\iota} \cap \Pi_{\nu}\left(V_{\iota} \cap B_{\lambda}\right)$. Since also $\Pi_{\nu}\left(K^{\lambda}\right)=\lim _{\iota} \Pi_{\nu}\left(V_{\iota} \cap B_{\lambda}\right)$, we may assume, after passing to a subsequence if necessary, that $x^{\prime}:=\lim _{\iota} x_{\iota}^{\prime} \in \Pi_{\nu}\left(K^{\lambda}\right)$. We show that $x^{\prime}=x$, which then proves the claim. Assume for a contradiction that $x^{\prime} \neq x$, and let $\delta>0$ be such that $\delta \leq\left|x-x^{\prime}\right|$ and

$$
B(x, \delta) \cap \Pi_{\nu}(K) \cap\left(\Pi_{\lambda}^{\nu}\right)^{-1}(y)=\{x\} .
$$

Then for all sufficiently large $\iota$, there is an $x_{\iota}^{\prime \prime} \in C_{\iota}$ such that $\delta / 3 \leq\left|x_{\iota}^{\prime \prime}-x_{\iota}\right| \leq$ $2 \delta / 3$, because $x_{\iota}, x_{\iota}^{\prime} \in C_{\iota}$ and $C_{\iota}$ is connected. Passing to a subsequence if necessary, we may assume that $x^{\prime \prime}:=\lim _{\iota} x_{\iota}^{\prime \prime} \in \Pi_{\nu}(K)$. Then $x^{\prime \prime} \in B(x, \delta)$ with $x^{\prime \prime} \neq x$, and since $x_{\iota}^{\prime \prime} \in C_{\iota}$ implies that $\Pi_{\lambda}^{\nu}\left(x_{\iota}^{\prime \prime}\right)=y_{\iota}$, we get $\Pi_{\lambda}^{\nu}\left(x^{\prime \prime}\right)=y$, contradicting (4.1).

Combining Lemma 4.8 with Proposition 4.11 gives:

Corollary 4.13. Let $K$ be a pfaffian limit obtained from $d$, and let $C \subseteq \mathbb{R}^{n}$ be a definable cell. Then there are $q \in \mathbb{N}$ and proper pfaffian limits $K_{1}, \ldots, K_{q} \subseteq$ $\mathbb{R}^{n+2}$ over $\mathcal{R}$ such that

$$
\operatorname{fr}(K \cap C)=\Pi_{n}\left(K_{1}\right) \cup \cdots \cup \Pi_{n}\left(K_{q}\right),
$$

and $\operatorname{deg} K_{p} \leq \operatorname{deg} d$ and $\operatorname{dim} K_{p}<\operatorname{dim}(K \cap C)$ for each $p$.

\section{BLOWING-UP ALONG A NESTED DISTRIBUTION}

In this section, we establish a criterion for generic portions (in the sense of dimension and degree) of pfaffian limits over $\mathcal{R}$ to be integral manifolds of definable nested distributions. We fix a bounded, definable manifold $M \subseteq \mathbb{R}^{n}$ of dimension $m$ and a definable nested distribution $d=\left(d_{0}, \ldots, d_{k}\right)$ on $M$, and we assume both are of class $C^{2}$. 
Definition 5.1. Put $n_{1}:=n+n^{2}$ and let $\Pi: \mathbb{R}^{n_{1}} \longrightarrow \mathbb{R}^{n}$ denote the projection on the first $n$ coordinates. We define

$$
\begin{aligned}
M^{1} & :=\operatorname{gr} d_{k} \subseteq M \times G_{n}^{m-k} \subseteq \mathbb{R}^{n_{1}}, \text { the graph of the distribution } d_{k}, \\
d_{l}^{1} & :=\left(\Pi \uparrow_{M^{1}}\right)^{*} d_{l}, \text { the pull-back to } M^{1} \text { of } d_{l} \text { via } \Pi \text {, for } l=0, \ldots, k .
\end{aligned}
$$

We call $d^{1}:=\left(d_{0}^{1}, \ldots, d_{k}^{1}\right)$ the blowing-up of $d$ (along $d_{k}$ ); note that $M^{1}$ is of class $C^{2}$, while $d^{1}$ is of class $C^{1}$. Finally, for $l=0, \ldots, k$ and an integral manifold $V$ of $d_{l}$, we define

$$
\left.V^{1}:=(\Pi\rceil_{M^{1}}\right)^{-1}(V),
$$

the lifting of $V$ (along $d_{k}$ ). Note that, in this situation, $V^{1}$ is an integral manifold of $d_{l}^{1}$, and if $l=k$, then $V^{1}$ is also the graph of the Gauss map $g_{V}$.

Next, we write $M=\bigcup M_{\sigma}$, where $\sigma$ ranges over $\Sigma_{n}$ and the $M_{\sigma}:=M_{\sigma, 2}$ are as before Lemma 1.3 with $d$ and $\eta$ there equal to $d_{k}$ and 2 here.

Definition 5.2. For an integral manifold $V$ of $d_{k}$ and $\sigma \in \Sigma_{n}$, we put $V_{\sigma}:=$ $V \cap M_{\sigma}$. Then $V_{\sigma}$ is an integral manifold of $d_{k}$, and we define

$$
F^{1} V:=\bigcup_{\sigma \in \Sigma_{n}} \text { fr } V_{\sigma}^{1}
$$

For our criterion, we let $D \subseteq \operatorname{cl} M^{1}$ be a definable $C^{2}$-cell such that $C:=$ $\Pi(D)$ has the same dimension as $D$ and $C$ is compatible with $M_{\sigma}$ and fr $M_{\sigma}$ for every $\sigma \in \Sigma_{n}$. Then $D=\operatorname{gr} g$, where $g: C \longrightarrow G_{n}^{m-k}$ is a definable map, and we assume that the following hold:

(i) the map $g \cap g_{C}$ has dimension and hence is a distribution on $C$;

(ii) if $g=g \cap g_{C}$, then either $g$ is integrable or $g$ is nowhere integrable.

We also assume that there is a definable set $W \subseteq \operatorname{cl} M^{1}$ such that $W \cap D=\emptyset$ and both $W$ and $W \cup D$ are open in $\mathrm{cl}^{1}$. In this situation, for any sequence $\left(V_{\iota}\right)$ of integral manifolds of $d_{k}$ such that $K:=\lim _{\iota} V_{\iota}^{1}$ and $K^{\prime}:=\lim _{\iota} F^{1} V_{\iota}$ exist, we put

$$
L_{\left(V_{\iota}\right)}:=(D \cap K) \backslash\left(K^{\prime} \cup \operatorname{fr}(W \cap K)\right) .
$$

Remark. Assume that $\left(V_{\iota}\right)$ is an admissible sequence of integral manifolds of $d$ such that $K:=\lim _{\iota} V_{\iota}^{1}$ and $K^{\prime}:=\lim _{\iota} F^{1} V_{\iota}$ exist, and assume that $K$ is proper. Then $L_{\left(V_{\iota}\right)}$ is a generic subset of $K$ in the following sense: $\left(V_{\iota}^{1}\right)$ is an admissible sequence of integral manifolds of $d^{1}$, and each $\left(V_{\iota}\right)_{\sigma}^{1}$ is a finite union of admissible integral manifolds of $\left(d^{1}\right)^{M_{\sigma}}$. By Lemma 4.5, Proposition 4.7 and Remark 4.9, $K^{\prime}$ is a finite union of pfaffian limits over $\mathcal{R}$ of dimension less than $\operatorname{dim} K$ and degree at most $\operatorname{deg} d$. Moreover, by cell decomposition and Corollary 4.13, there is a finite union $F \subseteq \mathbb{R}^{n_{1}+2}$ of pfaffian limits over $\mathcal{R}$ of dimension less than $\operatorname{dim} K$ and degree at most $\operatorname{deg} d$ such that $\operatorname{fr}(W \cap K)=$ $\Pi_{n_{1}}(F)$. 
Finally, we let $g^{1}: D \longrightarrow G_{n_{1}}^{m-k}$ be the pull-back of $g \cap g_{C}$ to $D$ via $\Pi \uparrow_{D}$.

Proposition 5.3. In this situation, exactly one of the following holds:

(1) $L_{\left(V_{\iota}\right)}=\emptyset$ for every admissible sequence $\left(V_{\iota}\right)$ of integral manifolds of $d$ such that $\lim _{\iota} V_{\iota}^{1}$ and $\lim _{\iota} F^{1} V_{\iota}$ exist;

(2) $g$ is an integrable distribution on $C$, and for every admissible sequence $\left(V_{\iota}\right)$ of integral manifolds of $d$ such that $\lim _{\iota} V_{\iota}^{1}$ and $\lim _{\iota} F^{1} V_{\iota}$ exist, the set $L_{\left(V_{\iota}\right)}$ is an embedded integral manifold of $g^{1}$ and an open subset of $\lim _{\iota} V_{\iota}^{1}$.

In particular, if $D$ is an open subset of $M^{1}$ and $\left(V_{\iota}\right)$ is an admissible sequence of integral manifolds of $d$ such that $\lim _{\iota} V_{\iota}^{1}$ exists, then $D \cap \lim _{\iota} V_{\iota}^{1}$ is a finite union of leaves of $\left(d_{k}^{1}\right)^{D}$.

We need the following observation for the proof of this proposition:

Remark. Let $\sigma \in \Sigma_{n}$. Then $\sigma$ induces a diffeomorphism $\sigma: G_{n} \longrightarrow G_{n}$ defined, in the notation of our conventions, by $\sigma(y):=A_{\sigma(\operatorname{ker} y)}$, and we define $\sigma^{1}: \mathbb{R}^{n} \times G_{n} \longrightarrow \mathbb{R}^{n} \times G_{n}$ by $\sigma^{1}(x, y):=(\sigma(x), \sigma(y))$. Note that $\sigma^{1}$ is also a permutation of coordinates. The map $g^{\sigma}: \sigma(C) \longrightarrow G_{n}^{m-k}$ defined by $g^{\sigma}(\sigma(x)):=\sigma(g(x))$ satisfies $\left(g^{\sigma}\right)^{1}=\sigma^{1} \circ g^{1} \circ\left(\sigma^{1}\right)^{-1}$. Moreover, if $\left(V_{\iota}\right)$ is a sequence of integral manifolds of $d_{k}$ such that $\lim _{\iota} V_{\iota}^{1}$ exists, then $\lim _{\iota} \sigma\left(V_{\iota}^{1}\right)$ also exists and $\sigma^{1}(D) \cap \lim _{\iota} \sigma^{1}\left(V_{\iota}^{1}\right)=\sigma^{1}\left(D \cap \lim _{\iota} V_{\iota}^{1}\right)$.

Proof of Proposition 5.3. By the previous remark and Remark 4.2, after replacing $M$ by $\sigma\left(M_{\sigma}\right)$ and $W$ by $\sigma^{1}\left(W \cap \mathrm{cl} M_{\sigma}^{1}\right)$ for every $\sigma \in \Sigma_{n}$ satisfying $C \subseteq \mathrm{cl} M_{\sigma}$, we may assume for the rest of this proof that $d_{k}$ is 2-bounded and prove the proposition with fr $V_{\iota}^{1}$ in place of $F^{1}\left(V_{\iota}\right)$. Thus, we let $\left(V_{\iota}\right)$ be an admissible sequence of integral manifolds of $d$ such that $K:=\lim _{\iota} V_{\iota}^{1}$ and $K^{\prime}:=\lim _{\iota}$ fr $V_{\iota}^{1}$ exist, and we put

$$
L:=(D \cap K) \backslash\left(K^{\prime} \cup \operatorname{fr}(W \cap K)\right) .
$$

For the remainder of this proof, we simply write "lim" in place of "lim,". By definition of admissible sequence of integral manifolds and Corollary 3.3)(2), there is a $\nu \in \mathbb{N}$ such that for every open box $U \subseteq \mathbb{R}^{n}$ and every $\iota$, the set $U \cap V_{\iota}$ has at most $\nu$ components. We assume that $L \neq \emptyset$; we need to show that $g$ is an integrable distribution on $C$ and that $L$ is an embedded integral manifold of $g^{1}$ and an open subset of $K$.

To do so, we choose an arbitrary $(x, y) \in L$ with $x \in \mathbb{R}^{n}$ and $y \in G_{n}$. Since $W \cup D$ is open in cl $M^{1}$, there is a bounded open box $B \subseteq \mathbb{R}^{n_{1}}$ such that $(x, y) \in B$ and

$$
\text { cl } B \cap K \subseteq D \backslash\left(K^{\prime} \cup \operatorname{fr}(W \cap K)\right) \text {; }
$$


in particular, $(x, y) \in D$. We write $B=B_{0} \times B_{1}$ with $B_{0} \subseteq \mathbb{R}^{n}$ and $B_{1} \subseteq \mathbb{R}^{n^{2}}$. Since $D$ is the graph of the continuous map $g$ and $C$ is locally closed, we may also assume, after shrinking $B_{0}$ if necessary, that $D \cap\left(\operatorname{cl} B_{0} \times \operatorname{fr} B_{1}\right)=\emptyset$.

On the other hand, after passing to a subsequence if necessary, we may assume that $\lim \left(B \cap V_{\iota}^{1}\right), \lim V_{\iota, B}^{1}$ and $\lim$ fr $V_{\iota, B}$ exist, where $V_{\iota, B}:=\left\{x \in V_{\iota}\right.$ : $\left.\left(x, T_{x} V_{\iota}\right) \in B\right\}$. Then

$$
B \cap K=B \cap \lim \left(B \cap V_{\iota}^{1}\right)=B \cap \lim V_{\iota, B}^{1} .
$$

We now claim that $x \notin \lim \operatorname{fr} V_{\iota, B}$ : in fact, since $\operatorname{fr} V_{\iota}^{1} \cap \operatorname{cl} B=\emptyset$ for all sufficiently large $\iota$, we have $\operatorname{fr} V_{\iota, B}^{1} \subseteq$ fr $B$ for all sufficiently large $\iota$. Also, $\lim V_{\iota, B}^{1} \subseteq \mathrm{cl} B \cap \lim V_{\iota}^{1}$ is disjoint from $\mathrm{cl} B_{0} \times$ fr $B_{1}$ by the previous paragraph, so cl $V_{\iota, B}^{1}$ is disjoint from cl $B_{0} \times$ fr $B_{1}$ for all sufficiently large $\iota$. Hence fr $V_{\iota, B}^{1} \subseteq$ fr $B_{0} \times B_{1}$ for all sufficiently large $\iota$. Since $B$ is bounded, we also have that fr $V_{\iota, B} \subseteq \Pi_{n}$ (fr $\left.V_{\iota, B}^{1}\right)$, and it follows that fr $V_{\iota, B} \subseteq$ fr $B_{0}$ for all sufficiently large $\iota$, which proves the claim.

Since each $V_{\iota}$ is a closed submanifold of $M$, we now apply Lemma 1.5 with $V_{\iota, B}$ in place of $V_{\iota}$ and $\eta=2$, to obtain a corresponding open neighbourhood $U \subseteq B_{0}$ of $x$ and $f_{1}, \ldots, f_{\nu}: \Pi_{m-k}(U) \longrightarrow \mathbb{R}^{n-m+k}$. We let $\lambda \in\{1, \ldots, \nu\}$ be such that $x \in \operatorname{gr} f_{\lambda}$. We claim that for every $x^{\prime} \in \operatorname{gr} f_{\lambda} \cap U$, the map $f_{\lambda}$ is differentiable at $z^{\prime}:=\Pi_{m-k}\left(x^{\prime}\right)$ with $T_{x^{\prime}}$ gr $f_{\lambda}=g\left(x^{\prime}\right)$; since $x^{\prime}$ is arbitrary, this claim implies that $\operatorname{gr} f_{\lambda}$ is an embedded, connected integral manifold of g. Assumption (ii) and Remark 2.4(6) then imply that $g$ is an integrable distribution on $C$. Since $(x, y) \in L$ was arbitrary, it follows that $L$ is an embedded integral manifold of $g^{1}$, as desired.

To prove the claim, let $f_{\lambda, \iota}: \Pi_{m-k}(U) \longrightarrow \mathbb{R}^{n-m+k}$ be the functions corresponding to $f_{\lambda}$ as in the proof of Lemma 1.5. After a linear change of coordinates if necessary, we may assume that $g\left(x^{\prime}\right)=\mathbb{R}^{m-k} \times\{0\}$ (the subspace spanned by the first $m-k$ coordinates). It now suffices to show that $f_{\lambda}$ is $\eta$-Lipschitz at $x^{\prime}$ for every $\eta>0$, since then $T_{x^{\prime}}$ gr $f_{\lambda}=\mathbb{R}^{m-k} \times\{0\}$. So let $\eta>0$; since $\lim V_{\iota, B}^{1} \subseteq D=\operatorname{gr} g$ and $x^{\prime} \in C$, and since $C$ is locally closed and $g$ is continuous, there is a neighborhood $U^{\prime} \subseteq U$ of $x^{\prime}$ such that gr $f_{\lambda, \iota} \cap U^{\prime}$ is $\frac{\eta}{m-k}$-bounded for all sufficiently large $\iota$. Thus by Lemma 1.5 again, $f_{\lambda}$ is $\eta$-Lipschitz at $x^{\prime}$, as required.

Finally, if $D$ is open in $M^{1}$, then $g=d_{k} \Gamma_{C}$ and we can take $W:=\emptyset$. Since $C$ is open in $M$ and compatible with $M_{\sigma}$ and fr $M_{\sigma}$ for $\sigma \in \Sigma_{n}$, we have $C \cap$ fr $M_{\sigma}=\emptyset$ for each $\sigma$. Hence $F^{1}\left(V_{\iota}\right) \cap D=\emptyset$, and it follows that $L_{\left(V_{\iota}\right)}=D \cap \lim V_{\iota}$ in this case.

\section{LifTing A Distribution to A NESTED DistRIBUtion}

In the proofs of Propositions 7.1 and 10.4 below, we will encounter individual distributions, such as the distribution $g$ in the previous section, that need to 
be lifted to a given nested distribution to produce a new nested distribution of lower dimension. More precisely, we will encounter the following situation: we are given $p \in \mathbb{N} \cup\{\infty, \omega\}$ such that $p \geq 2$, a definable $C^{p}$-cell $N \subseteq \mathbb{R}^{m}$ with $m \geq n$ and a definable, integrable nested $C^{p}$-distribution $f=\left(f_{0}, \ldots, f_{l}\right)$ on $N$. We are also given a definable $C^{p}$-cell $D \subseteq \mathbb{R}^{n}$ such that $\Pi_{n}(N) \subseteq D$, a $k \leq \operatorname{dim} D$ and a definable, integrable distribution $h: D \longrightarrow G_{n}^{k}$ on $D$ of class $C^{p}$. We let $\nu \leq n$, and we assume that for all $\mu \leq \nu$ and all strictly increasing $\lambda:\{1, \ldots, \mu\} \longrightarrow\{1, \ldots, \nu\}$ the dimension of the spaces

$$
F_{\lambda}(y):=\Pi_{\lambda}\left(f_{l}(y)\right) \quad \text { and } \quad F_{\lambda}^{h}(y):=\Pi_{\lambda}\left(\Pi_{\nu}\left(f_{l}(y)\right) \cap \Pi_{\nu}\left(h\left(\Pi_{n}(y)\right)\right)\right)
$$

is constant as $y$ ranges over $N$; we denote these dimensions below by $\operatorname{dim} F_{\lambda}$ and $\operatorname{dim} F_{\lambda}^{h}$, respectively. For the identity map $\lambda:\{1, \ldots, \nu\} \longrightarrow\{1, \ldots, \nu\}$, we set $F:=F_{\lambda}$ and $F^{h}:=F_{\lambda}^{h}$ and put $\mu:=\operatorname{dim} F^{h} \leq k$. Finally, we assume that $\operatorname{dim} F \geq \mu+1$.

Remark and Definition 6.1. The assumptions in the previous paragraph imply that there is a strictly increasing $\lambda:\{1, \ldots, \mu+1\} \longrightarrow\{1, \ldots, \nu\}$ such that $\operatorname{dim} F_{\lambda}=\mu+1$ and $\operatorname{dim}\left(F_{\lambda}^{h}\right)=\mu$. We let $f_{l+1}: N \longrightarrow G_{m}$ be the map on $N$ defined by

$$
f_{l+1}(y):=f_{l}(y) \cap\left(\Pi_{\lambda}^{m}\right)^{-1}\left(F_{\lambda}^{h}(y)\right) .
$$

The map $f_{l+1}$ is definable, $\operatorname{dim} f_{l+1}(y)=\operatorname{dim} f_{l}(y)-1$ and $\operatorname{dim} \Pi_{\nu}\left(f_{l+1}(y)\right)=$ $\operatorname{dim} F-1$ for $y \in N$; in particular, $f^{\prime}:=\left(f_{0}, \ldots, f_{l+1}\right)$ is a definable nested distribution on $N$.

Next, let $Z \subseteq N$ be an embedded integral manifold of $f_{l}$, and let $L \subseteq D$ be an embedded integral manifold of $h$. By our assumption on $f_{l}, \Pi_{\nu} \Upsilon_{Z}$ has constant rank $\operatorname{dim} F$; we assume here in addition that $\Pi_{\nu}(Z)$ is a submanifold of $\mathbb{R}^{\nu}$. Similarly, $\Pi_{\nu} \uparrow_{L}$ has constant rank, and we assume here in addition that $\Pi_{\nu}(L)$ is a submanifold of $\mathbb{R}^{\nu}$. Then by our assumptions and the Rank Theorem, $\Pi_{\nu}(Z) \cap \Pi_{\nu}(L)$ is either empty or a submanifold of $\Pi_{\nu}(D)$ of dimension $\mu$. Let also $L^{\prime} \subseteq \Pi_{\nu}(Z) \cap \Pi_{\nu}(L)$ be a submanifold of dimension $\mu$. Again by our assumption on $\left.F_{\lambda}^{h}, \Pi_{\lambda}^{\nu}\right\rceil_{L^{\prime}}$ is an immersion; we also assume here that $\Pi_{\lambda}^{\nu}\left(L^{\prime}\right)$ is a submanifold of $\mathbb{R}^{\mu+1}$. In this situation, we define

$$
Z\left(L^{\prime}\right):=Z \cap\left(\Pi_{\lambda}^{m}\right)^{-1}\left(\Pi_{\lambda}^{\nu}\left(L^{\prime}\right)\right) .
$$

Then by the Rank Theorem, the set $Z\left(L^{\prime}\right)$ is an integral manifold of $f_{l+1}$.

Lemma 6.2. The nested distribution $f^{\prime}$ is integrable.

Proof. The integrability of $f_{l}$ and $h$ and our assumptions imply that for $y \in N$, there are an integral manifold $Z$ of $f_{l}$ containing $y$ and an integral manifold $L$ of $h$ containing $\Pi_{n}(y)$. By the Rank Theorem, after shrinking $Z$ and $L$ if necessary, we may assume that $\Pi_{\nu}(Z)$ and $\Pi_{\nu}(L)$ are submanifolds of $\mathbb{R}^{\nu}$, so that $L^{\prime}:=\Pi_{\nu}(Z) \cap \Pi_{\nu}(L)$ is a nonempty submanifold of $\mathbb{R}^{\nu}$. Shrinking $Z$ and 
$L$ again if necessary, we may also assume by the Rank Theorem that $\Pi_{\lambda}\left(L^{\prime}\right)$ is a submanifold of $\mathbb{R}^{\mu+1}$. So by Remark 6.1, the corresponding $Z\left(L^{\prime}\right)$ is an integral manifold of $f_{l+1}$ containing $y$.

For the next proposition, we let $\mathcal{S}$ be an o-minimal expansion of $\mathcal{R}$.

Proposition 6.3. Let $Z$ be an integral manifold of $f_{l}$ and $L$ be an integral manifold of $h$, and assume that both $Z$ and $L$ are definable in $\mathcal{S}$ and $\Pi_{n}(Z) \cap$ $L \neq \emptyset$. Then there are integral manifolds $Z_{1}^{\prime}, \ldots, Z_{q}^{\prime}$ of $f_{l+1}$ contained in $Z$ and definable in $\mathcal{S}$ such that $\Pi_{n}(Z) \cap L \subseteq \Pi_{n}\left(Z_{1}^{\prime}\right) \cup \cdots \cup \Pi_{n}\left(Z_{q}^{\prime}\right)$ and $\operatorname{dim} \Pi_{\nu}\left(Z_{p}^{\prime}\right)<\operatorname{dim} \Pi_{\nu}(Z)$ for each $p$.

Proof. By Lemma 1.1 with $\mathcal{S}$ in place of $\mathcal{R}$, we may assume that $\Pi_{\nu}(Z)$ and $\Pi_{\nu}(L)$ are submanifolds of $\mathbb{R}^{\nu}$. By the above and Lemma 1.1, we have $\Pi_{\nu}(Z) \cap$ $\Pi_{\nu}(L)=L_{1}^{\prime} \cup \cdots \cup L_{q}^{\prime}$, where each $L_{p}^{\prime}$ is an open subset of $\Pi_{\nu}(Z) \cap \Pi_{\nu}(L)$ such that $\Pi_{\lambda}\left(L_{p}^{\prime}\right)$ is a submanifold of $\mathbb{R}^{\mu+1}$. Now we take $Z_{p}^{\prime}:=Z\left(L_{p}^{\prime}\right)$, and we claim that these $Z_{p}^{\prime}$ work. To see this, we let $x \in \Pi_{n}(Z) \cap L$ and let $p \in\{1, \ldots q\}$ be such that $\Pi_{\nu}(x) \in L_{p}^{\prime}$; we show that $x \in \Pi_{n}\left(Z_{p}^{\prime}\right)$. Since $Z \cap\left(\Pi_{\lambda}^{m}\right)^{-1}\left(\Pi_{\lambda}(x)\right) \subseteq Z_{p}^{\prime}$ by definition, and since $\left(\Pi_{n}^{m}\right)^{-1}(x) \subseteq\left(\Pi_{\lambda}^{m}\right)^{-1}\left(\Pi_{\lambda}(x)\right)$, it follows that $Z \cap\left(\Pi_{n}^{m}\right)^{-1}(x) \subseteq Z_{p}^{\prime}$, that is, $x \in \Pi_{n}\left(Z_{p}^{\prime}\right)$. The proposition now follows from Remark and Definition 6.1.

\section{REWRITING PFAFFIAN LIMITS}

The goal of this section is to prove Proposition 1 of the introduction using Corollary 4.13 and

Proposition 7.1. Let $K \subseteq \mathbb{R}^{n}$ be a pfaffian limit over $\mathcal{R}$. Then there is a $q \in \mathbb{N}$ and, for $p=1, \ldots, q$, there are $n_{p} \geq n$ and an integral manifold $U_{p} \subseteq \mathbb{R}^{n_{p}}$ over $\mathcal{R}$ such that $K \subseteq \Pi_{n}\left(U_{1}\right) \cup \cdots \cup \Pi_{n}\left(U_{q}\right)$ and for each $p$, the set $U_{p}$ is definable in $\mathcal{P}(\mathcal{R})$ and $\operatorname{dim} \Pi_{n}\left(U_{p}\right) \leq \operatorname{dim} K$.

Proof. Let $M \subseteq \mathbb{R}^{n}$ be a definable $C^{2}$-manifold of dimension $m, d=\left(d_{0}, \ldots, d_{k}\right)$ a nested distribution on $M$ and $\left(V_{\iota}\right)$ an admissible sequence of integral manifolds of $d$ such that $K=\lim _{\iota} V_{\iota}$. We proceed by induction on the pair $(\operatorname{deg} d, \operatorname{dim} K)$, where we consider $\mathbb{N}^{2}$ with its lexicographic ordering. If $\operatorname{deg} d=$ 0 , then $K$ is definable in $\mathcal{R}$ by [4, Theorem 3.1] or [14, Theorem 1], so the proposition follows from cell decomposition and Example 2. If $\operatorname{dim} K=0$, then $K$ is finite and the proposition follows again from Example 2. Therefore, we assume that $\operatorname{deg} d>0$ and $\operatorname{dim} K>0$ and that the proposition holds for lower values of $(\operatorname{deg} d, \operatorname{dim} K)$. Moreover, by Proposition 4.11, we may assume that $K$ is a proper pfaffian limit over $\mathcal{R}$, that is, $\operatorname{dim} K=\operatorname{dim} d=m-k$. By $C^{m+2}$-cell decomposition, Remark 4.9 and the inductive hypothesis, we may assume that $M$ and $d$ are of class $C^{m+2}$. If $\sim_{d_{k}}$ is definable, then we are done 
as in the case $\operatorname{deg} d=0$. We therefore assume from now on that $\sim_{d_{k}}$ is not definable.

We now blow up $m+1$ times along $d_{k}$, that is, we put $n_{0}:=n, M^{0}:=M$ and $d^{0}:=d$, and we put $V^{0}:=V$ and $F^{0} V:=\bigcup_{\sigma \in \Sigma_{n}}$ fr $V_{\sigma}$ for every integral manifold $V$ of $d_{l}$ with $l \in\{0, \ldots, k\}$. By induction on $j=1, \ldots, m+1$ we define $n_{j}:=\left(n_{j-1}\right)_{1}=n_{j-1}+n_{j-1}^{2}, M^{j}:=\left(M^{j-1}\right)^{1}=\operatorname{gr} d_{k}^{j-1}$ and $d^{j}:=\left(d^{j-1}\right)^{1}$, and we define the corresponding liftings $V^{j}:=\left(V^{j-1}\right)^{1}$ and $F^{j} V:=F^{1} V^{j-1}$ for every integral manifold $V$ of $d_{l}$ with $l \in\{0, \ldots, k\}$. For $0 \leq i \leq j \leq m+1$, we also let $\pi_{i}^{j}: \mathbb{R}^{n_{j}} \longrightarrow \mathbb{R}^{n_{i}}$ be the projection on the first $n_{i}$ coordinates.

Passing to a subsequence if necessary, we may assume that $K^{j}:=\lim _{\iota} V_{\iota}^{j}$ and $\lim _{\iota} F^{j} V_{\iota}$ exist for $j=0, \ldots, m$ (so $K^{0}=K$ ). Then $\pi_{0}^{j}\left(K^{j}\right)=K$ for each $j$, and since $K$ is proper, each $K^{j}$ is also proper. It follows from Remark 4.9, Corollary 4.7 and the inductive hypothesis that

(I) the proposition holds with each $\lim _{\iota} F^{j} V_{\iota}$ in place of $K$.

For $j=0, \ldots, m+1$, we set $M_{\sigma}^{j}:=\left(M^{j}\right)_{\sigma, 2}$ as in Lemma 1.3 with $M, d$ and $\eta$ there equal to $M^{j}, d_{k}^{j}$ and 2 here. We let $\mathcal{C}^{j}$ be a $C^{2}$-cell decomposition of $\mathbb{R}^{n_{j}}$ compatible with $M^{j}$, fr $M^{j},\left\{M_{\sigma}^{j}: \sigma \in \Sigma_{n_{j}}\right\}$ and $\left\{\right.$ fr $\left.M_{\sigma}^{j}: \sigma \in \Sigma_{n_{j}}\right\}$, and we put

$$
\mathcal{C}_{M}^{j}:=\left\{C \in \mathcal{C}^{j}: C \subseteq \mathrm{cl} M^{j}\right\} .
$$

Refining each $\mathcal{C}^{j}$ in order of decreasing $j \in\{0, \ldots, m\}$ if necessary, we may assume for each such $j$ that

(i) $\mathcal{C}^{j}$ is a stratification compatible with $\left\{\pi_{j}^{j+1}(C): C \in \mathcal{C}^{j+1}\right\}$;

and for every $D \in \mathcal{C}_{M}^{j+1}$ that is the graph of a map $g: C \longrightarrow G_{n_{j}}^{m-k}$, where $C:=\pi_{j}^{j+1}(D)$, that

(ii) the map $g \cap g_{C}$ has dimension and hence is a distribution on $C$;

(iii) if $g=g \cap g_{C}$, then either $g$ is integrable or $g$ is nowhere integrable.

By Corollary 4.13 and the inductive hypothesis,

(II) for $j=0, \ldots, m$ and $E \in \mathcal{C}_{M}^{j}$, the proposition holds with $\operatorname{fr}\left(K^{j} \cap E\right)$ in place of $K$.

We now fix $j \in\{0, \ldots, m\}$ and a cell $C \in \mathcal{C}_{M}^{j}$ such that $\operatorname{dim} C \geq j$.

Claim 1: There is a $q \in \mathbb{N}$ and, for $p=1, \ldots, q$, there are $m_{p} \geq n_{j}$ and an integral manifold $U_{p} \subseteq \mathbb{R}^{m_{p}}$ over $\mathcal{R}$ such that $K^{j} \cap C \subseteq \Pi_{n_{j}}\left(U_{1}\right) \cup \cdots \cup \Pi_{n_{j}}\left(U_{q}\right)$ and for each $p$, the set $U_{p}$ is definable in $\mathcal{P}(\mathcal{R})$ and $\operatorname{dim} \Pi_{n_{j}}\left(U_{p}\right) \leq \operatorname{dim} K$.

Assuming Claim 1 holds, the proposition follows by applying Claim 1 to each $C \in C_{M}^{0}$. To prove Claim 1, we proceed by reverse induction on $\operatorname{dim} C \leq m$. Let

$$
\mathcal{D}_{C}:=\left\{D^{\prime} \cap\left(\pi_{j}^{j+1}\right)^{-1}(C): D^{\prime} \in \mathcal{C}_{M}^{j+1}, C \subseteq \pi_{j}^{j+1}\left(D^{\prime}\right)\right\},
$$


and fix an arbitrary $D \in \mathcal{D}_{C}$; it suffices to prove Claim 1 with $K^{j+1}$ and $D$ in place of $K^{j}$ and $C$. Let $D^{\prime} \in \mathcal{C}_{M}^{j+1}$ be such that $D \subseteq D^{\prime}$; if $\operatorname{dim} D^{\prime}>\operatorname{dim} C$, then Claim 1 with $K^{j+1}$ and $D$ in place of $K^{j}$ and $C$ follows from the inductive hypothesis, so we also assume that $\operatorname{dim} D^{\prime}=\operatorname{dim} C$. Then $D$ is open in $D^{\prime}$, and since $M^{j+1} \subseteq \mathbb{R}^{n_{j}} \times G_{n_{j}}^{m-k}$ and $G_{n_{j}}^{m-k}$ is compact, there is a definable map $g: C \longrightarrow G_{n_{j}}^{m-k}$ such that $D=\operatorname{gr} g$. Let

$$
W:=\bigcup\left\{E \in \mathcal{C}_{M}^{j+1}: \operatorname{dim} E>\operatorname{dim} C\right\} ;
$$

since $\mathcal{C}^{j+1}$ is a stratification, both $W$ and $W \cup D^{\prime}$ are open in $\mathrm{cl} M^{j+1}$, and since $D$ is open in $D^{\prime}$, the set $W \cup D$ is also open in cl $M^{j+1}$. Hence by Proposition 5.3, the set $\left(K^{j+1} \cap D\right) \backslash\left(\lim _{\iota} F^{j+1} V_{\iota} \cup \operatorname{fr}\left(W \cap K^{j+1}\right)\right)$ is an embedded integral manifold of $g^{1}$, where $g_{1}: D \longrightarrow G_{n_{j+1}}^{m-k}$ is the pullback of $g$ via the restriction of $\pi_{j}^{j+1}$ to $D$. But $D \cap \operatorname{fr}\left(W \cap K^{j+1}\right) \subseteq F$, where

$$
F:=\bigcup\left\{\operatorname{fr}\left(E \cap K^{j+1}\right): E \in \mathcal{C}_{M}^{j+1} \text { and } \operatorname{dim} E>\operatorname{dim} C\right\}
$$

is compact, so the set

$$
L:=\left(K^{j+1} \cap D\right) \backslash\left(\lim _{\iota} F^{j+1}\left(V_{\iota}\right) \cup F\right)
$$

is a finite union of connected integral manifolds of $g^{1}$ definable in $\mathcal{P}(\mathcal{R})$. Thus by (I) and (II), to prove Claim 1 with $K^{j+1}$ and $D$ in place of $K^{j}$ and $C$, it now suffices to prove:

Claim 2: There is a $q \in \mathbb{N}$ and, for $p=1, \ldots, q$, there are $m_{p} \geq n_{j+1}$ and an integral manifold $U_{p} \subseteq \mathbb{R}^{m_{p}}$ over $\mathcal{R}$ such that $L \subseteq \Pi_{n_{j+1}}\left(U_{1}\right) \cup \cdots \cup \Pi_{n_{j+1}}\left(U_{q}\right)$ and for each $p$, the set $U_{p}$ is definable in $\mathcal{P}(\mathcal{R})$ and $\operatorname{dim} \Pi_{n_{j+1}}\left(U_{p}\right) \leq \operatorname{dim} L$.

For the proof of Claim 2, we write $n, d$ and $V_{\iota}$ in place of $n_{j+1}, d^{j+1}$ and $V_{\iota}^{j+1}$. Since $\sim_{d_{k}}$ is not definable, the nested distribution $d^{\prime}:=\left(d_{0}, \ldots, d_{k-1}\right)$ satisfies $\operatorname{deg} d^{\prime}<\operatorname{deg} d$. Moreover, there is an admissible sequence $\left(U_{\iota}\right)$ of integral manifolds of $d^{\prime}$ such that $V_{\iota} \subseteq U_{\iota}$ for all $\iota$. Passing to a subsequence if necessary, we may assume that $Y:=\lim _{\iota} U_{\iota}$ exists; in particular, note that $L \subseteq Y$. By the inductive hypothesis, there is a $q \in \mathbb{N}$ and, for $p=1, \ldots, q$, there exist $m_{p} \geq n$, a definable $C^{2}$-manifold $N_{p} \subseteq \mathbb{R}^{m_{p}}$, a definable nested distribution $f_{p}=\left(f_{p, 0}, \ldots, f_{p, k(p)}\right)$ on $N_{p}$ and a nested integral manifold $Z_{p}=$ $\left(Z_{p, 0}, \ldots, Z_{p, k(p)}\right)$ of $f_{p}$ definable in $\mathcal{P}(\mathcal{R})$ such that

$$
Y \subseteq \Pi_{n}\left(Z_{1, k(1)}\right) \cup \cdots \cup \Pi_{n}\left(Z_{q, k(q)}\right)
$$

and $\operatorname{dim} \Pi_{n}\left(Z_{p, k(p)}\right) \leq \operatorname{dim} Y$ for each $p$. After refining and pruning the collection $\left\{N_{1}, \ldots, N_{q}\right\}$ if necessary, we may assume that that

$(\dagger) \Pi_{n}\left(N_{p}\right) \subseteq D$ and $\Pi_{n}\left(Z_{p, k(p)}\right) \cap L \neq \emptyset$ for each $p$, and that $L \subseteq$ $\Pi_{n}\left(Z_{1, k(1)}\right) \cup \cdots \cup \Pi_{n}\left(Z_{q, k(q)}\right)$. 
We now prove Claim 2 by induction on

$$
\delta:=\max \left\{\operatorname{dim} \Pi_{n}\left(Z_{p, k(p)}\right): p=1, \ldots, q\right\},
$$

simultaneously for all such collections $\left\{\left(N_{p}, f_{p}, Z_{p}\right)\right\}$ satisfying $(\dagger)$. If $\delta=$ $\operatorname{dim} L$, we take $U_{p}:=Z_{p, k(p)}$ for each $p$ and are done. So assume $\delta>\operatorname{dim} L$ and Claim 2 holds for lower values of $\delta$. Refining and pruning the collection $\left\{N_{1}, \ldots, N_{q}\right\}$ again if necessary, we may assume for each $p$, each $\mu \leq n$ and each strictly increasing $\lambda:\{1, \ldots, \mu\} \longrightarrow\{1, \ldots, n\}$ that the dimension of the spaces

$$
\Pi_{\lambda}\left(f_{p, k(p)}(y)\right) \text { and } \Pi_{\lambda}\left(\Pi_{n}\left(f_{p, k(p)}(y)\right) \cap g^{1}\left(\Pi_{n}(y)\right)\right)
$$

is constant as $y$ ranges over $N_{p}$. Then by Proposition 6.3 with $\nu=n$, for each $p$ such that $\operatorname{dim} \Pi_{n}\left(Z_{p, k(p)}\right)=\delta$, the integral manifold $Z_{p, k(p)}$ over $\mathcal{R}$ can be replaced by finitely many integral manifolds $Z^{\prime}$ over $\mathcal{R}$ contained in $N_{p}$ such that $\operatorname{dim} \Pi_{n}\left(Z^{\prime}\right)<\delta$. Claim 2 then follows from the inductive hypothesis, and the proposition is proved.

\section{Normal SETS AND REgular Closure}

We assume from now on that $\mathcal{R}$ admits analytic cell decomposition. In this section, we denote by $\|\cdot\|$ the Euclidean norm on $\mathbb{R}^{k}$ (for $k=1,2, \ldots$ ).

Definition 8.1. A definable open set $U \subseteq \mathbb{R}^{n}$ is normal if there exists an analytic, definable carpeting function on $U$.

Remark. If $U, V \subseteq \mathbb{R}^{n}$ are normal, then so are $U \cap V$ and $U \times V$.

Example 8.2. Every analytic, open definable cell is normal.

Proposition 8.3. Let $A \subseteq \mathbb{R}^{n}$ be open and definable. Then $A$ is a union of finitely many normal sets.

Proof. By induction on $n$; we may assume that $A \neq \mathbb{R}^{n}$. The case $n=0$ is trivial, so we assume that $n>0$ and that the proposition holds for lower values of $n$. By analytic cell decomposition, it suffices to show that every definable analytic cell contained in $A$ is contained in a finite union of normal sets contained in $A$.

So we let $C \subseteq A$ be a definable analytic cell; we proceed by induction on the dimension $c$ of $C$. If $c=0$, then $C$ is a singleton and any ball centered at $C$ and contained in $A$ will do. So we assume that $c>0$ and that every analytic cell of dimension less than $c$ contained in $A$ is contained in a finite union of normal sets contained in $A$. If $c=n$, then $C$ is open and hence normal by Example 8.2; so we also assume that $c<n$.

After permuting coordinates if necessary, there is an open, analytic cell $D \subseteq \mathbb{R}^{c}$ and a definable, analytic map $g: D \longrightarrow \mathbb{R}^{n-c}$ such that $C=$ gr $g$. For $x \in \mathbb{R}^{n}$, we write $x=(y, z)$ with $y \in \mathbb{R}^{c}$ and $z \in \mathbb{R}^{n-c}$. Define $\beta: D \longrightarrow(0, \infty)$ 
by $\beta(y):=\operatorname{dist}\left((y, g(y)), \mathbb{R}^{n} \backslash A\right)$, where this distance is computed using the Euclidean norm. By the inductive hypothesis and analytic cell decomposition, we may assume that $\beta$ is analytic. Now we put

$$
U:=\left\{(y, z) \in A: y \in D \text { and }\|z-g(y)\|^{2}<\beta^{2}(y)\right\} .
$$

This $U$ is normal: given an analytic, definable carpeting function $\gamma: D \longrightarrow$ $(0, \infty)$, we define $\phi: U \longrightarrow(0, \infty)$ by

$$
\phi(y, z):=\gamma(y)\left(\beta^{2}(y)-\|z-g(y)\|^{2}\right),
$$

which is easily seen to be an analytic, definable carpeting function on $U$.

Definition 8.4. Let $U \subseteq \mathbb{R}^{n}$ be normal and $A \subseteq U$.

(1) $A$ is normal in $U$ if $A$ is a finite union of sets of the form

$$
\{x \in U: g(x)=0, h(x)>0\},
$$

where $g: U \longrightarrow \mathbb{R}^{q}$ and $h: U \longrightarrow \mathbb{R}^{r}$ are definable and analytic and " $h(x)>0 "$ means " $h_{s}(x)>0$ for $s=1, \ldots, r$ ".

(2) $A$ is a normal leaflet in $U$ (of codimension $p$ ) if

$$
A=\{x \in U: f(x)=g(x)=0, h(x)>0\},
$$

where $f: U \longrightarrow \mathbb{R}^{p}, g: U \longrightarrow \mathbb{R}^{q}$ and $h: U \longrightarrow \mathbb{R}^{r}$ are definable and analytic and for all $x \in A$, the rank of $f$ at $x$ is $p$ and $\operatorname{ker} d f(x) \subseteq$ ker $d g(x)$.

Remark 8.5. In the situation of Definition 8.4(2), the set $A$ is an analytic submanifold of $U$ of dimension $n-p$; in fact, we have $T_{x} A=\operatorname{ker} d f(x)$ for all $x \in A$. Moreover, if $\delta: U \longrightarrow(0, \infty)$ is an analytic, definable carpeting function on $U$, then the restriction of $\phi:=\delta \cdot \prod_{s=1}^{r} H_{s}: U \longrightarrow \mathbb{R}$ to $A$ is an analytic carpeting function on $A$, where $H_{s}:=h_{s} / \sqrt{1+h_{s}^{2}}$ for each $s$.

Example 8.6. Let $U \subseteq \mathbb{R}^{n}$ be normal; then $U$ is a normal leaflet in $U$. Let also $f: U \longrightarrow \mathbb{R}^{p}$ and $h: U \longrightarrow \mathbb{R}^{r}$ be analytic and definable. Then the set

$$
\begin{aligned}
& \{x \in U: f(x)=0, h(x)>0 \text { and } f \text { has rank } p \text { at } x\} \\
& =\left\{x \in U: f(x)=0, h(x)>0,|d f|^{2}(x)>0\right\} \\
& =\left\{x \in U: f(x)=0, h(x)>0, d f_{1}(x) \wedge \cdots \wedge d f_{p}(x) \neq 0\right\}
\end{aligned}
$$

is a normal leaflet in $U$, where $|d f|^{2}$ denotes the sum of the squares of all $(p \times p)$-subdeterminants of $d f$.

The following lemma is elementary, and its proof is left to the reader.

Lemma 8.7. Let $U \subseteq \mathbb{R}^{n}$ and $V \subseteq \mathbb{R}^{m}$ be normal.

(1) If $A$ and $B$ are normal in $U$, then so are $A \cup B, A \cap B$ and $A \backslash B$. 
(2) Let $A$ be normal in $U$ and $B$ be normal in $V$. Then $A \times B$ is normal in $U \times V$. Moreover, if $A$ and $B$ are normal leaflets in $U$ and $V$, respectively, then $A \times B$ is a normal leaflet in $U \times V$.

(3) Let $\phi: U \longrightarrow V$ be definable and analytic, and let $B$ be normal in $V$. Then $\phi^{-1}(B)$ is normal in $U$.

(4) Let $A$ be a normal leaflet in $U$ of codimension $p$, and assume that there is a definable, analytic embedding $\phi: A \longrightarrow \mathbb{R}^{n-p}$. Then $\phi(A)$ is normal. Moreover, if $B \subseteq A$ is normal (resp., a normal leaflet) in $U$, then $\phi(B)$ is normal (resp., a normal leaflet) in $\phi(A)$.

The following lemma will be used several times in this section:

Lemma 8.8. Let $\eta: X \longrightarrow(0, \infty)$ be definable in $\mathcal{P}(\mathcal{R})$ and put

$$
Y:=\{(x, t): x \in X \text { and } 0<t<\eta(x)\} .
$$

Let also $\alpha: Y \longrightarrow[0, \infty)$ be definable in $\mathcal{P}(\mathcal{R})$, and assume that for every $x \in X$, the function $\alpha_{x}:(0, \eta(x)) \longrightarrow[0, \infty)$ defined by $\alpha_{x}(t):=\alpha(x, t)$ is semianalytic. Then there exists an $N \in \mathbb{N}$ such that for all $x \in X$, either ultimately $\alpha_{x}(t)=0$ or ultimately $\alpha_{x}(t)>t^{N}$ (where "ultimately" abbreviates "for all sufficiently small $t>0 ")$.

Proof. By monotonicity, for every $x \in X$ the function $\alpha_{x}$ is ultimately of constant sign. By Puiseux's Theorem, for every $x \in X$ such that $\alpha_{x}$ is ultimately positive, there are $c_{x}>0$ and $r_{x} \in \mathbb{Q}$ such that ultimately $\alpha_{x}(t)=c_{x} t^{r_{x}}+o\left(t^{r_{x}}\right)$. However, the set $R_{X}:=\left\{r_{x} \in \mathbb{R}: x \in X\right\}$ is definable, since for all $x \in X$ such that $\alpha_{x}$ is ultimately positive, we have $r_{x}=\lim _{t \rightarrow 0^{+}} t \alpha_{x}^{\prime}(t) / \alpha_{x}(t)$. Since each $r_{x}$ is rational, it follows that $R_{X}$ is finite, so any $N>\max R_{X}$ will do.

Proposition 8.9 (Gabrielov [9]). Let $U \subseteq \mathbb{R}^{n}$ be normal and $A$ be normal in $U$. Then $A$ is a finite union of normal leaflets in $U$.

Proof. Let $g: U \longrightarrow \mathbb{R}^{q}$ and $h: U \longrightarrow \mathbb{R}^{r}$ be definable and analytic such that $A=\{x \in U: g(x)=0, h(x)>0\}$; we proceed by induction on $d:=\operatorname{dim} A$. If $d=0$, the proposition is trivial, so we assume that $d>0$ and the proposition holds for lower values of $d$.

Let $\mathcal{C}$ be a finite stratification of $A$ into analytic cells, and put $\mathcal{C}_{d}:=$ $\{C \in \mathcal{C}: \operatorname{dim} C=d\}$. We show that for $C \in \mathcal{C}_{d}$, there is a normal leaflet $A_{C} \subseteq A$ such that $\operatorname{dim}\left(C \backslash A_{C}\right)<d$. The proposition then follows from the inductive hypothesis, since $A \backslash \bigcup_{C \in \mathcal{C}_{d}} A_{C}$ is a normal subset of $U$ of dimension less than $d$.

Fix $C \in \mathcal{C}_{d}$, and let $\mathcal{G}$ be the set of all partial derivatives (of all orders) of $g_{1}, \ldots, g_{q}$. Let $M$ be the set of all natural numbers $m$ for which there exist $f_{1}, \ldots, f_{m} \in \mathcal{G}$ such that $C \subseteq\left\{x \in U: f_{1}(x)=\cdots=f_{m}(x)=0\right\}$ and $d f_{1}(a) \wedge \cdots \wedge d f_{m}(a) \neq 0$ for some $a \in C$. 
Put $p:=\sup M \leq n-d$; we claim that $p=n-d$. To see this, let $f_{1}, \ldots, f_{p} \in \mathcal{G}, a \in C$ and an open ball $B$ centered at $a$ be such that $C \cap B$ is a connected submanifold of

$$
\Gamma:=\left\{x \in U: f_{1}(x)=\cdots=f_{p}(x)=0\right\} \cap B,
$$

and such that $\Gamma$ is a connected, analytic submanifold of codimension $p$ contained in $\left\{x \in U: h(x)>0, d f_{1}(x) \wedge \cdots \wedge d f_{p}(x) \neq 0\right\}$. The maximality of $p$ now implies that $g(x)=0$ for all $x \in \Gamma$. Since $\mathcal{C}$ is a stratification of $A$ and $\operatorname{dim} C=d$, the cell $C$ is an open subset of $A$; so after shrinking $B$ if necessary, we may assume that $C \cap B=A \cap B$. It follows that $A \cap B \subseteq \Gamma$, that is, $C \cap B=A \cap B=\Gamma$, which proves the claim.

Put $f:=0$ if $p=0$ and $f:=\left(f_{1}, \ldots, f_{p}\right)$ otherwise, where $f_{1}, \ldots, f_{p} \in \mathcal{G}$ are as in the previous paragraph. Let

$$
V:=\left\{x \in U: f(x)=0, h(x)>0,|d f|^{2}(x)>0\right\},
$$

a normal leaflet in $U$. Note that $C \backslash V$ is contained in the set $\{x \in U: f(x)=$ $\left.0,|d f|^{2}(x)=0\right\}$, and the latter has dimension strictly less than $d$. The only remaining problem, therefore, is that $V$ is not necessarily a subset of $A$. To address this issue, we let $\eta: V \longrightarrow(0, \infty)$ be a definable function such that $B(x, 2 \eta(x)) \subseteq\left\{x \in U: h(x)>0,|d f|^{2}(x)>0\right\}$ for all $x \in V$. For $x \in V$ and $t \in(0, \eta(x))$, we put

$$
\alpha(x, t):=\max \{|g|(y): y \in V,\|y-x\| \leq t\} .
$$

Note that $\alpha$ is definable, and for $x \in V$ the function $\alpha_{x}:(0, \eta(x)) \longrightarrow[0, \infty)$ is semianalytic. Hence by Lemma 8.8, there is an $N \in \mathbb{N}$ such that for all $x \in V$, either ultimately $\alpha_{x}(t)=0$ or ultimately $\alpha_{x}(t)>t^{N}$.

We now let $A_{C}$ be the subset of $V$ where all partials of $g \uparrow_{V}$ up to order $N$ vanish. More precisely, we let $\mathcal{S}$ be the set of all $\phi: U \longrightarrow \mathbb{R}$ for which there exist $\nu \leq N$ and functions $\phi_{0}, \ldots, \phi_{\nu}: U \longrightarrow \mathbb{R}$ such that $\phi=\phi_{\nu}$, $\phi_{0} \in\left\{g_{1}, \ldots, g_{q}\right\}$ and for $i \in\{1, \ldots, \nu\}$, the function $\phi_{i}$ is one of the coefficient functions of $d \phi_{i-1} \wedge d f_{1} \wedge \cdots \wedge d f_{p}$. Then we put

$$
A_{C}:=\{x \in V: \phi(x)=0 \text { for all } \phi \in \mathcal{S}\} .
$$

It follows for all $x \in V$ that $x \in A_{C}$ if and only if ultimately $\alpha_{x}(t) \leq t^{N}$, that is, if and only if ultimately $\alpha_{x}(t)=0$, that is, if and only if $g=0$ in a neighbourhood of $x$ in $V$. Hence $A_{C} \subseteq A$, and the only points $x \in V \cap A$ that are not contained in $A_{C}$ are those where $g$ is not identically 0 on any neighbourhood of $x$ in $V$. Thus, $\operatorname{dim}\left(C \backslash A_{C}\right)<d$, and since $A_{C}$ is a leaflet in $U$, the proposition is proved.

Corollary 8.10. Let $U \subseteq \mathbb{R}^{n}$ be normal and $\mathcal{D}$ be a finite collection of definable, analytic distributions on $U$, and let $A$ be a normal subset of $U$. Then $A$ is a finite union of normal leaflets in $U$ that are compatible with $\mathcal{D}$. 
Proof. By induction on $\operatorname{dim} A$; if $\operatorname{dim} A=0$, there is nothing to do, so we assume $\operatorname{dim} A>0$ and the corollary is true for lower values of $\operatorname{dim} A$. By Proposition 8.9 and the inductive hypothesis, we may assume that $A$ is a normal leaflet in $U$. Proceeding as in the proof of Proposition 2.2, we note that for $\mathcal{E} \subseteq \mathcal{D}$ and $i \in\{0, \ldots, \operatorname{dim} A\}$, the set $A_{\mathcal{E}, i}:=\left\{x \in A: \operatorname{dim} T_{x} \mathcal{E}=i\right\}$ is a normal subset of $U$. Again by Proposition 8.9, there is a finite collection $\mathcal{C}$ of normal leaflets in $U$ such that each $A_{\mathcal{E}, i}$ is a union of leaflets in $\mathcal{C}$. Arguing as in the proof of Proposition 2.2. we see that every leaflet in $\mathcal{C}$ of dimension $\operatorname{dim} A$ is compatible with $\mathcal{D}$. The corollary now follows from the inductive hypothesis.

We next obtain a fiber cutting lemma for nested Rolle leaves (Corollary 8.14 below), using normal leaflets and building on the techniques found in Moussu and Roche [18, Lion and Rolin [13] and [20]. We let $U \subseteq \mathbb{R}^{n}$ be a normal set and $A$ a normal subset of $U$. We also let $\Delta=\left\{d^{1}, \ldots, d^{q}\right\}$ be a set of definable, analytic nested distributions on $U$; we write $d^{p}=\left(d_{0}^{p}, \ldots, d_{k(p)}^{p}\right)$ for $p=1, \ldots, q$ and associate $\mathcal{D}_{\Delta}$ to $\Delta$ as in Section 3 .

Lemma 8.11. Assume that $A$ is a normal leaflet in $U$ compatible with $\mathcal{D}_{\Delta}$, and suppose that $\operatorname{dim} d_{k(\Delta, A)}^{\Delta, A}>0$. Then there is an analytic, definable $\phi$ : $U \longrightarrow \mathbb{R}$ such that $\phi \uparrow_{A}$ is a carpeting function on $A$ and the definable set

$$
B:=\left\{x \in A: d_{k(\Delta, A)}^{\Delta, A}(x) \subseteq \operatorname{ker} d\left(\phi \uparrow_{A}\right)(x)\right\}
$$

has dimension less than $\operatorname{dim} A$.

Proof. Let $\psi: U \longrightarrow \mathbb{R}$ be analytic and definable such that $\psi \uparrow_{A}$ is a carpeting function on $A$ (as obtained in Remark 8.5, say). For $u \in(0, \infty)^{n}$, we define $\psi_{u}: U \longrightarrow \mathbb{R}$ by $\psi_{u}(x):=\psi(x) \phi_{u}(x)$, where $\phi_{u}(x)$ is as in Section 1; note that $\psi_{u} \uparrow_{A}$ is an analytic, definable carpeting function on $A$. Now consider the definable set

$$
D:=\left\{(u, x) \in \mathbb{R}^{n} \times A: d_{k(\Delta, A)}^{\Delta, A}(x) \subseteq \operatorname{ker} d\left(\psi_{u}\left\lceil_{A}\right)(x)\right\} .\right.
$$

Arguing as in the proof of Lemma 3.1, we conclude that $\operatorname{dim} D_{u}<\operatorname{dim} A$ for some $u \in(0, \infty)^{n}$, so we take $\phi:=\psi_{u}$.

For $I \subseteq\{1, \ldots, q\}$ we put $\Delta(I):=\left\{d^{p}: p \in I\right\}$.

Lemma 8.12. Let $I \subseteq\{1, \ldots, q\}$. Then there is a finite collection $\mathcal{P}$ of normal leaflets in $U$ contained in $A$ such that $\mathcal{P}$ is compatible with $\mathcal{D}_{\Delta(J)}$ for every $J \subseteq\{1, \ldots, q\}$ and

(i) $\operatorname{dim} d_{k(\Delta(I), N)}^{\Delta(I), N}=0$ for every $N \in \mathcal{P}$;

(ii) whenever $V_{p}$ is a Rolle leaf of $d^{p}$ for $p \in I$, every component of $A \cap$ $\bigcap_{p \in I} V_{p}$ intersects some leaflet in $\mathcal{P}$. 
Proof. By induction on $\operatorname{dim} A$; if $\operatorname{dim} A=0$, there is nothing to do, so we assume $\operatorname{dim} A>0$ and the corollary is true for lower values of $\operatorname{dim} A$. By Corollary 8.10 and the inductive hypothesis, we may assume that $A$ is a normal leaflet in $U$ compatible with $\mathcal{D}_{\Delta(J)}$ for $J \subseteq\{1, \ldots, q\}$. Thus, if $\operatorname{dim} d_{k(\Delta(I), A)}^{\Delta(I), A}=$ 0 , we are done; otherwise, we let $\phi$ and $B$ be as in Lemma 8.11 with $\Delta(I)$ in place of $\Delta$.

Let $V_{p}$ be a Rolle leaf of $d^{p}$ for each $p$. By Proposition 8.9 and the inductive hypothesis, it now suffices to show that every component of $X:=A \cap \bigcap_{p \in I} V_{p}$ intersects $B$. However, since $d_{k(\Delta(I), A)}^{\Delta(I), A}$ has dimension, $X$ is a closed, embedded submanifold of $A$. Thus, $\phi$ attains a maximum on every component of $X$, and any point in $X$ where $\phi$ attains a local maximum belongs to $B$.

Corollary 8.13. Let $d$ be a definable, analytic nested distribution on $U$ and $m \leq n$. Then there is a finite collection $\mathcal{P}$ of normal leaflets in $U$ contained in $A$ such that for every Rolle leaf $V$ of $d$, we have

$$
\Pi_{m}(A \cap V)=\bigcup_{N \in \mathcal{P}} \Pi_{m}(N \cap V)
$$

and for every $N \in \mathcal{P}$, the set $N \cap V$ is an analytic submanifold of $U$, $\Pi_{m} \uparrow_{(N \cap V)}$ is an immersion and for every $n^{\prime} \leq n$ and every strictly increasing $\lambda:\left\{1, \ldots, n^{\prime}\right\} \longrightarrow\{1, \ldots, n\}$, the projection $\left.\Pi_{\lambda}\right|_{(N \cap V)}$ has constant rank.

Proof. Apply Lemma 8.12 with $q:=n+1, d^{p}:=\operatorname{ker} d x_{p}$ for $p=1, \ldots, n$, $d^{q}:=d$ and $I:=\{1, \ldots, m, n+1\}$.

Corollary 8.14. Let $M \subseteq \mathbb{R}^{n}$ be a definable $C^{2}$-manifold, $d$ be a definable nested distribution on $M, B \subseteq \mathbb{R}^{n}$ be a definable set and $m \leq n$. Then there is a finite collection $\mathcal{P}$ of definable analytic manifolds contained in $B$ such that for every Rolle leaf $V$ of $d$, we have

$$
\Pi_{m}(B \cap V)=\bigcup_{N \in \mathcal{P}} \Pi_{m}(N \cap V)
$$

and for $N \in \mathcal{P}$, the set $N \cap V$ is an analytic manifold and a submanifold of $M$, $\Pi_{m} \Upsilon_{(N \cap V)}$ is an immersion and for every $n^{\prime} \leq n$ and every strictly increasing $\lambda:\left\{1, \ldots, n^{\prime}\right\} \longrightarrow\{1, \ldots, n\}$, the projection $\Pi_{\lambda} \uparrow_{(N \cap V)}$ has constant rank.

Proof. By analytic cell decomposition and Corollary 3.3.1), we may assume that $M=B$ is an analytic cell and $d$ is analytic. Let $\mu:\{1, \ldots, \operatorname{dim} M\} \longrightarrow$ $\{1, \ldots, n\}$ be such that $\Pi_{\mu}(M)$ is open and $\Pi_{\mu} \uparrow_{M}: M \longrightarrow \Pi_{\mu}(M)$ is an analytic diffeomorphism. Then $\Pi_{\mu}(M)$ is normal and $M$ is the graph of an analytic function $g: \Pi_{\mu}(M) \longrightarrow \mathbb{R}^{n-\operatorname{dim} M}$. The corollary now follows from Corollary 8.13 applied to the push-forward of $d$ via $\Pi_{\mu} \uparrow_{M}$. 
Regular closure. Let $U \subseteq \mathbb{R}^{n}$ be a normal set and $A$ a normal subset of $U$. Let also $d=\left(d_{0}, \ldots, d_{k}\right)$ be a definable, integrable, analytic nested distribution on $U$ and $V$ be a Rolle leaf of $d$. Following [9], we study below the closure in $U$ of $A \cap V$, defined by $\operatorname{cl}_{U}(A \cap V):=U \cap \operatorname{cl}(A \cap V)$, and the frontier in $U$ of $A \cap V$, defined by $\operatorname{fr}_{U}(A \cap V):=U \cap \operatorname{fr}(A \cap V)$.

Proposition 8.15. There are normal subsets $B$ and $C$ of $U$ such that

$$
\operatorname{cl}_{U}(A \cap V)=B \cap V \text { and } \operatorname{fr}_{U}(A \cap V)=C \cap V .
$$

For the proof of Proposition 8.15, we need the following preliminary observations; here we consider $\Sigma_{n}$ as a definable subset of $\mathbb{R}^{2 n}$. For every $\sigma \in \Sigma_{n}$, we let $U_{\sigma}$ be the set of all $x \in U$ such that $\sigma\left(d_{k}(x)\right)$ is the graph of a linear map $L: \mathbb{R}^{n-k} \longrightarrow \mathbb{R}^{k}$ satisfying $|L|<2$. Then $U=\bigcup_{\sigma \in \Sigma_{n}} U_{\sigma}$ by Lemma 1.3, and by definable choice there is a definable map $x \mapsto \sigma_{x}: U \longrightarrow \Sigma_{n}$ such that $x \in U_{\sigma_{x}}$ for all $x \in U$. Since each $U_{\sigma}$ is open, there is a definable map $x \mapsto \epsilon(x): U \longrightarrow(0, \infty)$ such that $y \in U_{\sigma_{x}}$ for all $y \in B(x, 4(n-k) \epsilon(x))$. We put

$$
G:=\{(x, y) \in U \times U: y \in B(x, 2(n-k) \epsilon(x))\}
$$

and define $L: G \longrightarrow G L\left(\mathbb{R}^{n-k}, \mathbb{R}^{k}\right)$ such that $\sigma_{x}\left(d_{k}(y)\right)$ is the graph of $L(x, y)$ for all $(x, y) \in G$. Then $L$ is definable and for every $x \in U$, the map $L_{x}$ : $B(x, 2(n-k) \epsilon(x)) \longrightarrow G L\left(\mathbb{R}^{n-k}, \mathbb{R}^{k}\right)$ defined by $L_{x}(y):=L(x, y)$ is analytic.

Below, we set $x_{-}:=x_{\leq n-k}$ and $x_{+}:=x_{>n-k}$ and

$$
B^{\sigma}(x, \epsilon):=\sigma_{x}^{-1}\left(B\left(x_{-}, \epsilon\right) \times B\left(x_{+}, 2(n-k) \epsilon\right)\right)
$$

for $x \in U$ and $\epsilon>0$. Also for $x \in U$, we denote by $V_{x}$ the leaf of $d_{k}^{B^{\sigma}(x, \epsilon(x))}$ containing $x$. By Lemma 1.4 and because $V_{x}$ is an analytic manifold, the set $\sigma_{x}\left(V_{x}\right)$ is the graph of an analytic, $2(n-k)$-Lipschitz map $F_{x}: B\left(x_{-}, \epsilon(x)\right) \longrightarrow$ $B\left(x_{+}, 2(n-k) \epsilon(x)\right)$ such that

$$
d F_{x}(z)=L_{x}\left(z, F_{x}(z)\right) \quad \text { for all } z \in B\left(x_{-}, \epsilon(x)\right) .
$$

For the next lemma, we put

$$
G_{0}:=\left\{(x, y) \in U \times \mathbb{R}^{n}:|y|<2(n-k) \epsilon(x)\right\} .
$$

Lemma 8.16. For $\nu \in \mathbb{N}$ there is a definable map $P_{\nu}: G_{0} \longrightarrow \mathbb{R}^{k}$ such that for every $x \in U$,

(i) the map $P_{\nu, x}: B(0,2(n-k) \epsilon(x)) \longrightarrow \mathbb{R}^{k}$ defined by $P_{\nu, x}(y):=P_{\nu}(x, y)$ is a homogeneous polynomial of degree $\nu$ in $y$;

(ii) $\sum_{\nu=0}^{\infty} P_{\nu, x}(y-x)$ converges to an analytic map $\phi_{x}: B^{\sigma}(x, \epsilon(x)) \longrightarrow \mathbb{R}^{k}$ definable in $\mathcal{P}(\mathcal{R})$ such that $\sigma_{x}\left(V_{x}\right)=\left\{y \in B^{\sigma}\left(x, \eta_{x}\right): \phi_{x}(y)=0\right\}$.

Proof. Differentiating (8.1) with respect to $z$, one finds by induction on $|\alpha|=$ $\alpha_{1}+\cdots+\alpha_{n-k}$ for $\alpha \in \mathbb{N}^{n-k}$ that there is a definable function $L^{\alpha}: G \longrightarrow \mathbb{R}$ 
such that for all $x \in U$ and $z \in B\left(x_{-}, \epsilon(x)\right)$,

$$
L^{\alpha}\left(x, z, F_{x}(z)\right)=\frac{1}{\alpha !} \frac{\partial^{\alpha}}{\partial z^{\alpha}} F_{x}(z)
$$

and such that for all $x \in U$, the function $L_{x}^{\alpha}: B\left(x, 2 \eta_{x}\right) \longrightarrow \mathbb{R}$ defined by $L_{x}^{\alpha}(y):=L^{\alpha}(x, y)$ is analytic. For $x \in U$, we now define $\phi_{x}: B^{\sigma}\left(x, \eta_{x}\right) \longrightarrow \mathbb{R}^{k}$ by $\phi_{x}(y):=\sigma_{x}(y)_{+}-F_{x}\left(\sigma_{x}(y)_{-}\right)$; then $\phi_{x}$ is analytic and definable in $\mathcal{P}(\mathcal{R})$, and $\sigma_{x}\left(V_{x}\right)=\left\{y \in B^{\sigma}\left(x, \eta_{x}\right): \phi_{x}(y)=0\right\}$. Moreover, from Taylor expansion we get $\phi_{x}(y)=\sum_{q=0}^{\infty} P_{q, x}(y-x)$, where $P_{0, x}(y):=\sigma_{x}(x)_{+}-F_{x}\left(\sigma_{x}(x)_{-}\right)$, $P_{1, x}(y):=\sigma_{x}(y)_{+}-L_{x}\left(\sigma_{x}(x)_{-}\right) \cdot \sigma_{x}(y)_{-}$and

$$
P_{\nu, x}(y):=\sum_{|\alpha|=\nu} L_{x}^{\alpha}\left(\sigma_{x}(x)_{-}, F_{x}\left(\sigma_{x}(x)_{-}\right)\right) \cdot\left(y_{-}\right)^{\alpha} \quad \text { for } \nu>1
$$

hence $P_{\nu}(x, y):=P_{\nu, x}(y)$ will do.

Given an analytic map $h=\left(h_{1}, \ldots, h_{l}\right): U \longrightarrow \mathbb{R}^{l}, \nu \in \mathbb{N}$ and $x \in U$, we denote by $h_{x}^{\nu}: U-x \longrightarrow \mathbb{R}^{l}$ the Taylor expansion of order $\nu$ of $h$ at $x$ and by $h_{\min }: U \longrightarrow \mathbb{R}$ the function $h_{\min }(x):=\min \left\{h_{1}(x), \ldots, h_{l}(x)\right\}$.

Proof of Proposition 8.15. Assume that

$$
A=\{x \in U: g(x)=0, h(x)>0\},
$$

with $g: U \longrightarrow \mathbb{R}^{q}$ and $h: U \longrightarrow \mathbb{R}^{r}$ definable and analytic, and put $Z(h):=$ $\{x \in U: h(x)=0\}$. It suffices to find a normal set $C \subseteq Z(h)$ in $U$ such that $\operatorname{fr}_{U}(A \cap V)=C \cap V$, since then $B:=A \cup C$ will do. Below we work with the notations from Lemma 8.16 and the paragraphs preceding it.

Let $Y:=\{(x, t) \in U \times(0, \infty): x \in V \cap Z(h), 0<t<\epsilon(x)\}$. First, we define $\alpha: Y \longrightarrow[0, \infty)$ by

$$
\alpha(x, t):=\max \left(\left\{h_{\min }(y): y \in V, g(y)=0,\|y-x\| \leq t\right\} \cup\{0\}\right) .
$$

By Lemma 8.8, there exists an $N \in \mathbb{N}$ such that for all $x \in V \cap Z(h)$, either ultimately $\alpha_{x}(t)=0$ or ultimately $\alpha_{x}(t)>t^{N}$.

Fix an arbitrary $x \in V \cap Z(h)$. Then $x \in \operatorname{fr}(A \cap V)$ if and only if ultimately $\alpha_{x}(t)>t^{N}$. However, we have ultimately $\alpha_{x}(t)>t^{N}$ if and only if $x$ belongs to the closure of $\left\{y \in V: g(y)=0, h(y)>\|y-x\|^{N}>0\right\}$, and the latter holds if and only if $x$ belongs to the closure of $\left\{y \in D_{x}: g(y)=0\right\}$, where

$$
D_{x}:=\left\{y \in V: 2 h_{x}^{N}(y-x) \geq\|y-x\|^{N}>0\right\} .
$$

Second, we define $\beta: Y \longrightarrow[0, \infty)$ by

$$
\beta(x, t):=\min \left\{\left|\left(g(y), \phi_{x}(y)\right)\right|: 2 h_{x}^{N}(y-x) \geq\|y-x\|^{N},\|y-x\|=t\right\} .
$$

Again by Lemma 8.8, there exists an $M \in \mathbb{N}$ such that for all $x \in V \cap Z(h)$, either ultimately $\beta_{x}(t)=0$ or ultimately $\beta_{x}(t)>t^{M}$. 
Fix again an arbitrary $x \in V \cap Z(h)$. Then $x$ is not in the closure of $\left\{y \in D_{x}: g(y)=0\right\}$ if and only if ultimately $\beta_{x}(t)>t^{M}$. However, if ultimately $\beta_{x}(t)=0$, then $x$ is in the closure of

$$
\left\{y \in D_{x}: 4\left|\left(g(y), \phi_{x}(y)\right)\right|<\|y-x\|^{M}\right\}
$$

which implies that $x$ is in the closure of

$$
E_{x}:=\left\{y \in D_{x}: 2\left|\left(g_{x}^{M}(y-x),\left(\phi_{x}\right)_{x}^{M}(y-x)\right)\right|<\|y-x\|^{M}\right\} .
$$

Conversely, if $x \in \operatorname{cl} E_{x}$, then $x$ is in the closure of

$$
\left\{y \in D_{x}:\left|\left(g(y), \phi_{x}(y)\right)\right|<\|y-x\|^{M}\right\},
$$

which implies that ultimately $\beta_{x}=0$. It follows from the above that

(*) for all $x \in V \cap Z(h), x \in \operatorname{fr}(A \cap V)$ if and only if $x \in \operatorname{cl} E_{x}$.

Let $G \in \mathbb{R}[a, y]^{q}$ be the general $q$-tuple of polynomials in $y$ of degree $M$ and coefficients $a \in \mathbb{R}^{m_{1}}, H \in \mathbb{R}[b, y]^{r}$ be the general $r$-tuple of polynomials in $y$ of degree $N$ and coefficients $b \in \mathbb{R}^{m_{2}}$ and $\Phi \in \mathbb{R}[c, y]^{k}$ be the general $k$-tuple of polynomials in $y$ of degree $M$ and coefficients $c \in \mathbb{R}^{m_{3}}$. Let $S \subseteq$ $\mathbb{R}^{n} \times \mathbb{R}^{m_{1}} \times \mathbb{R}^{m_{2}} \times \mathbb{R}^{m_{3}} \times \mathbb{R}^{n}$ be the semialgebraic set

$$
\begin{aligned}
S:=\left\{(x, a, b, c, y): 2 H(b, y-x) \geq\|y-x\|^{N}>0\right. \\
\left.2|(G(a, y-x), \Phi(c, y-x))|<\|y-x\|^{M}\right\} .
\end{aligned}
$$

Then there are definable, analytic functions $a: U \longrightarrow \mathbb{R}^{m_{1}}, b: U \longrightarrow \mathbb{R}^{m_{2}}$ and $c: U \longrightarrow \mathbb{R}^{m-3}$ such that for all $x \in U$,

$$
E_{x}=\{y \in V:(x, a(x), b(x), c(x), y) \in S\} .
$$

Thus by (*), we have for $x \in V \cap Z(h)$ that $x \in \operatorname{fr}(A \cap V)$ if and only if $x \in \operatorname{cl} S_{(x, a(x), b(x), c(x))}$. By Tarski's Theorem, there is a semialgebraic set $T \subseteq \mathbb{R}^{n+m_{1}+m_{2}+m_{3}+n}$ such that for all $(x, a, b, c) \in \mathbb{R}^{n+m_{1}+m_{2}+m_{3}}$, we have $T_{(x, a, b, c)}=\operatorname{cl} S_{(x, a, b, c)}$. Therefore, the set

$$
C:=\{x \in U:(x, a(x), b(x), c(x), x) \in T\}
$$

is normal in $U$ and satisfies $\operatorname{fr}(A \cap V)=C \cap V$.

Combining Corollary 8.13 with Proposition 8.15, we obtain

Corollary 8.17. Let $m \leq n$. Then there is a finite collection $\mathcal{P}$ of normal leaflets in $U$ contained in $A$ such that

(i) $\Pi_{m}\left(\operatorname{cl}_{U}(A \cap V)\right)=\bigcup_{N \in \mathcal{P}} \Pi_{m}(N \cap V)$;

(ii) $\Pi_{m}(A \cap V)$ and $\Pi_{m}\left(\operatorname{fr}_{U}(A \cap V)\right)$ are unions of some of the $\Pi_{m}(N \cap V)$ with $N \in \mathcal{P}$;

(iii) for every $N \in \mathcal{P}$, the set $N \cap V$ is an analytic submanifold of $U$, the restriction of $\Pi_{m}$ to $N \cap V$ is an immersion, and for every $m^{\prime} \leq m$ the restriction of $\Pi_{m^{\prime}}$ to $N \cap V$ has constant rank. 


\section{Proper nested subpfaffian Sets}

In this section, we put $I:=[-1,1]$ and $I^{\prime}:=I \backslash\{0\}$ and let $m, n \geq 1$. To simplify terminology in this section, we write "pfaffian" in place of "pfaffian over $\mathcal{R} "$.

Definition 9.1. Let $Y \subseteq \mathbb{R}^{n}$ be closed, and assume there is a $U \subseteq \mathbb{R}^{n}$ such that $U$ is normal, $I^{n} \backslash Y \subseteq U$ and $U \cap Y=\emptyset$. Let $d=\left(d_{0}, \ldots, d_{k}\right)$ be a definable, analytic nested distribution on $U, V$ a Rolle leaf of $d$ and $A$ a normal subset of $U$. In this situation, we say that the basic nested pfaffian set $V \cap A \cap I^{n}$ is restricted off $Y$. A nested pfaffian set is restricted off $Y$ if it is a finite union of basic nested pfaffian sets that are restricted off $Y$.

Let $Z \subseteq \mathbb{R}^{m}$ be closed. A nested subpfaffian set $W \subseteq I^{m}$ is proper off $Z$ if $W$ is a finite union of sets of the form $\Pi_{m}^{n}(X)$, where $X \subseteq I^{n}$ is restricted nested pfaffian off $Z \times \mathbb{R}^{n-m}$.

Example 9.2. Let $X \subseteq I^{n}$ be restricted nested pfaffian off $\{0\}$. Then $X \backslash$ $\left(\{0\} \times \mathbb{R}^{n-1}\right)$ is restricted nested pfaffian off $\{0\} \times \mathbb{R}^{n-1}$.

Proposition 9.3. Let $W_{1}, \ldots, W_{q} \subseteq I^{m}$ be proper nested subpfaffian off $\{0\} \times \mathbb{R}^{m-1}$. Then there is a finite partition $\mathcal{C}$ of $I^{\prime} \times I^{m-1}$ into analytic cells compatible with $W_{1}, \ldots, W_{q}$ such that each $C \in \mathcal{C}$ is proper nested subpfaffian off $\{0\} \times \mathbb{R}^{m-1}$.

To prove Proposition 9.3, we first need to establish a few closure properties for the collection of all proper nested subpfaffian sets off $\{0\} \times \mathbb{R}^{m-1}$, for $m=1,2, \ldots$.

Lemma 9.4. The collection of all proper nested subpfaffian sets off $\{0\} \times$ $\mathbb{R}^{m-1}$, for $m=1,2, \ldots$, is closed under taking finite unions, projections on the first $k$ coordinates for $1 \leq k \leq m$, permutations of the last $m-1$ coordinates and topological closure inside $I^{m} \backslash\left(\{0\} \times \mathbb{R}^{m-1}\right)$.

Proof. Closure under taking finite unions, projections on the first $k$ coordinates with $k \in\{1, \ldots, m\}$ and permutations of the last $m-1$ coordinates is obvious from the definition. Closure with respect to taking topological closure inside $I^{m} \backslash\left(\{0\} \times \mathbb{R}^{m-1}\right)$ follows from Corollary 8.17 .

The collection of all proper nested subpfaffian sets off $\{0\} \times \mathbb{R}^{m-1}$, for $m=$ $1,2, \ldots$, is obviously not closed under taking cartesian products. However, we have the following weaker statement:

Lemma 9.5. Let $W \subseteq I^{m}$ be proper nested subpfaffian off $\{0\} \times \mathbb{R}^{m-1}$, and let $m^{\prime} \geq 1$ and $W^{\prime} \subseteq I^{m^{\prime}}$ be proper nested subpfaffian off $\{0\} \times \mathbb{R}^{m^{\prime}-1}$.

(1) $W \times I$ is proper nested subpfaffian off $\{0\} \times \mathbb{R}^{m}$. 
(2) Let $1 \leq k \leq \min \left\{m, m^{\prime}\right\}$. Write $(x, y)$ and $\left(x, y^{\prime}\right)$ for the elements of $\mathbb{R}^{m}$ and $\mathbb{R}^{m^{\prime}}$, respectively, where $x \in \mathbb{R}^{k}, y \in \mathbb{R}^{m-k}$ and $y^{\prime} \in \mathbb{R}^{m^{\prime}-k}$. Then the fiber product

$$
W \times_{k} W^{\prime}:=\left\{\left(x, y, y^{\prime}\right) \in \mathbb{R}^{m+m^{\prime}-k}:(x, y) \in W,\left(x, y^{\prime}\right) \in W^{\prime}\right\}
$$

is proper nested subpfaffian off $\{0\} \times \mathbb{R}^{m+m^{\prime}-k-1}$.

Sketch of proof. It suffices to consider the case where $W=\Pi_{m}(X)$ for some restricted nested pfaffian set $X \subseteq I^{n}$ off $\{0\} \times \mathbb{R}^{n-1}$.

(1) Arguing as in Corollary 3.4, we see that $W \times I=\Pi_{m+1}(Y)$, where

$$
Y:=\left\{(x, t, y) \in I^{n+1}: x \in I^{m}, y \in I^{n-m}, t \in I \text { and }(x, y) \in X\right\}
$$

is restricted nested pfaffian off $\{0\} \times \mathbb{R}^{n}$.

(2) We may also assume that $W^{\prime}=\Pi_{m^{\prime}}\left(X^{\prime}\right)$ for some restricted nested pfaffian set $X^{\prime} \subseteq I^{n^{\prime}}$ off $\{0\} \times \mathbb{R}^{n^{\prime}-1}$. Below, we let $z$ range over $I^{n-m}$ and $z^{\prime}$ range over $I^{n^{\prime}-m^{\prime}}$. Since $k \geq 1$, we have $W \times_{k} W^{\prime}=\Pi_{m+m^{\prime}-k}(Y)$, where

$$
Y:=\left\{\left(x, y, y^{\prime}, z, z^{\prime}\right) \in I^{n+n^{\prime}-k}:(x, y, z) \in X \text { and }\left(x, y^{\prime}, z^{\prime}\right) \in X^{\prime}\right\}
$$

is restricted nested pfaffian off $\{0\} \times \mathbb{R}^{n+n^{\prime}-k-1}$.

Corollary 9.6. Let $W, W^{\prime} \subseteq I^{m}$ be proper nested subpfaffian off $\{0\} \times \mathbb{R}^{m-1}$. Then $W \cap W^{\prime}$ is proper nested subpfaffian off $\{0\} \times \mathbb{R}^{m-1}$.

Proof. $W \cap W^{\prime}=W \times_{m} W^{\prime}$.

Also using Lemma 9.5, we obtain the following:

Lemma 9.7. Let $W_{1}, \ldots, W_{q} \subseteq I^{m}$ be proper nested subpfaffian off $\{0\} \times$ $\mathbb{R}^{m-1}$. Then

(1) the set

$W^{\prime}:=\left\{x^{\prime} \in I^{m-1}: \exists y_{1}<\cdots<y_{q},\left(x^{\prime}, y_{p}\right) \in W_{p}, p=1, \ldots, q\right\}$

is nested subpfaffian off $\{0\} \times \mathbb{R}^{m-2}$;

(2) for $p<q$ the set

$$
\begin{aligned}
W:=\left\{\left(x^{\prime}, y\right) \in I^{m}: \exists y_{1}<\cdots<y_{p}<y<y_{p+1}<\cdots<y_{q},\right. \\
\left.\quad\left(x^{\prime}, y_{l}\right) \in W_{l}, l=1, \ldots, q\right\}
\end{aligned}
$$

is nested subpfaffian off $\{0\} \times \mathbb{R}^{m-1}$;

(3) for $p \leq q$ the set

$$
\begin{aligned}
W:=\left\{\left(x^{\prime}, y\right) \in I^{m}: \exists y_{1}<\cdots<y_{p}=y<\cdots<y_{q},\right. \\
\left.\quad\left(x^{\prime}, y_{l}\right) \in W_{l}, l=1, \ldots, q\right\}
\end{aligned}
$$

is nested subpfaffian off $\{0\} \times \mathbb{R}^{m-1}$. 
Proof. We leave the details to the reader.

Proof of Proposition 9.3. By induction on $m$; the case $m=1$ follows from the o-minimality of $\mathcal{P}(\mathcal{R})$, so we assume that $m>1$ and the theorem holds for lower values of $m$. Increasing $q$ if necessary, we may assume that the singleton set $\{0\}$ and the sets $I^{m-1} \times\{-1\}$ and $I^{m-1} \times\{1\}$ are among the $W_{i}$. Decomposing each $W_{i}$ if necessary, we may also assume that for $p \in$ $\{1, \ldots, q\}$ there are $n_{p} \geq m$, a normal set $U_{p} \subseteq \mathbb{R}^{n_{p}} \backslash\left(\{0\} \times \mathbb{R}^{n_{p}-1}\right)$ containing $I^{n_{p}} \backslash\left(\{0\} \times I^{n_{p}-1}\right)$, a definable, analytic nested distribution $d^{p}=\left(d_{0}^{p}, \ldots, d_{k(p)}^{p}\right)$ on $U_{p}$, a Rolle leaf $V_{p}$ of $d^{p}$ and a normal subset $A_{p}$ of $U_{p}$ such that $W_{p}=$ $\Pi_{m}\left(A_{p} \cap V_{p}\right)$.

For $p \in\{1, \ldots, q\}$, we now apply Corollary 8.17 with $n_{p}, U_{p}, d^{p}, V_{p}$ and $A_{p}$ in place of $n, U, d, V$ and $A$. (Here we use the fact that the collection of all proper nested subpfaffian sets off $\{0\} \times \mathbb{R}^{m-1}$ is closed with respect to taking topological closure inside $I^{m} \backslash\left(\{0\} \times \mathbb{R}^{m-1}\right)$.) We let $\mathcal{P}_{p}$ be the corresponding collection of normal leaflets in $U_{p}$ obtained for $m$ and $\mathcal{P}_{p}^{\prime}$ be the corresponding collection of normal leaflets in $U_{p}$ obtained with $m-1$ in place of $m$, and we put

$$
\begin{gathered}
\mathcal{Q}:=\left\{\Pi_{m}\left(N \cap V_{p}\right): p \in\{1, \ldots, q\}, N \in \mathcal{P}_{p}, \operatorname{dim}\left(N \cap V_{p}\right)<m\right\}, \\
\mathcal{Q}^{\prime}:=\left\{\Pi_{m-1}\left(N \cap V_{p}\right): p \in\{1, \ldots, q\}, N \in \mathcal{P}_{p}^{\prime}, \operatorname{dim}\left(N \cap V_{p}\right)<m-1\right\} .
\end{gathered}
$$

By definition, the elements of $\mathcal{Q}$ and $\mathcal{Q}^{\prime}$ are proper nested subpfaffian sets off $\{0\} \times \mathbb{R}^{m-1}$ and $\{0\} \times \mathbb{R}^{m-2}$, respectively. Each $Z \in \mathcal{Q}$ is an immersed, analytic manifold in $\mathbb{R}^{m}$ with empty interior such that the restriction of $\Pi_{m-1}^{m}$ to $Z$ has constant rank. We put $\mathcal{Q}_{0}:=\left\{Z \in \mathcal{Q}:\left.\Pi_{m-1}\right|_{Z}\right.$ is an immersion $\}$ and let $F$ be the union of all sets in $\mathcal{Q}_{0}$. Similarly, each $Z^{\prime} \in \mathcal{Q}^{\prime}$ is an immersed, analytic manifold in $\mathbb{R}^{m-1}$ with empty interior.

Since every $Z \in \mathcal{Q}$ is definable in $\mathcal{P}(\mathcal{R})$, there exists an $N \in \mathbb{N}$ such that $Z_{x^{\prime}}$ has at most $N$ components for every $x^{\prime} \in \mathbb{R}^{m-1}$ and every $Z \in \mathcal{Q}$. For $k \leq N|\mathcal{Q}|$ and $Z_{1}, \ldots, Z_{k} \in \mathcal{Q}$, we put

$$
Z^{\prime}\left(Z_{1}, \ldots, Z_{k}\right):=\left\{x^{\prime} \in I^{m-1}: \exists y_{1}<\cdots<y_{k}, \quad\left(x^{\prime}, y_{j}\right) \in Z_{j} \text { for each } j\right\},
$$

and we denote by $\mathcal{Q}^{\prime \prime}$ the collection of these sets. (Note in particular that the projections on the first $m-1$ coordinates of all $Z \in \mathcal{Q}$ belong to $\mathcal{Q}^{\prime \prime}$.) By Lemma 9.7, each set in $\mathcal{Q}^{\prime \prime}$ is proper nested subpfaffian off $\{0\} \times \mathbb{R}^{m-2}$. Hence by the inductive hypothesis applied to the collection $\mathcal{Q}^{\prime} \cup \mathcal{Q}^{\prime \prime}$, there is a finite partition $\mathcal{C}^{\prime}$ of $I^{\prime} \times I^{m-2}$ into analytic cells such that $\mathcal{C}^{\prime}$ is compatible with $\mathcal{Q}^{\prime} \cup \mathcal{Q}^{\prime \prime}$ and each $C^{\prime} \in \mathcal{C}^{\prime}$ is proper nested subpfaffian off $\{0\} \times \mathbb{R}^{m-2}$.

We now fix $C^{\prime} \in \mathcal{C}^{\prime}$; it suffices to show that $C^{\prime} \times I$ admits a finite partition $\mathcal{C}$ into analytic cells such that $\mathcal{C}$ is compatible with $\left\{W_{1}, \ldots, W_{q}\right\}$ and each $C \in \mathcal{C}$ is proper nested subpfaffian off $\{0\} \times \mathbb{R}^{m-2}$. However, for $p \in\{1, \ldots, q\}$, the set $W_{p} \cap\left(C^{\prime} \times I\right)$ is the union of some of the sets $Z \cap\left(C^{\prime} \times I\right)$ with $Z \in \mathcal{Q}_{0}$ 
and some of the components of $\left(C^{\prime} \times I\right) \backslash F$. Therefore, it suffices to show that $C^{\prime} \times I$ admits a finite partition $\mathcal{C}$ into analytic cells such that $\mathcal{C}$ is compatible with $\mathcal{Q}_{0}$ and each $C \in \mathcal{C}$ is proper nested subpfaffian off $\{0\} \times \mathbb{R}^{m-2}$.

By construction, Lemma 9.5 and Corollary 9.6, if $Z \in \mathcal{Q}_{0}$ then the set $Z \cap\left(C^{\prime} \times I\right)$ is proper nested subpfaffian off $\{0\} \times \mathbb{R}^{m-1}$ and an analytic submanifold of $I^{\prime} \times I^{m-1}$, and each of its components is the graph of an analytic function from $C^{\prime}$ to $\mathbb{R}$. In particular, $F \cap\left(C^{\prime} \times I\right)$ is a closed subset of $C^{\prime} \times I$. Moreover, if $Y \in \mathcal{Q}_{0}$ also, then $(Z \cap Y) \cap\left(C^{\prime} \times I\right)$ is the union of some of the components of $Z \cap\left(C^{\prime} \times I\right)$. On the other hand, each component of $Z \cap\left(C^{\prime} \times I\right)$ is of the form

$$
\left\{\left(x^{\prime}, y\right) \in C^{\prime} \times I: \exists y_{1}<\cdots<y_{k}, y=y_{l} \text { and }\left(x^{\prime}, y_{j}\right) \in Z_{j} \text { for each } j\right\},
$$

where $k \leq N|\mathcal{Q}|, l \leq k$ and $Z_{1}, \ldots, Z_{k} \in \mathcal{Q}$. Hence by Lemma 9.7, each such component is proper nested subpfaffian off $\{0\} \times \mathbb{R}^{m-1}$ and an analytic cell. It follows that each component of $\left(C^{\prime} \times I\right) \backslash F$ is an open analytic cell, and each such component is proper nested subpfaffian off $\{0\} \times \mathbb{R}^{m-1}$ by Lemma 9.7 again, because it is of the form

$\left\{\left(x^{\prime}, y\right) \in C^{\prime} \times I: \exists y_{1}<\cdots<y_{k}, y_{l}<y<y_{l+1}\right.$ and $\left(x^{\prime}, y_{j}\right) \in Z_{j}$ for each $\left.j\right\}$ with $k \leq N|\mathcal{Q}|, l<k$ and $Z_{1}, \ldots, Z_{k} \in \mathcal{Q}$.

\section{REWRITING NESTED INTEGRAL MANIFOLDS}

Assume that $\mathcal{R}$ admits analytic cell decomposition. Let $M \subseteq \mathbb{R}^{n}$ be an analytic, definable manifold, and let $d=\left(d_{0}, \ldots, d_{k}\right)$ be an analytic, definable nested distribution on $M$. Let also $A \subseteq M$ be definable.

Proposition 10.1. There are $n_{1}, \ldots, n_{s} \in \mathbb{N}$ and, for $j=1, \ldots, s$, there exist an analytic, definable nested distribution $e_{j}$ on $C_{j}:=\left\{y \in \mathbb{R}^{n_{j}}: 0<|y|<2\right\}$ and a definable, analytic embedding $\psi_{j}: C_{j} \longrightarrow M$ such that, with $B_{j}:=$ $\left\{y \in \mathbb{R}^{n_{j}}: 0<|y|<1\right\}$,

(i) $\psi_{j}\left(C_{j}\right) \subseteq A$ for each $j$ and the collection $\left\{\psi_{j}\left(B_{j}\right): j=1, \ldots, s\right\}$ covers A;

(ii) for every Rolle leaf $V$ of $d$, we have $A \cap V=\bigcup_{j=1}^{s} \psi_{j}\left(B_{j} \cap V_{j}\right)$, where each $V_{j}$ is either empty or a Rolle leaf of $e_{j}$.

Remark. Each $B_{j} \cap V_{j}$ is a restricted nested pfaffian set off $\{0\}$.

Proof. By Corollary [3.5, we may assume that $A=M=\mathbb{R}^{n}$. If $d$ has no Rolle leaves, the proposition is now trivial. So we also assume that $d$ has a Rolle leaf; in particular, $d_{1}$ has a Rolle leaf $V_{1}$, say. Then $V_{1}$ is embedded, closed and of codimension 1 in $\mathbb{R}^{n}$, so $V_{1}$ separates $\mathbb{R}^{n}$. Let $D_{1}$ and $D_{2}$ be closed boxes in $\mathbb{R}^{n} \backslash V_{1}$ with nonempty interior and contained in different components of $\mathbb{R}^{n} \backslash V_{1}$, and denote by $c_{1}$ and $c_{2}$ their centers and by $U_{1}$ and $U_{2}$ their complements 
in $\mathbb{R}^{n}$. For $j=1,2$, let $D_{j}^{\prime}$ be a closed box with center $c_{j}$ such that $D_{j}^{\prime}$ is contained in the interior of $D_{j}$, and put $U_{j}^{\prime}:=\mathbb{R}^{n} \backslash D_{j}^{\prime}$. Let $\phi_{j}: \mathbb{R}^{n} \backslash\left\{c_{j}\right\} \longrightarrow$ $\mathbb{R}^{n} \backslash\{0\}$ be a definable, analytic diffeomorphism such that $\phi_{j}\left(U_{j}^{\prime}\right)=C:=$ $\left\{x \in \mathbb{R}^{n}: 0<|x|<2\right\}$ and $\phi_{j}\left(U_{j}\right)=B:=\left\{x \in \mathbb{R}^{n}: 0<|x|<1\right\}$. We let $e_{j}$ be the push-forward of the restriction of $d$ to $\mathbb{R}^{n} \backslash\left\{c_{j}\right\}$ via $\phi_{j}$ and put $\psi_{j}:=\phi_{j}^{-1}$. Note that $V_{1} \subseteq \psi_{j}(B)$ for each $j$, and each component of $\mathbb{R}^{n} \backslash V_{1}$ is contained in $\psi_{j}(B)$ for one $j$. Therefore, any Rolle leaf of $d$ is contained in $\psi_{j}(B)$ for at least one $j$. The corollary now follows.

Corollary 10.2. Let $V$ be a Rolle leaf of $d$. Then $A \cap V$ is a finite union of simply connected nested subpfaffian sets over $\mathcal{R}$ that are analytic manifolds.

Proof. By induction on $\delta:=\operatorname{dim} A$; if $\delta=0$, there is nothing to do, so we assume $\delta>0$ and the corollary holds for lower values of $\delta$. By the previous proposition and the inductive hypothesis, we may assume that $M=\left\{x \in \mathbb{R}^{\delta}\right.$ : $0<|x|<2\}$ and $A=\left\{x \in \mathbb{R}^{\delta}: 0<|x|<1\right\}$, and we let $V$ be a Rolle leaf of $d$. Since $A \cap V$ is restricted nested pfaffian off $\{0\}$, the corollary now follows with $(A \cap V) \backslash\left(\{0\} \times \mathbb{R}^{\delta-1}\right)$ in place of $A \cap V$ from Example 9.2 and Proposition 9.3. Since $A \cap\left(\{0\} \times \mathbb{R}^{\delta-1}\right)$ has dimension $\delta-1$, the corollary with $A \cap V \cap\left(\{0\} \times \mathbb{R}^{\delta-1}\right)$ in place of $A \cap V$ follows from the inductive hypothesis.

For Lemma 10.3 and Proposition 10.4 below, we let $\mathcal{S}$ be an o-minimal expansion of $\mathcal{R}$.

Lemma 10.3. Let $\left(W_{0}, \ldots, W_{k}\right)$ be a nested integral manifold of definable in $\mathcal{S}$ and $\nu \leq n$, and assume that $d$ is analytic and integrable and $\left(W_{0}, \ldots, W_{k-1}\right)$ is a nested Rolle leaf over $\mathcal{R}$. Then there is a nested subpfaffian set $Y \subseteq \mathbb{R}^{n}$ such that $W_{k} \subseteq Y$ and $\operatorname{dim} \Pi_{\nu}(Y) \leq \operatorname{dim} \Pi_{\nu}\left(W_{k}\right)$.

Proof. By induction on $\operatorname{dim} W_{k}$; if $\operatorname{dim} W_{k}=0$, there is nothing to do, so we assume $\operatorname{dim} W_{k}>0$ and the lemma holds for lower values of $\operatorname{dim} W_{k}$. By Corollary 10.2, there are simply connected nested subpfaffian sets $Y_{1}, \ldots, Y_{q} \subseteq$ $\mathbb{R}^{n}$ such that each $Y_{p}$ is an analytic submanifold of $\mathbb{R}^{n}$ and $W_{k-1}=Y_{1} \cup \cdots \cup$ $Y_{q}$. By Corollary 8.14, for each $p$, there are $n_{p} \geq n$ and nested pfaffian sets $Z_{p, 1}, \ldots, Z_{p, k(p)} \subseteq \mathbb{R}^{n_{p}}$ over $\mathcal{R}$ such that $Y_{p}=\Pi_{n}\left(Z_{p, 1}\right) \cup \cdots \cup \Pi_{n}\left(Z_{p, k(p)}\right)$ and each $Z_{p, j}$ is an analytic manifold, $\Pi_{n}\left\lceil z_{p, j}\right.$ is an immersion and $\Pi_{\nu} \Upsilon_{p, j}$ has constant rank. Refining the $Z_{p, j}$ further if necessary and using Corollary [3.3(1), we may assume in addition that each $Z_{p, j}$ is a Rolle leaf over $\mathcal{R}$ obtained from a definable, analytic, integrable nested distribution $d_{p, j}=\left(d_{p, j, 0}, \ldots, d_{p, j, k(p, j)}\right)$ on a definable analytic manifold $M_{p, j} \subseteq \mathbb{R}^{n_{p}}$ such that $\Pi_{n}\left(M_{p, j}\right) \subseteq M$, and that the dimension of the spaces $F_{p, j}(y):=\Pi_{n}\left(d_{p, j, k(p, j)}(y)\right)$ and $E_{p, j}(y):=$ $F_{p, j}(y) \cap d_{k}\left(\Pi_{n}(y)\right)$ and of their projections $\Pi_{\nu}\left(F_{p, j}(y)\right)$ and $\Pi_{\nu}\left(E_{p, j}(y)\right)$ are constant as $y$ ranges over $M_{p, j}$. Note in particular that $F_{p, j}(y) \subseteq d_{k-1}\left(\Pi_{n}(y)\right)$ 
and $\operatorname{dim} F_{p, j}(y)=\operatorname{dim} d_{p, j, k(p, j)}(y)$ for $y \in M_{p, j}$; we denote by $\Lambda$ the set of all pairs $(p, j)$ such that $F_{p, j}(y) \neq E_{p, j}(y)$ for $y \in M_{p, j}$.

For $(p, j) \in \Lambda$ and $y \in M_{p, j}$, we now define $d_{p, j, k(p, j)+1}(y):=d_{p, j, k(p, j)}(y) \cap$ $\Pi_{n}^{-1}\left(E_{p, j}(y)\right)$. Then $d_{p, j}^{\prime}:=\left(d_{p, j, 0}, \ldots, d_{p, j, k(p, j)+1}\right)$ is a definable nested distribution on $M_{p, j}$, and the set $Z_{p, j}^{\prime}:=\Pi_{n}^{-1}\left(W_{k}\right) \cap Z_{p, j}$ is an integral manifold of $d_{p, j}^{\prime}$ definable in $\mathcal{S}$. Similarly, for $(p, j) \notin \Lambda$, the set $Z_{p, j}^{\prime}:=\Pi_{n}^{-1}\left(W_{k}\right) \cap Z_{p, j}$ is an integral manifold of $d_{p, j}^{\prime}:=d_{p, j}$ definable in $\mathcal{S}$.

We now fix $(p, j)$; it suffices to prove the lemma with $Z_{p, j}^{\prime}$ in place of $W_{k}$. If $(p, j) \notin \Lambda$, we conclude with $Y:=Z_{p, j}$; so we assume from now on that $(p, j) \in \Lambda$. If $F_{p, j}(y) \neq d_{k-1}\left(\Pi_{n}(y)\right)$ for $y \in M_{p, j}$, then $\operatorname{dim} Z_{p, j}^{\prime}<\operatorname{dim} W_{k}$ and we conclude using the inductive hypothesis; so we assume also from now on that $F_{p, j}(y)=d_{k-1}\left(\Pi_{n}(y)\right)$ for $y \in M_{p, j}$. Let $L$ be a leaf of $d_{p, j}^{\prime}$ such that $L \cap Z_{p, j}^{\prime} \neq \emptyset$; then $L \subseteq Z_{p, j}$. We claim that $L$ is a Rolle leaf of $d_{p, j}^{\prime}$; this claim finishes the proof of the lemma, because $\operatorname{dim} \Pi_{\nu}(L) \leq \operatorname{dim} \Pi_{\nu}\left(W_{k}\right)$ by construction. To see the claim, note that $Y_{p}$ is an open subset of $W_{k-1}$ and $\Pi_{n}(L)$ is a connected integral manifold of $d_{k}^{Y_{p}}$. By Haefliger's Theorem, the leaf $L^{\prime}$ of $d_{k}^{Y_{p}}$ containing $\Pi_{n}(L)$ is a Rolle leaf. On the other hand, since $Z_{p, j}$ is a Rolle leaf and $\Pi_{n} \uparrow_{d_{p, j, k(p, j)}(y)}$ is an immersion for $y \in M_{p, j}$, the set $Z_{p, j}$ is the graph of a function $g_{p, j}: \Pi_{n}\left(Z_{p, j}\right) \longrightarrow \mathbb{R}^{n_{p}-n}$. Thus, if $\gamma:[0,1] \longrightarrow Z_{p, j}$ is a curve intersecting $L$ in two distinct points, then $\Pi_{n} \circ \gamma:[0,1] \longrightarrow Y_{p}$ is a curve intersecting $L^{\prime}$ in two distinct points, so $\Pi_{n} \circ \gamma$ is tangent to $d_{k}$ at some point. It follows that $\gamma$ is tangent to $d_{p, j, k(p, j)+1}$ at some point, as required.

Proposition 10.4. Let $W \subseteq \mathbb{R}^{n}$ be an integral manifold over $\mathcal{R}$ definable in $\mathcal{S}$ and $\nu \leq n$. Then there is a nested subpfaffian set $Y \subseteq \mathbb{R}^{n}$ such that $W \subseteq Y$ and $\operatorname{dim} \Pi_{\nu}(Y) \leq \operatorname{dim} \Pi_{\nu}(W)$.

Proof. We proceed by induction on $k$. If $k=0$ there is nothing to do, so we assume $k>0$ and the proposition holds for lower values of $k$. By the inductive hypothesis and Corollary 3.3. (1), there exists a $q \in \mathbb{N}$, and for $p=1, \ldots, q$ there exist $n_{p} \geq n$, a definable manifold $N_{p} \subseteq \mathbb{R}^{n_{p}}$, a definable nested distribution $f_{p}=\left(f_{p, 0}, \ldots, f_{p, k(p)}\right)$ on $N_{p}$ and a nested Rolle leaf $V_{p}=\left(V_{p, 0}, \ldots, V_{p, k(p)}\right)$ of $f_{p}$ such that $W_{k-1} \subseteq \bigcup_{p=1}^{q} \Pi_{n}\left(V_{p, k(p)}\right)$ and $\operatorname{dim} \Pi_{\nu}\left(V_{p, k(p)}\right) \leq \operatorname{dim} \Pi_{\nu}\left(W_{k-1}\right)$ for each $p$. After refining and pruning the collection $\left\{N_{1}, \ldots, N_{q}\right\}$ if necessary, we may assume that that

$(\dagger) \Pi_{n}\left(N_{p}\right) \subseteq M$ and $\Pi_{n}\left(V_{p, k(p)}\right) \cap W_{k} \neq \emptyset$ for each $p$, and that $W_{k} \subseteq$ $\Pi_{n}\left(V_{1, k(1)}\right) \cup \cdots \cup \Pi_{n}\left(V_{q, k(q)}\right)$.

We now finish the proof of the proposition by induction on

$$
\delta:=\max \left\{\operatorname{dim} \Pi_{\nu}\left(V_{p, k(p)}\right): p=1, \ldots, q\right\}
$$


simultaneously for all such collections $\left\{\left(N_{p}, f_{p}, Z_{p}\right)\right\}$ satisfying $(\dagger)$. If $\delta=$ $\operatorname{dim} \Pi_{\nu}\left(W_{k}\right)$, we are now done. So assume $\delta>\operatorname{dim} \Pi_{\nu}\left(W_{k}\right)$ and the proposition holds for lower values of $\delta$. Refining and pruning the collection $\left\{N_{1}, \ldots, N_{q}\right\}$ again if necessary, we may assume for each $p$, each $\mu \leq \nu$ and each strictly increasing $\lambda:\{1, \ldots, \mu\} \longrightarrow\{1, \ldots, \nu\}$ that the dimension of the spaces

$$
\Pi_{\lambda}\left(f_{p, k(p)}(y)\right) \text { and } \Pi_{\lambda}\left(\Pi_{\nu}\left(f_{p, k(p)}(y)\right) \cap \Pi_{\nu}\left(d_{k}\left(\Pi_{n}(y)\right)\right)\right)
$$

is constant as $y$ ranges over $N_{p}$. Then by Proposition 6.3 and Lemma 10.3 , for each $p$ such that $\operatorname{dim} \Pi_{\nu}\left(V_{p, k(p)}\right)=\delta$, the Rolle leaf $V_{p, k(p)}$ over $\mathcal{R}$ can be replaced by finitely many Rolle leaves $V^{\prime}$ over $\mathcal{R}$ such that $\operatorname{dim} \Pi_{\nu}\left(V^{\prime}\right)<\delta$. The proposition now follows from the inductive hypothesis.

Proofs of Proposition 1 and Theorem 1. Let $W \subseteq \mathbb{R}^{n}$ be a Rolle leaf over $\mathcal{R}$; we proceed by induction on $\operatorname{dim} W$. If $\operatorname{dim} W=0$, there is nothing to do, so we assume $\operatorname{dim} W>0$ and Theorem 1 holds for lower values of $\operatorname{dim} W$. Using the definable, analytic diffeomorphism $t \mapsto t / \sqrt{1+t^{2}}: \mathbb{R} \longrightarrow(-1,1)$ in each coordinate, we may assume that $M$ is bounded. By analytic cell decomposition and Remark 4.9, we may assume that $M$ is an analytic cell. By Proposition 4.6, there are pfaffian limits $K_{1}, \ldots, K_{q} \subseteq \mathbb{R}^{n}$ over $\mathcal{R}$ such that fr $W \subseteq K_{1} \cup \cdots \cup K_{q}$ and $\operatorname{dim} K_{p}<\operatorname{dim} W$ for each $p$. By Proposition 7.1, we may assume for each $p$ that $K_{p}=\Pi_{n}\left(U_{p}\right)$ for some integral manifold $U_{p} \subseteq \mathbb{R}^{n_{p}}$ over $\mathcal{R}$ definable in $\mathcal{P}(\mathcal{R})$ such that $n_{p} \geq n$ (this proves Proposition 1 ). By Proposition 10.4 with $U_{p}$ in place of $W$, we may assume that each $K_{p}$ is nested subpfaffian over $\mathcal{R}$, say $K_{p}=\Pi_{n}\left(Y_{p}\right)$ for some nested pfaffian set $Y_{p} \subseteq \mathbb{R}^{m_{p}}$ over $\mathcal{R}$ such that $m_{p} \geq n_{p}$. By Corollaries [3.3(1) and 8.14, we may assume that each $Y_{p}$ is a Rolle leaf over $\mathcal{R}$ such that $\Pi_{n} \uparrow_{Y_{p}}$ is an immersion; in particular, $\operatorname{dim} Y_{p}<\operatorname{dim} W$. Hence by the inductive hypothesis, there is a closed nested subpfaffian set $Z_{p} \subseteq \mathbb{R}^{m_{p}}$, for each $p$, such that fr $Y_{p} \subseteq Z_{p}$ and $\operatorname{dim} Z_{p}<\operatorname{dim} W$. Then the union of the sets $\Pi_{n}\left(Y_{p} \cup Z_{p}\right)$, for $p=1, \ldots, q$, is a closed nested subpfaffian set containing fr $W$ and of dimension strictly less than $\operatorname{dim} W$, as required.

\section{Conclusion}

We conclude by proving the corollary in the introduction; we continue to assume that $\mathcal{R}$ admits analytic cell decomposition. Let $L \subseteq \mathbb{R}^{n}$ be one of the Rolle leaves added to $\mathcal{R}$ in the construction of $\mathcal{P}(\mathcal{R})$ in [20]; it suffices to establish the following:

Proposition 11.1. $L$ is a nested subpfaffian set over $\mathcal{R}$.

Proof. By construction of $\mathcal{P}(\mathcal{R})$ and Example 1, there are an $l \in \mathbb{N}$ and an $(n-1)$-distribution $e$ on $\mathbb{R}^{n}$ definable in $\mathcal{R}_{l}$ such that $L$ is a Rolle leaf of $e$. We proceed by induction on $l$; if $l=0$, we are done, so we assume that $l>0$ and the 
proposition holds for lower values of $l$; in particular, every set definable in $\mathcal{R}_{l}$ is definable in $\mathcal{N}(\mathcal{R})$. Thus, by the Main Theorem, analytic cell decomposition and Corollary 3.3.(1), we may assume that there are $n^{\prime} \geq n+n^{2}$, a definable, analytic manifold $M \subseteq \mathbb{R}^{n^{\prime}}$, a definable, analytic nested distribution $d=$ $\left(d_{0}, \ldots, d_{k}\right)$ on $M$ and a Rolle leaf $V$ of $d$ such that gr $e=\Pi_{n+n^{2}}(V)$. By Corollary 8.14, we may further assume that $\Pi_{n+n^{2}} \Upsilon_{V}$ is an immersion and $\operatorname{dim} \Pi_{n+n^{2}}\left(d_{k}(y)\right)=\operatorname{dim} d_{k}(y)=n$ for all $y \in M$. Since $\Pi_{n+n^{2}}(V) \subseteq \mathbb{R}^{n} \times G_{n}^{n-1}$, we may also assume that $\Pi_{n+n^{2}}(M) \subseteq \mathbb{R}^{n} \times G_{n}^{n-1}$. Let now $d_{k+1}$ be the $(n-1)$ distribution on $M$ defined by

$$
d_{k+1}(y):=d_{k}(y) \cap\left(\Pi_{n}^{n^{\prime}}\right)^{-1}\left(\pi\left(\Pi_{n+n^{2}}(y)\right)\right),
$$

where $\pi: \mathbb{R}^{n+n^{2}} \longrightarrow \mathbb{R}^{n^{2}}$ is the projection on the last $n^{2}$ coordinates and $\pi\left(\Pi_{n+n^{2}}(y)\right)$ is identified with the $(n-1)$-dimensional subspace of $\mathbb{R}^{n}$ that it represents. Then $d^{\prime}:=\left(d_{0}, \ldots, d_{k+1}\right)$ is a definable nested distribution on $M$ and $V^{\prime}:=V \cap\left(\Pi_{n}^{n^{\prime}}\right)^{-1}(L)$ is a Rolle leaf of $d_{k+1}$, as required.

\section{REFERENCES}

[1] J. Bochnak, M. Coste, And F. Roy, Real Algebraic Geometry, Springer Verlag, 1998.

[2] C. Camacho and A. Lins Neto, Geometric theory of foliations, Birkhäuser, 1985.

[3] F. CAno, J.-M. Lion, And R. Moussu, Frontière d'une hypersurface pfaffienne, Ann. Sci. Ecole Norm. Sup. (4), 28 (1995), pp. 591-646.

[4] L. VAN DEN DRIES, Limit sets in o-minimal structures, Proceedings of the RAAG Summer School Lisbon 2003: O-minimal structures, Eds M. Edmundo, D. Richardson, A.J. Wilkie (2005), pp. 172-215.

[5] L. VAn den Dries AND C. Miller, Geometric categories and o-minimal structures, Duke Math. J., 84 (1996), pp. 497-540.

[6] L. VAN DEN Dries AND P. SpeissegGer, The real field with convergent generalized power series is model complete and o-minimal, Trans. Amer. Math. Soc., 350 (1998), pp. $4377-4421$.

[7] A. Gabrielov, Multiplicities of Pfaffian intersections and the Eojasiewicz inequality, Sel. math., New Series, 1 (1995), pp. 113-127.

[8] - Complements of subanalytic sets and existential formulas for analytic functions, Invent. Math., 125 (1996), pp. 1-12.

[9] — Frontier and closure of a semi-pfaffian set, J. Discrete and Computational Geometry, 19 (1998), pp. 605-617.

[10] A. Haefliger, Structures feuilletées et cohomologie à valeurs dans un faisceau de groupoïdes, Comment. Math. Helv., 32 (1958), pp. 248-329.

[11] A. KhovanskiI, Fewnomials, vol. 88 of Translations of Mathematical Monographs, American Mathematical Society, 1991.

[12] K. Kuratowski, Topology, Academic Press, 1966.

[13] J.-M. Lion And J.-P. Rolin, Volumes, feuilles de Rolle de feuilletages analytiques réelles et théorème de Wilkie, Ann. Toulouse, 7 (1998), pp. 93-112.

[14] J.-M. Lion And P. Speissegger, A geometric proof of the definability of Hausdorff limits, Sel. math., New series. 10 (2004), pp. 377-390. 
[15] — Analytic stratification in the Pfaffian closure of an o-minimal structure, Duke Math. J., 103 (2000), pp. 215-231.

[16] —, Un théorème de type Haefliger définissable, Differential Equations and Singularities: 60 years of J. M. Aroca, Ed F. Cano Torres, Astérisque, 323 (2009).

[17] D. Marker and C. Steinhorn, Definable types in o-minimal theories, J. Symbolic Logic, 59 (1994), pp. 185-441.

[18] R. Moussu And C. Roche, Théorie de Khovanskii et problème de Dulac, Invent. Math., 105 (1991), pp. 431-441.

[19] — Théorèmes de finitude uniformes pour les variétés Pfaffiennes, Ann. Inst. Fourier, 42 (1992), pp. 393-420.

[20] P. Speissegger, The Pfaffian closure of an o-minimal structure, J. Reine Angew. Math., 508 (1999), pp. 189-211.

[21] A. J. WILKIE, Model completeness results for expansions of the ordered field of real numbers by restricted Pfaffian functions and the exponential function, J. Amer. Math. Soc., 9 (1996), pp. 1051-1094.

[22] — A theorem of the complement and some new o-minimal structures, Sel. math., 5 (1999), pp. 397-421.

[23] — Covering definable open sets by open cells, Proceedings of the RAAG Summer School Lisbon 2003: O-minimal structures, Eds M. Edmundo, D. Richardson, A.J. Wilkie (2005), pp. 32-36.

UFR mathématiques, Université de Rennes I, Campus de Beaulieu, 35042 RENNES CEDEX, FRANCE

E-mail address: jean-marie.lion@univ-rennes1.fr

Department of Mathematics \& Statistics, McMaster University, 1280 Main Street West, Hamilton, Ontario L8S 4K1, Canada

E-mail address: speisseg@math.mcmaster.ca 